This item was submitted to Loughborough's Research Repository by the author.

Items in Figshare are protected by copyright, with all rights reserved, unless otherwise indicated.

\title{
Strategies for the replacement of chromic acid anodising for the structural bonding of aluminium alloys
}

PLEASE CITE THE PUBLISHED VERSION

PUBLISHER

(C) Elsevier

LICENCE

CC BY-NC-ND 4.0

REPOSITORY RECORD

Critchlow, Gary W., Keith A. Yendall, D. Bahrani, A. Quinn, and F. Andrews. 2006. "Strategies for the Replacement of Chromic Acid Anodising for the Structural Bonding of Aluminium Alloys". figshare. https://hdl.handle.net/2134/807. 


\title{
STRATEGIES FOR THE REPLACEMENT OF CHROMIC ACID ANODISING FOR THE STRUCTURAL BONDING OF ALUMINIUM ALLOYS
}

\author{
G.W.Critchlow $^{1 *}$, K.A.Yendall ${ }^{1}$, D.Bahrani ${ }^{2}$, A.Quinn ${ }^{2}$ and F.Andrews ${ }^{3}$ \\ ${ }^{1}$ Institute of Surface Science \& Technology, IPTME, Loughborough University, \\ Loughborough, Leicestershire, LE11 3TU, UK. \\ ${ }^{2}$ Short Bros./Bombardier Aerospace, Airport Road, Belfast, BT3 9DZ, Northern \\ Ireland, UK. \\ ${ }^{3}$ Thales Air Defence, Alanbrooke Road, Castlereagh, Belfast, Northern Ireland, UK \\ *corresponding author, g.w.critchlow@lboro.ac.uk
}

\begin{abstract}
The 40/50V Bengough-Stuart chromic acid anodise process is widely used in demanding applications as a prebond treatment. This process has a number of disadvantages and its replacement is the subject of much interest in the aerospace, automotive and defence sectors, amongst others. This paper details a number of modifications to the standard boric-sulphuric acid anodising (BSAA) process specifically to achieve satisfactory structural bond performance. These included: variations in the deoxidiser and anodising parameters, and; the use of a post anodising dip. It has been demonstrated in these studies that there are three possible methods of providing excellent durability using a variation of the standard BSAA process: the use of an electrolytic phosphoric acid deoxidiser (EPAD); a high temperature anodise at $35^{\circ} \mathrm{C}$, and; the use of a post anodise phosphoric acid dip (PAD).
\end{abstract}


Adhesive bonding of aluminium and its alloys is utilised in many manufacturing sectors. The majority of studies in the literature derive from the aerospace, defence or automotive sectors or their materials suppliers ${ }^{1-6}$. However, the use of bonded aluminium in other sectors such as the rail, shipbuilding, general engineering or construction industries should not be overlooked. Reflecting this widespread usage, the requirements for a bonded aluminium assembly may vary widely. In simple terms, usage may be described as being, structural, secondary or non-structural. The present study is concerned with replacement of chromic acid anodising (CAA) a prebond treatment process used in structural or secondary applications, particularly within the aerospace sector. Structural applications are defined as those in which a bonded assembly is expected to maintain a significant load for most of its service life; secondary applications include those in which the bond is complemented by a further joining technique such as welds or rivets. In either case, the load may be static, cyclic or intermittent and may be combined with exposure to harsh environmental conditions. Aerospace components are clearly exposed to such conditions. It should be noted that although the bonding of aerospace alloys is the focus of this study, the processes described are applicable to the majority of aluminium alloys.

The advantages that adhesive bonding offers can be put in to context by considering a number of alternative joining methods. Firstly, it is worth mentioning at this stage that riveting is the most established form of joining for aircraft assemblies ${ }^{7}$. A disadvantage to this joining method is that the fasteners produce points of high stress concentration, when compared to bonded joints, which could lead to premature failure particularly in fatigue conditions ${ }^{1,2,8}$. Secondary, hybrid riv-bonded or weld-bonded assemblies are constructed to provide some of the advantages of both joining methods ${ }^{9-11}$. Also it has been reported that welding can solve many of the problems associated with riveting as a means of jointing aerospace materials. However, there have been claims that the $2 \mathrm{xxx}$ and $7 \mathrm{xxx}$ aerospace alloys are 'unweldable' as these heat-treated alloys can suffer from solidification cracking ${ }^{12}$. Recent work, however, claims successful weldability of these alloys with additions of scandium to the basic alloy composition. Scandium is reported to induce a very fine grain size in the weld microstructure ${ }^{13}$. The increasing drive to be able to weld aerospace alloys has 
encouraged developments in specialised TIG and MIG applications for welding the $2 \mathrm{xxx}$ and $7 \mathrm{xxx}$ alloys ${ }^{13}$. Laser welding has also been developed using both $\mathrm{CO}_{2}$ and Nd:YAG sources. These are rapid, flexible and posses relatively low distortion properties $^{13}$. Friction welding is another area of research ${ }^{14}$ with conventional rotary friction welding, linear friction welding and friction stir welding. Of these, the friction stir welding has shown to be most successful with $2 \mathrm{xxx}, 6 \mathrm{xxx}$, and $7 \mathrm{xxx}$ alloys giving good tensile, bend and fatigue properties ${ }^{13}$. However, there are concerns for friction stir welding of 7075-T6 alloys and its susceptibility to inter-granular attack, especially at the hottest regions of the weld zones ${ }^{14}$.

However, adhesively bonded joints are reportedly superior over mechanically fastened joints in a number of ways, by providing: high strength to weight ratios; increased fatigue life; simplified design; smooth external finish, and; the ability to join dissimilar materials. It should also be noted that there are concerns regarding the use of adhesive bonding including: the lack of confidence in durability; the lack of proven non-destructive inspection methods, and; the limited high temperature performance. All of these areas are the focus of ongoing research ${ }^{15-20}$.

In order to achieve both good initial adhesion and durability the entire bonded system must be considered ie. the alloy-pretreatment-adhesive/primer combination. In the present study we are not concerned with the influence of varying the adhesive/primer combination. The pretreatment or surface engineering of various aluminium alloys is, however, discussed. The study of the influence of prebond treatments or pretreatment of aluminium is also a significant area of research; substantial data on this topic have been assembled, particularly by Minford ${ }^{1}$ and to a lesser extent by Critchlow and Brewis $^{21}$. A brief summary of the literature follows.

\subsection{Surface pretreatments for adhesive bonding}

Ideally, a particular pretreatment for structural bonding of aluminium will produce a surface which is: free from contamination; wettable by the adhesive; highly macro- or micro-rough; mechanically stable, and; hydrolytically stable. 
With metallic adherends the first two issues are intimately related as atomically-clean metal or metal oxides, being high energy surfaces, will be easily wettable by a subsequently applied primer or adhesive ${ }^{22}$. Aluminium oxide has a high surface energy with values reportedly of many hundreds of millijoules per square metre. This is more than adequate for an epoxide to fully wet the alumina surface, which requires around 35 to $45 \mathrm{~mJ} \cdot \mathrm{m}^{-2} 23$. Although successful bonding to oily or otherwise contaminated surfaces has been reported, where the adhesive or primer has displaced or absorbed the contaminating layer, this situation is not always possible ${ }^{24-27}$. Problems can occur when the absorbed organic contaminant modifies the boundary polymer leading to a loss in its mechanical properties ${ }^{28}$. If not displaced or absorbed, the contaminant can reduce surface wettability leading to potentially fewer bonding sites, interfacial voids or a lack of interatomic or intermolecular bonds across the interface. Inorganic contaminants such as fluorine have also been demonstrated to drastically reduce adhesion levels ${ }^{29}$.

The importance of surface roughness on both the macro- and micro- scale has also received considerable attention ${ }^{30,31}$. The role of such has not been fully explained although photoelastic studies have shown the potential for stress raisers within the bondline or interphase to redistribute loads within the joint ${ }^{32}$. To date, existing finite element analysis (FEA) models have not included the effects of micro-roughness within the bondline so its influence is currently impossible to quantify. The other advantage of surface roughness is that it makes available a much increased specific surface area over which interactions can occur. This factor could explain, to some extent, the increase in bond performance with rough surfaces.

The surface mechanical stability is highly important as the presence of an internal weak boundary layer could mean premature failure within a friable oxide or hydroxide or between the oxide/hydroxide and the metal. The presence of the weak magnesium-rich oxide on $5 \mathrm{xxx}$ series aluminium alloys is an example $\mathrm{e}^{30,33}$. Alternatively, failure within mechanically weak, thick conversion coatings on aluminium has also been reported ${ }^{34}$. It is also likely that the surface condition could influence the state of the boundary polymer in the interphasial region ${ }^{35-39}$. A change in stoichiometry, for example of the curing agent:base resin ratio in an epoxide will 
influence the mechanical properties of the adhesive and consequently its load bearing capacity $^{35,38}$.

The hydrolytic stability, or corrosion resistance of the surface is key in providing acceptable durability. In long-term exposure tests, hydration or corrosion is frequently cited as the predominant failure mechanism for improperly prepared joints exposed to hot-wet conditions ${ }^{1,2,40-42}$. In addition, a consideration of the work of adhesion theory goes some way to explaining the disruption of interfacial bonds when exposed to water.

There exists four broad categories of surface pretreatment for aluminium alloys; mechanical, chemical, electrochemical and assorted others. These pretreatments provide some or all of the above-mentioned desirable physicochemical properties to one extent or another. These are summarised briefly in the following section.

\subsubsection{Mechanical treatments}

Mechanical treatments include abrasion methods usually combined with degreasing. Pretreatment by blasting using alumina or silica grit or glass beads change the topography and chemical state of an aluminium adherend by the introduction of a "peak-and-valley" type morphology $y^{30,31,43,44}$. If fully wetted, this provides an increased area for chemical interactions. Anomalous epoxy network structures have, however, been reported to develop within such pretreated joints ${ }^{38}$. It is also recognised that excessive surface damage may be introduced by grit-blasting particularly in the softer cladding alloys.

The degreasing stage usually makes use of chlorinated solvents such as trichloroethylene, 1,1,1-trichloroethane, perchloroethylene, or dichloromethane, or alternatively, non-chlorinated solvents including methyl ethyl ketone, methanol, isobutanol, toluene or acetone. Note that, although very effective, the usage of some solvents may be restricted by legislation such as the Montreal Protocol.

Aqueous cleaners that are used for degreasing use mild acids and alkaline solutions rather than organic solvents. 
Semi-aqueous cleaners are used, incorporating less-toxic solvents, non-halogenated solvents, petroleum-based solvents or terpene solvents. These can be dissolved in water or applied in a concentrated form.

Alternatives to wet processes include atmospheric pressure plasma and laser cleaning $^{45,46}$.

In general terms, degreasing is the minimum pretreatment that is usually carried out prior to bonding. Grit-blasting or other mechanical abrasion methods are recognised as providing a useful increase in initial adhesion levels.

\subsubsection{Chemical treatments}

Surface chemical modifiers for aluminium alloys include; coupling agents, etchants and conversion coatings. The use of coupling agents, including silanes, is not covered in the present study which is primarily concerned with modification of the aluminium surface by the introduction of inorganic networks. It is sufficient to mention that a large number of studies have demonstrated the usefulness of silanes such as $\gamma$ glycidoxypropyltrimethoxysilane ( $\gamma$ GPS) when used as a primer to improve the durability of structural aluminium bonds. This has been attributed to the formation of stable, covalent bonds between the metal(oxide) and the silane and the possibility for interphase formation giving a region of intermediate modulus between the metal and polymer which facilitates stress transfer ${ }^{42,47,48}$. Silanes may be used as stand-alone processes or part of a more complex pretreatment ${ }^{49,50}$. Other coupling agents which show promising results in adhesion studies include phosphonates ${ }^{50}$ and plasma polymersised films ${ }^{51}$.

Most chemical treatments are combined with precursor degrease and desmut steps $^{52,53}$. Desmutting is used to remove gross organic and inorganic contamination usually using alkaline solutions. Such material might include: protecting or press oils; machining lubricants, and; corrosion products. Most alkaline cleaning solutions are of a propriety nature. For aluminium, they mainly consist of mixtures of sodium hydroxide, sodium carbonate, trisodium phosphate, sodium pyrophosphate or sodium metasilicate with a $\mathrm{pH}$ range between 9 and 11 . 
The purpose of an acid etch, or deoxidiser, is the further removal of all unwanted friable oxides e.g. mill scales, weak native oxides and corrosion products and to keep dissolved metals such as copper, zinc and aluminium in solution. The result of etching is to leave a thin, uniform oxide on the surface with some micro-roughness. The most effective etches incorporate mixed chromic and hydrofluoric acids. However, nonchromated acid etchants have been demonstrated to provide good adhesion results ${ }^{21,54}$.

The deoxidiser most commonly used in aerospace applications is the chromicsulphuric acid etch, also referred to as the Forest Product Laboratory (FPL) etch. A number of studies have led to an artificially-aged, optimised etch solution chemistry which incorporates dissolved aluminium and copper ${ }^{1,2,21,55}$.

Many workers have investigated alternatives to the currently-used chromatecontaining deoxidising solutions from $\mathrm{NaOH}$ and rotating brush grinding ${ }^{56}$ to the use of rare earth electrolytes ${ }^{57}$. Scalloping effects have previously been reported in rare earth etch solutions with an increase of intermetallics exposed to the surface; these could be removed by the addition of an oxidant such as $\mathrm{K}_{2} \mathrm{~S}_{2} \mathrm{O}_{8}$ or $\mathrm{H}_{2} \mathrm{O}_{2}$.

Many of the these etchants are used as stand-alone pretreatments for aluminium alloys. In terms of their effectiveness, the best performing etchants have been reported to provide a "microcomposite interphase" responsible for good initial adhesion and with durability performance in harsh environments intermediate between the mechanical and electrochemical processes ${ }^{21,58}$.

Boeing has also developed a non-chromated electrolytic deoxidiser using a low voltage DC current applied to a phosphoric acid electrolyte ${ }^{59}$. The electrolytic phosphoric acid deoxidiser (EPAD) has been show to be equivalent if not superior to chromated deoxidisers for the removal of contaminants such as outdoor storage contamination, corrosion products resulting from salt spray exposure, epoxy resin, and drill lubricants. It has been found that the preferential attack along alloy grain boundaries by the EPAD was slightly less than that found when alloys were treated with chromated deoxidisers ${ }^{59}$. This is due to the grain boundaries being more anodic 
in comparison to the rest of the matrix and so are susceptible to preferential dissolution. The operating parameters established by Boeing for the EPAD are:

- $\mathrm{H}_{3} \mathrm{PO}_{4}$ concentration...............20 $\pm 2 \%$ by weight

- Bath Temperature....................30 $\pm 3^{0} \mathrm{C}$

- Rectifier voltage.................... $7 \pm 1$ volt

- Immersion time.....................10 \pm 2 minutes

With the appropriate operating parameters, a condition exists where the oxide formation is equal to its dissolution rate. The formation/dissolution reaction continues with the EPAD electrolyte aggressively dissolving the aluminium oxide without dissolving the base aluminium.

Following deoxidising a subsequently applied conversion coating deposits a protective and/or decorative coating on metal surfaces; examples of these include phosphating, chromating and the more recently-developed titanate/zirconate hexafluoride, cerate and other processes ${ }^{60-68}$. These processes produce highly complex mixed hydrated oxides which may extend from a few nanometres to a micrometre or so in thickness. Conversion coatings can produce high energy surfaces which are highly nodular in texture and with reasonable surface passivation properties by means of both electrochemical and barrier protection methods. In terms of chromate-free conversion coatings, molybdenum has been a suggested alternative to chromium as it shares the same group in the periodic table and various anions of the form $\mathrm{MoO}_{4}{ }^{\mathrm{n}-}$ are known to inhibit the corrosion of $\mathrm{Al}$, with molybdate $\left(\mathrm{MoO}_{4}{ }^{2-}\right)$ incorporated into passive films displaying corrosion resistance to pitting ${ }^{61}$. Phosphate coatings (crystallized as hopeite, $\mathrm{Zn}_{3}\left[\mathrm{PO}_{4}\right]_{2} .4 \mathrm{H}_{2} \mathrm{O}$ ) have also been studied as alternatives to chromate pretreatments with the advantage of providing good adhesion properties. Possessing these properties, conversion coated aluminium adherends usually provide initial adhesion and durability levels comparable with acid etching and in some cases equivalent to the electrochemical pretreatments.

\subsubsection{Electrochemical treatments}


In almost all studies, however, the direct current (DC) electrochemical pretreatments give the best levels of initial adhesion and durability ${ }^{1,2,21,44,69-75}$. These processes, if optimised, provide all of the ideal physiochemical properties previously described. The three most widely used electrochemical processes used in prebond applications are phosphoric acid (PAA), chromic acid (CAA) and sulphuric acid (SAA) anodising. Other electrolytes have been studied but to a much lesser extent. CAA and PAA are both extensively used in aerospace applications ${ }^{1,2}$. Whilst numerous studies show both to perform exceptionally well it is generally regarded that CAA bonded joints perform better than PAA when exposed to corrosive environments; although the PAA process is generally preferred by the American aerospace sector. For absolute performance reasons, the CAA process has been selected as the datum in the present study.

All DC anodising procedures are complex multi-stage operations incorporating degreasing, desmutting and deoxidising stages, as described in the preceding sections, plus appropriate rinses ${ }^{52,53}$. It is also important to acknowledge the high temperature alternating current $(\mathrm{AC})$ processes which are currently the focus of much interest for high speed coil rather than batch processing ${ }^{69,76,77}$. However, at present, there are few reported studies from which to evince their performance. Thin film AC anodising in hot sulphuric acid has, however, been shown to have a number of advantages, which include; short anodising times, in the order of 5 to 20 seconds and the elimination of the degreasing or etching stages as sufficient cleaning action is caused due to the hydrogen evolved from the surface during the cathodic action of the AC current. The process is usually operated at high temperature, $\sim 80^{\circ} \mathrm{C}$ in a $15 \%$ sulphuric acid bath at current densities in the order of $10 \mathrm{~A} / \mathrm{dm}^{2}(\mathrm{rms})^{76}$. It produces a surface lacking in the characteristic scalloped features found in other anodising processes. Linear polarisation experiments used to study the corrosion properties have shown thin film AC sulphuric acid anodising provides comparable corrosion resistance to that of acid/alkaline etching and other chromate-free pretreatments such as phosphatepermanganate $^{76}$. Adhesion tests using single lap shear joints have shown thin film AC sulphuric acid anodising to give equivalent performance to the FPL etch ${ }^{77}$. However, in the present study we will consider in detail only the unsealed DC anodic oxides. 
One obvious characteristic of the anodic oxide is its porosity. The porosity of the oxide is controllable but will vary from alloy to alloy for any given anodising condition. To fully understand the formation mechanism of a porous anodic oxide, the structure of the film itself needs to be understood. The films consist of a thin, barrier layer, which is continuous over the alloy surface, above this is the porous region. The thickness of the barrier layer being cited as $\sim 1.3 \mathrm{~nm} / \mathrm{V}$ but this value can vary depending on the relative film resistivity ${ }^{78}$. The porous region which, for films grown on high purity aluminium, develops into a close-packed-hexagonal arrangement of cells comprises amorphous alumina or hydrated alumina with a central open core ${ }^{79}$. Of significance to the present study is the overall film growth mechanism. A schematic of the idealised structure grown by the 40/50V Bengough-Stuart CAA process is given in Figure 1.

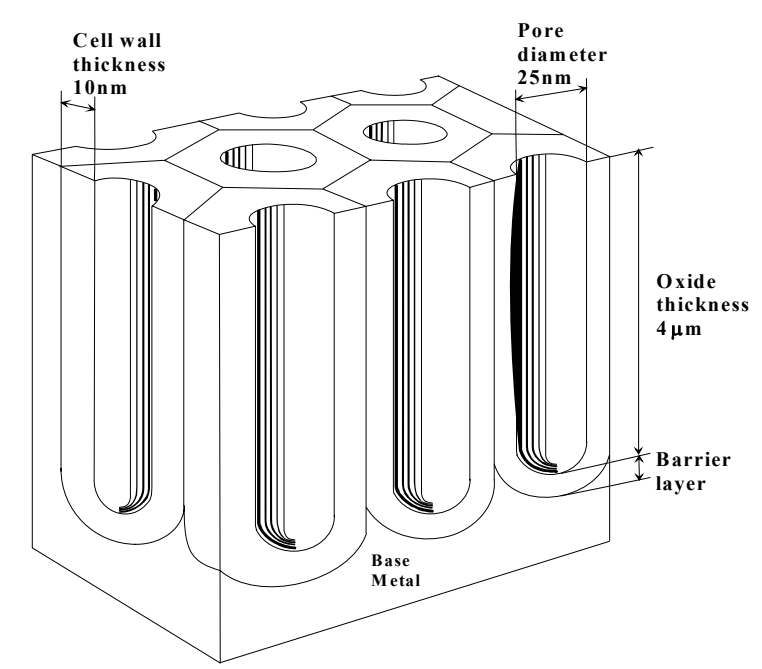

Figure 1 - To show the idealised anodic oxide structure formed on clad alloys following the 40/50V CAA process

Chemical growth of the film is reported to be possible by the solid-state migration of $\mathrm{Al}^{3+}$ and $\mathrm{O}^{2-}$ ions, which move outward and inward through the film respectively, the transport being driven by the electric field provided by the external power supply. High field ionic migration occurs with field strengths in the order of $10^{8}$ to $10^{9} \mathrm{~V} . \mathrm{m}^{-1}$ 78,79. The development of new barrier type material is due to oxidation of aluminium atoms at the metal/film interface, to form $\mathrm{Al}^{3+}$ ions, and additions of $\mathrm{O}^{2-}$ ions, derived from the electrolyte, at the film/electrolyte interface. As a result of this counter migration the new material is formed both at the metal/film and film/electrolyte 
interfaces, with about 40 per cent and 60 per cent of the film formed at the respective interfaces for film growth on high purity aluminium at high efficiency. The above studies considered electrolytes that were relatively unreactive towards the anodic film, so no aluminium species would be lost to the electrolyte. Also the use of marker ions, usually xenon, implanted into the anodic oxides has been used to further these studies to validate the above film growth mechanism. The origins of the hexagonal close packed cellular structure have also been elaborated upon in the literature ${ }^{79-83}$.

Physical properties, such as convection heat transfer, have been demonstrated to have a significant effect on anodic oxide formation. As an increase in local temperature enhances local field assisted oxide dissolution at the pore bases, and as a result acts to increase the local current density ${ }^{84}$. The applied voltages are reported to affect the barrier layer thickness, cell wall thickness, pore diameter, and the total thickness of the duplex oxide ${ }^{2}$. Solution temperature also will affect barrier layer thickness, porous layer thickness, pore diameter, and cell wall thickness, because of its effect on the solution dissolving power. In short, high temperatures produce thinner barrier and porous layers, larger pore diameters, and thinner cell walls. Low temperatures have the opposite effect ${ }^{53}$.

The effect of alloying components within the aluminium during anodising has been extensively studied ${ }^{85-89}$. In the $2 \mathrm{xxx}$ series alloys of interest in the present study, the copper-rich second phases affect adversely the film morphology and integrity, especially due to the formation of relatively large voids in the film ${ }^{79,88}$. Oxidation of copper and incorporation of $\mathrm{Cu}^{2+}$ into the anodised alumina are also associated with $\mathrm{O}_{2}$ gas evolution causing local film cracking and can lead to the nonuniform flow of ionic current through the film, due to the significant volume of $\mathrm{O}_{2}$ bubbles within the anodic film ${ }^{88,91}$. Voids can also be attributed to the high magnesium content of the $\mathrm{S}$ phase particles in 2024 alloy as during film formation the magnesium species migrate up to three times faster than $\mathrm{Al}^{3+}$ ions and are lost to the electrolyte on reaching the surface of the anodic film and in so forming voids at the alloy/film interface ${ }^{92}$.

After the film has formed at a set voltage, further barrier film thickening is not possible because the field strength for ionic transport falls to levels too low to support the co-operative mechanism of ionic movement. Thus, the current then declines to the 
so-called leakage value, associated usually with electronic current flow at the numerous flaws associated with anodic films on aluminium. At this stage of anodising there is no ionic current flow across the barrier film and hence no further formation at the film/electrolyte interface.

The advantage CAA has over PAA is the thicker amorphous oxide it produces and as a result better barrier corrosion resistance. However, Davis et $a l^{40}$ have used a range of analytical techniques to study the decomposition mechanism of PAA surfaces in an attempt to understand failure mechanisms of bonded joints. After exposure to $100 \%$ $\mathrm{RH}$ at $60^{\circ} \mathrm{C}$, complete coverage of hydration product was observed. From this the following decomposition mechanism was proposed:

$$
\mathrm{AlPO}_{4} \rightarrow \mathrm{Al}_{2} \mathrm{O}_{3} \rightarrow \mathrm{AlOOH} \rightarrow \mathrm{Al}(\mathrm{OH})_{3}
$$

The phosphate component in the PAA oxide was considered to inhibit the initial stage of the process. The CAA oxide can then be thought of as providing the better barrier corrosion protection whilst the PAA gives the best initial electrochemical corrosion resistance. Physical comparisons between CAA and PAA oxides show the PAA oxide to have a much more open porous structure which would be more easily penetrated by the adhesive in the absence of a primer, with subsequent benefits. A primer was used throughout the present study so penetration of the CAA oxide would be more likely given an optimised structure.

The boric sulphuric acid anodising (BSAA) process has advantages associated with both the CAA and PAA and as such was thought to be of interest to the present study. A BSAA process has been patented by Boeing as a direct replacement to CAA to meet environmental regulations ${ }^{93,94}$. Based upon previous work, Boeing carried out an initial screening program, with dilute sulphuric acid being the basis of four mixed acid electrolytes. The four additions to the electrolyte were: manganese sulphate, nickel sulphate-sodium phosphate, phosphoric acid, and boric acid. Boeing knew that SAA produces coatings far thicker than that of CAA, and also that high coating weights tend to reduce fatigue performance. With this in mind anodising parameters for these alternative processes, i.e. electrolyte concentrations, potential, temperature and 
anodising times were optimised to produce equivalent coating weights to that of the CAA process ${ }^{94-96}$. In these studies the phosphoric/sulphuric acid anodising process failed in corrosion testing. The remaining processes all passed the MIL-A-8625, 336hour salt spray test. The nickel sulphate-sodium phosphate/sulphuric acid anodising failed paint adhesion testing when exposed to deionised water and also fatigue testing and so was dropped. Preliminary work on the remaining two processes with respect to structural adhesive bonding was carried out but performances were inferior to that of CAA, with a tendency towards inter-oxide layer failure for the non-chromated processes, thought to be associated with the still relatively thick oxides produced. Although Boeing had identified two processes, manganese sulphate/sulphuric acid and boric acid/sulphuric acid, which performed satisfactorily in some tests BSAA was chosen for further evaluation since it does not contain any heavy metals and was expected to be a more economical bath to operate. Subsequent fatigue testing, carried out by Cree and Weidmann ${ }^{97}$, indicated that BSAA gave better bare metal fatigue performance than the $\mathrm{CAA}$, a critical result given the end-use of the required bonded structures. It should, however, be noted that in these studies the key features investigated were: corrosion resistance; paint adhesion; abrasion resistance, and; bare metal fatigue life. Boeing developed the BAC5632 BSAA process with the intension of producing an alternative pretreatment for paint adhesion and corrosion resistance and not for structural adhesive bonding. Following are the key findings from the work by Boeing:

- The solution is inexpensive to treat and dispose of and meets current EPA requirements.

- The anodic film meets all MIL-A-8625, Type I, Class I and Boeing specification BAC 5019 requirements.

- BSAA corrosion resistance is equivalent to, if not better than, chromic acid anodizing CAA in both the painted and unpainted conditions with equivalent coating weights.

- BSAA is economical and practical for manufacturing.

- BSAA paint adhesion performance is equivalent to or better than CAA.

- BSAA is able to produce thick oxide films such as MIL-A-8625, Type II, Class I by extending anodize time/potential. 
- The anodizing throwing power of BSAA is equal to that of CAA when anodizing 6061-T6 bare alloy.

In terms of adhesive bonding and corrosion resistance tests incorporating the standard BSAA process, Rohr conducted an extensive quantification test program ${ }^{98-100}$ which included, lap shear, flatwise tension, wedge crack, floating roller peel, double cantilever beam, salt spray, and wet paint adhesion tests. They concluded that the BSAA process was shown to be equivalent, and in some cases superior to, CAA as a corrosion resistant surface treatment for the adhesive bonding of aluminium. Shear, tension and wedge crack durability testing showed results statistically similar to those for CAA. However, BSAA demonstrated surprisingly superior performance over CAA in wet floating roller bell peel tests.

SIFCO have also studied the standard BSAA process by experimenting with many differing voltage/time and current density/time combinations using 2024, 6061 and 7075 aerospace alloys. They have investigated BSAA procedures using solution and gel forms, with the intention of producing a method for local repairs to damaged anodised surfaces ${ }^{101}$.

Also of relevance to the present study, work conducted using SAA electrolytes have found that a post anodising treatment has been found to enhance bond strength and durability making this process comparable to that of CAA and PAA ${ }^{73,102}$. Specifically, a phosphoric acid dip (PAD) has been used to increase the strength and durability of aluminium bonded joints. This was attributed to the hydration inhibiting properties of phosphate compounds adsorbed into the anodic oxide and also the increased mechanical interlocking due to increased surface roughness ${ }^{73}$. The PAD has also been shown to give varying results when used in combination with $\mathrm{PAA}^{73}$. Processing time has a major impact on performance, a PAD of 60 seconds shows disastrous results, but a dip time of 10 seconds shows improved wedge test results compared to standard PAA. The use of a PAD in combination with the standard BSAA process has also been considered in the present study.

\section{Other processes}


Plasma spray coatings used as pretreatments prior to adhesive bonding have shown to be equivalent if not superior on aluminium to $\mathrm{PAA}^{103,104.105}$. Other processes studied to a lesser degree and which show some useful properties, but are yet to be fully validated, include thermo-mechanical options such as: laser cleaning or desorption $^{46,106}$, deposition ${ }^{107}$ or texturing $^{108}$,and; cryoblasting ${ }^{109,110}$.

\subsection{Summary}

The 40/50V Bengough-Stuart CAA process is an aerospace industry standard pretreatment to enable structural and secondary bonding of aluminium $2 \mathrm{xxx}$ and $7 \mathrm{xxx}$ series alloys in either their clad or bare conditions. This process utilises $40 \mathrm{~g} . \mathrm{l}^{-1}$ of hexavalent chromium which is hazardous to handle and operate, and has large disposal costs. The aim of the present study, which is aimed at helping to resolve this industrial requirement and is necessarily not intended at be a purely of academic interest, is to investigate strategies for the optimisation of alternative processes which provide similar surface chemistry and topography to the CAA but utilising more benign electrolytes.

The BSAA process is presently used and accepted in the aerospace industry as a pretreatment to facilitate paint adhesion and corrosion protection of aluminium alloy components but is not recommended for structural adhesive bonding with its existing operating parameters.

The present study will use this electrolyte as a basis for a pretreatment optimised for structural bonding with a number of critical processing parameters varied compared with the standard BSAA. The BSAA anodising parameters varied include: the deoxidiser type; electrolyte concentrations; electrolyte temperature, and; the use of a post anodising dip. In addition to their influence on adhesion performance, in the present study, a range of corrosion tests have been carried out to determine the hydration resistance of the aluminium alloy surface as a function of both BSAA-based and CAA treatments. As previously discussed, one requirement of a successful pretreatment is its ability to passivate the metal. Standard practises used for the evaluation of corrosion performance include neutral salt-spray testing and filiform corrosion. In addition, a number of electrochemical techniques can be used to 
determine the degree or rate of corrosion of anodised aluminium ${ }^{77,111-114}$, results from linear polarisation studies are presented, herein.

\section{EXPERIMENTAL}

\subsection{Materials}

\subsubsection{Adherends}

The aluminium alloy chosen for the majority of studies was 2024-T3 in both bare and clad conditions.

\subsubsection{Adhesive/Primer}

The adhesive/primer combination studied was the FM 73/BR 127 system from Cytec Fiberite Ltd. FM 73 film adhesive is a single-part, toughened epoxide film adhesive supported by a polyester knit fabric scrim which controls the flow and glue line thickness during curing. BR 127 primer is a modified epoxy-phenolic consisting of $10 \%$ solids including $2 \%$ strontium chromate as a corrosion inhibiting additive. The manufacturers recommended cure cycles were used. Application of the primer was carried out within 2 hours of being dried after anodising.

\subsection{Metal processing}

\subsubsection{Standard Boric Sulphuric Acid Anodising (BSAA) Parameters}

Standard BSAA processing was carried out to Boeing specification ${ }^{94}$; following are brief details:

Initially, vapour degreasing was carried out using trichloroethylene. Desmutting was by submersion in Isoprep 44, a proprietary material from Lea Manufacturing Ltd. It is a non-caustic cleaner with a $\mathrm{pH}$ of 9.3 to 10.4 at $60^{\circ} \mathrm{C}$, with gentle agitation.

Immediately afterwards, samples were rinsed in deionised (DI) water for 3 minutes. Deoxidising was carried out by immersion in a tri-acid solution with the following composition: 
Sodium dichromate: $54 \mathrm{~g} . \mathrm{l}^{-1}$

Sulphuric acid : $\quad 100 \mathrm{ml} . \mathrm{l}^{-1}$

Hydrofluoric acid: $\quad 10 \mathrm{ml}^{-1}{ }^{-1}$

Immersion was at ambient temperature for 3 minutes. This was followed by a further rinse in DI water for 3 minutes.

Anodising was carried out using the following parameters:

Sulphuric acid: $\quad 30.5-52.0$ g. $1^{-1}$

Boric acid: $\quad 5.2-10.7$ g..$^{-1}$

Aluminium: $\quad 2.6 \mathrm{~g} \cdot \mathrm{l}^{-1}$ maximum as $\mathrm{Al}$

Copper: $\quad 155$ ppm maximum as $\mathrm{Cu}$

Chlorides: $\quad 0.1 \mathrm{~g} .1^{-1}$ maximum as $\mathrm{NaCl}$

Total chromium: $\quad$ 500ppm maximum as $\mathrm{Cr}$

Temperature: $\quad 26.7 \pm 2.2^{\circ} \mathrm{C}$

Voltage: $\quad 15 \pm 1 \mathrm{~V}(\mathrm{DC})$

Ramp rate: $\quad$ Initial voltage of 5V maximum, increased @ 5V per minute

Anodising time: $\quad 18-22$ minutes

After anodising, parts were dried thoroughly in warm air at a temperature of approximately $75^{\circ} \mathrm{C}$ prior to characterisation or priming.

\subsubsection{Modified BSAA parameters}

\subsubsection{Modified desmutting parameters}

Only one desmutting alternative was studied. This was a proprietary material from Chemetall Ltd, Pyrene 9-20 which is a non-silicated, non-etch, alkaline cleaner. The make up of the solution was $0.6 \%$ of Pyrene $9-20$ in DI water. Treatment was by full immersion for 10 minutes at $50^{\circ} \mathrm{C}$. This was followed by a 3 minute rinse in DI water prior to deoxidising

\subsubsection{Modified deoxidising parameters}


Table 1 shows the list of alternative deoxidising pretreatments studied together with a brief description of their intended use and operating parameters.

\begin{tabular}{|l|l|l|}
\hline $\begin{array}{l}\text { Alternative } \\
\text { Treatment }\end{array}$ & Description & Operating Parameters \\
\hline Pyrene 10-21 & $\begin{array}{l}\text { Highly alkaline, etch } \\
\text { cleaner }\end{array}$ & $\begin{array}{l}\text { Immersion in } 1.5 \% 10-21 \\
45^{\circ} \mathrm{C} \text { for 5 minutes }\end{array}$ \\
\hline Pyrene 14-19 & Acid cleaner, light etchant & $\begin{array}{l}\text { Immersion in } 20 \% 14-19 \\
\text { at } 60^{\circ} \mathrm{C} \text { for 3 minutes }\end{array}$ \\
\hline Pyrene 7-77 & $\begin{array}{l}\text { Chromium free treatment } \\
\text { prior to painting }\end{array}$ & $\begin{array}{l}\text { Immersion in } 2.5 \% 7-77 \text { at } \\
25^{\circ} \mathrm{C} \text { or } 50^{\circ} \mathrm{C} \text { for 5 minutes }\end{array}$ \\
\hline Pyrene 14-73 & $\begin{array}{l}\text { Similar to } 14-19 \text { with } 9 \mathrm{x} \\
\text { fluoride content }\end{array}$ & $\begin{array}{l}\text { Immersion in } 20 \% 14-73 \\
\text { at ambient temp for 10 } \\
\text { minutes }\end{array}$ \\
\hline Chemcid 2218 & $\begin{array}{l}\text { Non-chromated liquid } \\
\text { deoxidiser }\end{array}$ & $\begin{array}{l}\text { Immersion in } 20 \% 2218 \text { at } \\
30^{\circ} \mathrm{C} \text { for } 10 \text { minutes }\end{array}$ \\
\hline $\begin{array}{l}\text { Phosphoric acid } \\
\text { deoxidiser (PAD) }\end{array}$ & $\begin{array}{l}\text { Electro-chemical } \\
\text { deoxidiser }\end{array}$ & See below \\
\hline
\end{tabular}

Table 1 - A summary of alternative deoxidisers studied

Note that the Pyrene and Chemcid processes are proprietary materials from Chemetall Ltd, whilst phosphoric acid deoxidising (PAD) uses a generic solution. PAD was carried out under the following conditions:

Concentration: $\quad 20 \pm 2 \%$ by weight of phosphoric acid.

Operating temperature: $\quad 30 \pm 2^{\circ} \mathrm{C}$.

Anodic potential: $\quad 7.0 \pm 2 \mathrm{~V}$

Deoxidising time: $\quad 10 \pm 2$ minutes 
All deoxidising treatments were followed by a 3 minute rinse in DI water prior to anodising.

\subsubsection{Modified anodising parameters}

The following three variables were altered: electrolyte temperature, electrolyte acid concentrations and the anodising potentials. The electrolyte temperature was varied within the range 15 to $40^{\circ} \mathrm{C}$. Boric acid concentrations were varied between 2.5 to 10 g. $1^{-1}$ and sulphuric acid between 20 and 60 g. $1^{-1}$. The anodic potentials were investigated in the range 15 to $30 \mathrm{~V}$.

\subsubsection{Post anodising treatment}

The PAD was carried out using the following conditions:

Concentration: $\quad 20 \pm 2 \%(w t)$ phosphoric acid.

Operating temperature: $\quad 30 \pm 2{ }^{\circ} \mathrm{C}$.

Dip time: $\quad 10 \pm 2$ minutes

This was followed by a 3 minute rinse in DI water then air drying.

\subsubsection{Standard CAA Parameters}

This was carried out according to Short Bros.P.SPEC.410 a version of the BengoughStuart process ${ }^{115}$. Desmutting and degreasing were carried out as described in Section 2.2.1. Deoxidising was carried out using the 'optimised' FPL acid etch; the make up of this solution was as follows:

Sodium dichromate: $\quad 60$ g. $\mathrm{l}^{-1}$

Sulphuric acid: $\quad 304 \mathrm{~g}^{-1} \mathrm{l}^{-1}$

Aluminium 2024 alloy: $\quad 1.5 \mathrm{~g} \cdot \mathrm{l}^{-1}$ (minimum)

The operating bath temperature was $60^{\circ} \mathrm{C}$ with gentle agitation using an electromagnetic stirrer. This was followed by a rinse stage for 3 minutes in DI water.

The specified anodising parameters are listed below. 
Chromic acid: $\quad 30.5-50.0 \mathrm{~g} \cdot 1^{-1}$

Chlorides: $\quad 0.20 \mathrm{~g} . \mathrm{l}^{-1}$ maximum as $\mathrm{NaCl}$

Sulphates: $\quad 0.50 \mathrm{~g} .1^{-1}$ maximum as $\mathrm{Na}_{2} \mathrm{SO}_{4}$

Temperature: $\quad 40 \pm 2^{\circ} \mathrm{C}$

Voltage: $\quad 40-50 \pm 1 \mathrm{~V}(\mathrm{DC})$

Ramp rate: $\quad$ See figure 1

Anodising time: $\quad 35-45$ minutes

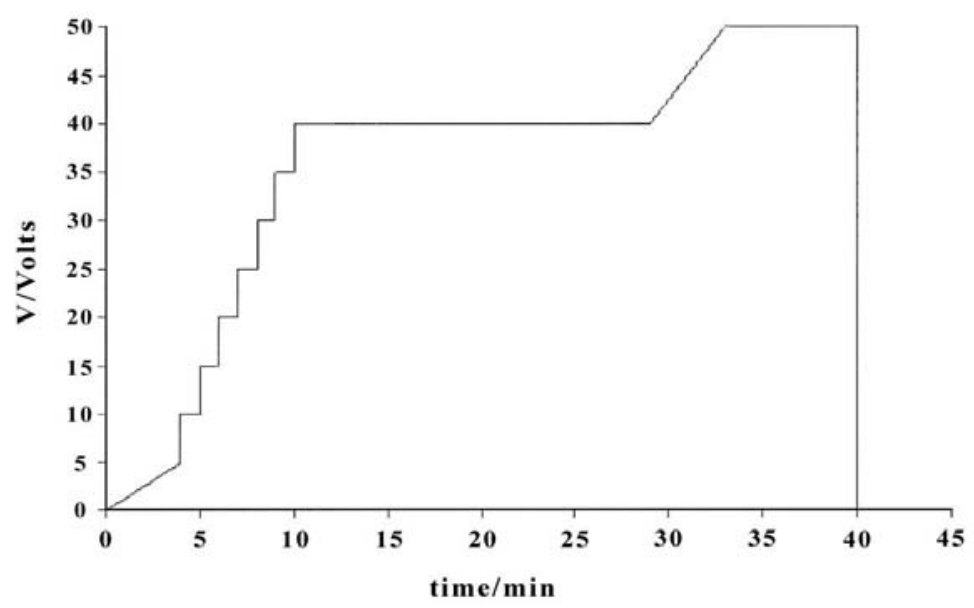

Figure 2 - Operating cycle for standard CAA 40/50V process

After anodising, parts were rinsed in DI water at ambient temperature for 5 minutes then dried thoroughly in warm air at a temperature of approximately $40^{\circ} \mathrm{C}$ for a maximum of 60 minutes.

\subsection{Surface Analysis Methods}

\subsubsection{Scanning electron microscopy (SEM)}

SEM was carried out using a Cambridge Steroscan 360 instrument operating with a primary beam energy of $20 \times 10^{3} \mathrm{~V}$ and a current of approximately $250 \times 10^{-9} \mathrm{~A}$. In all cases, samples were sputter gold coated prior to analysis. Limited EM was also carried out on a LEO 1530VP instrument. 


\subsubsection{Transmission Electron Microscopy (TEM)}

The instrument used was a Jeol 100CX operating with a primary beam energy of $100 \times 10^{3} \mathrm{eV}$. The machine was operated in scanning (STEM) mode. All samples were sputter gold coated prior to analysis.

\subsubsection{Auger Electron Spectroscopy (AES)}

A Varian AES spectrometer was used, operating with a primary beam energy of $3 \times 10^{3} \mathrm{eV}$ into an area approximately $100 \times 10^{-6} \mathrm{~m}$ across. Depth profiling was carried out using sequential argon-ion bombardment with a beam energy of $3 \times 10^{3} \mathrm{eV}$ and a current density of $75 \times 10^{-6} \mathrm{~A} . \mathrm{cm}^{-2}$. Experimentally derived sensitivity factors were used for quantification based upon $\mathrm{Al}_{2} \mathrm{O}_{3}$ and $\mathrm{MgO}$ reference materials. $\mathrm{An}$ experimentally derived etch rate was used based upon an $\mathrm{Al}_{2} \mathrm{O}_{3}$ conversion coating on an aluminium 5251 alloy substrate. The etch rate applied was 24 nm.minute ${ }^{-1}$. AES was used for surface chemical characterisation and depth profiling.

\subsubsection{X-ray Photoelectron Spectroscopy (XPS)}

XPS was carried out using a VG ESCALAB MkI operating using $\mathrm{AlK}_{\alpha}$ X-rays with an anode voltage of $10 \times 10^{3} \mathrm{~V}$ and filament current of $20 \times 10^{-3} \mathrm{~A}$. Quantification was achieved using theoretically derived relative sensitivity factors. Both survey and high resolution spectra were carried out on all the samples. XPS was used for surface chemical characterisation and the failure mode analysis of fractured joints.

A number of additional characterisation experiments were also carried out including atomic force microscopy (AFM), stylus profilometry, Fourier transform infra-red (FTIR) analysis and water contact angles. The results of these experiments are mentioned in the following section but not discussed in detail; consequently the experimental procedures used have not been elaborated upon.

\subsection{Mechanical Test Methods}

\subsubsection{Single lap shear (SLS) joint testing}


SLS joints were prepared with various surface treatments using 2024-T3 clad alloy coupons, their dimensions are shown below, Figure 3.

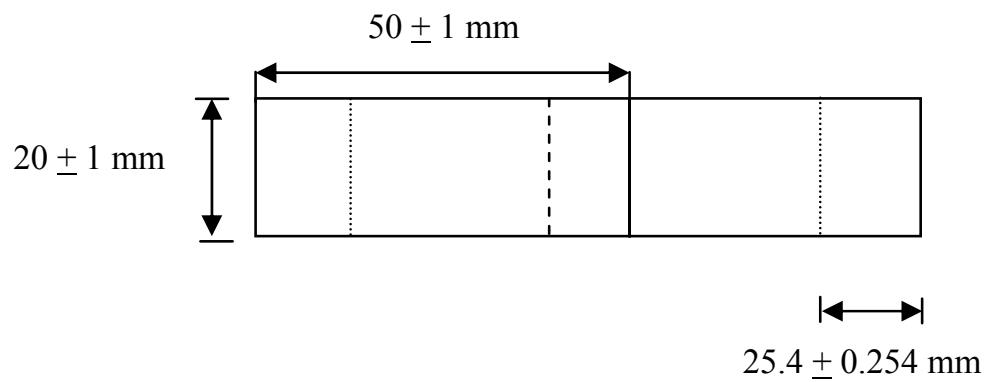

(Area in Test Grips)

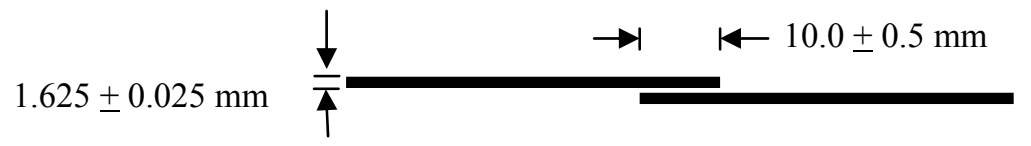

Figure3 - Dimensions of joints. (a) plan view, (b) side view

Joints were tested on a Hounsfield H20K-W instrument with a $50 \mathrm{kN}$ load cell using a crosshead speed of $6 \mathrm{~mm}$ per minute. Maximum mean loads to failure were recorded. Five replicates were used in all cases.

\subsubsection{Aged single lap shear (SLS) joint testing}

SLS joints were exposed to $60^{\circ} \mathrm{C}$ and $100 \%$ relative humidity (RH) for periods of 30 , 60 , and 120 days. To prevent secondary bonds from reforming none of the joints were allowed to dry before being tested. Three replicates were prepared for each test variable, their dimensions are shown below; see Figure 4. Testing was carried out using a Lloyd instrument with a $50 \mathrm{kN}$ load cell with a crosshead speed of $6 \mathrm{~mm}$ per minute.

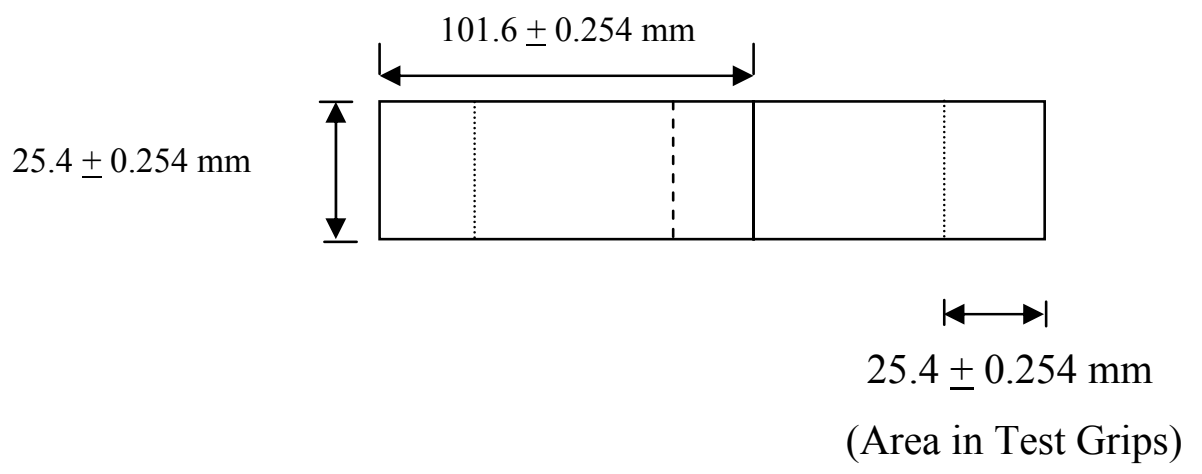




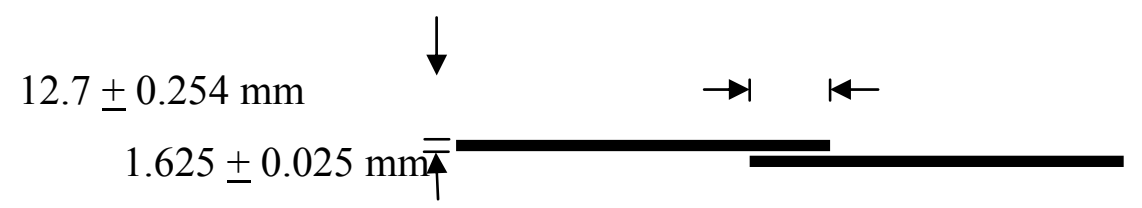

Figure 4 - Dimensions of joints. (a) plan view, (b) side view

\subsubsection{Wedge testing}

Initially, wedge test joints were prepared according to ASTM D3762-79 ${ }^{116}$, these gave highly variable results. Further to this, non-standard wedge test joints were prepared. In the non-standard wedge tests individual coupons, measuring $25.4 \times 150 \times 3 \mathrm{~mm}$, were machined before any surface pretreatment was carried out. Ten replicates were prepared for each treatment condition. The treated coupons were then primed and bonded in the usual way and mounted in specially designed jigs for curing. Once cured, excess fillets were polished off so that the bondline could be clearly defined. In addition, controlled insertion of the wedge was carried out using a Hounsfield H20K$\mathrm{W}$ tensometer at a rate of $50 \mathrm{~mm}$ per minute. After the wedges had been inserted a stabilization period of one hour was allowed for crack growth to normalise. In all cases wedge test joints were placed in a humidity chamber at $60^{\circ} \mathrm{C}$ and $100 \% \mathrm{RH}$ for periods up to 100 hours.

\subsubsection{Fatigue testing}

Cyclic load fatigue testing of bonded joints was carried out with the use of SLS joints prepared using 2024-T3 clad alloy and anodised using the various anodising processes. The joints, shown in Figure 5, were immersed in DI water at room temperature during testing, with a load range of 100 to $1000 \mathrm{~N}$ at a frequency rate of $10 \mathrm{~Hz}$ until failure of either the joint or adherend occurred.

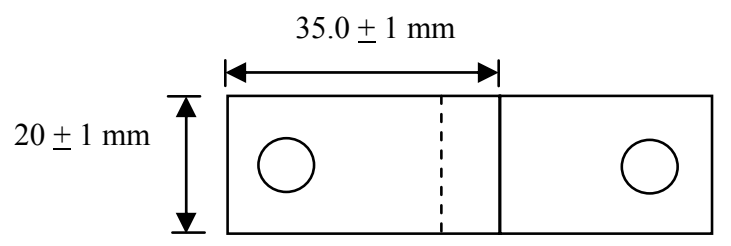




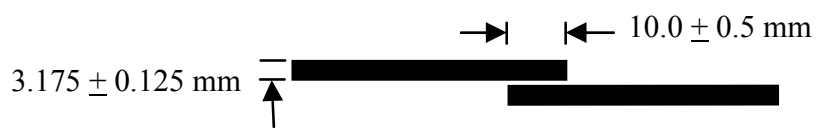

Figure 5 - Dimensions of single lap shear joints for cyclic fatigue testing

\subsubsection{Coating weight testing}

Coating weights were determined using the procedure detailed in ASTM B137-95 ${ }^{117}$ in which an anodised test panel of known surface area was weighed. The anodic oxide was then removed with a stripping solution of phosphoric-chromic acid solution containing 20g. $1^{-1}$ chromic acid anhydride, $35 \mathrm{ml} .1^{-1}$ othophosphoric acid in DI water, maintained at a temperature of about $100^{\circ} \mathrm{C}$. After rinsing and drying the test panel was re-weighed. The coating weight is then simply the surface area of the panel divided by the difference in weight before and after stripping.

\subsubsection{Neutral salt spray testing}

Test coupons were prepared and tested according to the ASTM B $117^{118}$ standard, in which 2024-T3 bare $25.5 \times 7.5 \times 0.1 \mathrm{~cm}$ panels were exposed to $3.5 \%$ sodium chloride solution in a salt spray cabinet, operated at $35^{\circ} \mathrm{C}$. Panels were mounted at an incline of $6^{\circ}$ from vertical. Specimens were examined after 336 hours exposure for evidence of corrosion and classified as a "pass" or "fail", depending on the number and size of corrosion pits. The criteria for this classification were that the panels should not show more than five isolated spots or pits, none of which should be larger than $0.8 \mathrm{~mm}$ in diameter.

\subsubsection{Filiform corrosion testing}

This was carried out according to the procedure detailed in BS EN ISO $4623^{119}$. Filiform corrosion test panels measuring $15 \times 7.5 \times 0.1 \mathrm{~cm}$ were prepared with a CMS 565-01 primer and CMS 565-02 topcoat applied. Eight panels per alloy and surface pretreatment were used for evaluation. A scribe was used to produce scratches $1 \mathrm{~mm}$ wide, in the horizontal and vertical directions on the panel. Filiform corrosion was initiated by exposure to $\mathrm{HCl}$ vapour for 1 hour and the panels were then exposed to $42^{\circ} \mathrm{C}$ and $82 \% \mathrm{RH}$ for 1000 hours. The extent of filiform corrosion was quantitatively 
assessed by measuring the lengths and areas of the filaments that developed on each scribe using an image analyser.

\subsubsection{Linear polarisation experiments}

Electrochemical measurements were carried out using a Potentiostat-galvanostat controlled by a personal computer with an Auto Tafel data logger. A Faraday cage was used to isolate the electrochemical cell from interference. All potentials were measured with reference to a Standard Calomel Electrode (SCE). The SCE was also isolated from the cell using a Luggin-Haber capillary filled with a solution of saturated potassium chloride. A solution of $3.5 \%$ sodium chloride $(\mathrm{NaCl})$ in $\mathrm{DI}$ was used which was at room temperature with no stirring or agitation. Masking of samples was carried out with chemically inert tape so that no bare edges were exposed to the solution, avoiding any edge effects. Also, in all cases masking was carried out such that a single sided surface area of $2 \mathrm{~cm}^{2}$ was exposed to the solution. The counter electrode or auxiliary electrode used was a platinum mesh to maximise surface area. The time dependence of the open circuit potential between the test sample electrode and the reference electrode was monitored for the different alloys and a series of times were determined for each alloy to achieve equilibrium, this value the corrosion potential, was recorded and compared to published values. Both anodic and cathodic polarisation scans were performed on a range of treated samples. The electrode potential was varied using a sweep rate of $0.1 \mathrm{mV} . \mathrm{sec}^{-1}$ and polarisation curves were determined from the rest potential to an anodic potential of $3000 \mathrm{mV} / \mathrm{SCE}$ or from rest potential to a cathodic potential of $-3000 \mathrm{mV} / \mathrm{SCE}$. A different test piece, of the same sample, was used for the anodic and cathodic sweeps.

\section{RESULTS}

The results obtained are divided into three sections; surface characterisation, mechanical testing and corrosion test results.

\subsection{Surface characterisation}


The results presented in this section, illustrate the physicochemical changes introduced after various stages of processing. Initially, the minimum surface treatment of degreasing was carried out on all the alloys. Further to this, materials were then characterised as a function of different deoxidising processes. The influence of varying the standard BSAA anodising processes was then studied, followed by an investigation into the effects of post anodic etching.

\subsubsection{Degreased-only aluminium alloy}

SEM studies showed the degreased-only surface of both clad and bare 2024-T3 alloy to be largely planar with only slight rolling lines and scratches visible. Figure 6 illustrates this for the 2024-T3 clad material. This was further confirmed with the use of both AFM and profilometry; see Table 2.

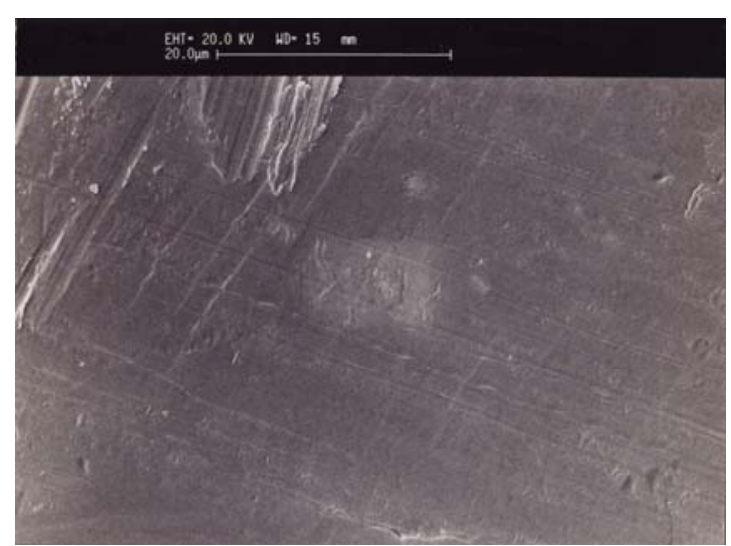

(a)

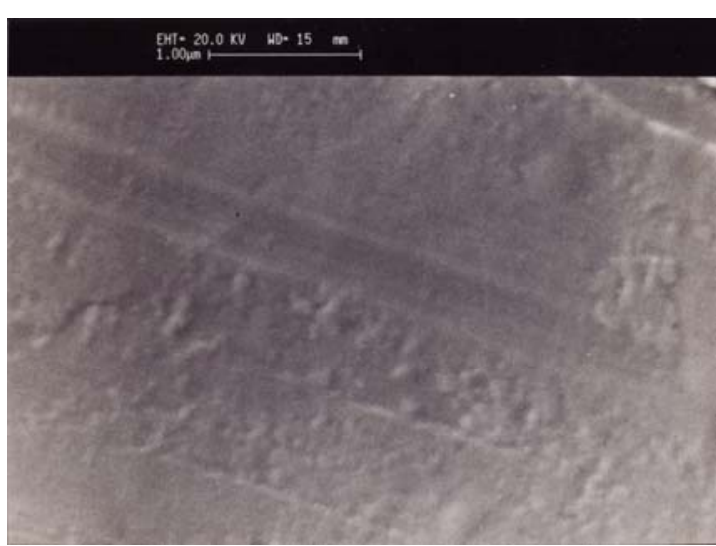

(b)

Figure 6 - Low (a) and high (b) magnification SEM images of degreased-only 2024T3 clad alloy surface

\begin{tabular}{|c|c|c|c|}
\hline Treatment & Direction & $\mathrm{R}_{\mathrm{a}}\left(\mathrm{x} 10^{-6} \mathrm{~m}\right)$ & $\mathrm{R}_{\mathrm{t}}\left(\mathrm{x} 10^{-6} \mathrm{~m}\right)$ \\
\hline \multirow[t]{2}{*}{2024 clad } & $\mathrm{T}$ & 0.061 & 0.460 \\
\hline & $\mathrm{L}$ & 0.083 & 0.602 \\
\hline \multirow[t]{2}{*}{2024 bare } & $\mathrm{T}$ & 0.065 & 0.423 \\
\hline & $\mathrm{L}$ & 0.074 & 0.584 \\
\hline
\end{tabular}

Table 2 - Profilometry data for degreased-only alloy surfaces 
Note that in Table 2, "T" and "L" refer to the direction of travel of the stylus, these being transverse to and along the direction of the visible rolling lines respectively. The $\mathrm{R}_{\mathrm{a}}$ parameter gives the mean deviation from the mean line whereas the $\mathrm{R}_{\mathrm{t}}$ parameter gives the maximum peak-to-valley distance on the surface. The values quoted in Table 4 are representative of a number of scans carried out on each sample.

AES was used to determine surface and sub-surface compositions of the degreasedonly alloys. Full depth profiling was carried out to determine the composition and thickness of the contaminating layer and native oxide on the degreased-only material. Surface compositions are given in Table 3.

\begin{tabular}{|l|l|l|l|l|l|l|l|}
\hline Sample & $\mathrm{O}$ & $\mathrm{Al}$ & $\mathrm{Ca}$ & $\mathrm{Mg}$ & $\mathrm{Cl}$ & $\mathrm{C}$ & $\mathrm{S}$ \\
\hline 2024 clad & 60.1 & 31.4 & 1.2 & 0.0 & 0.0 & 7.3 & 0.0 \\
\hline 2024 bare & 36.1 & 6.0 & 0.0 & 23.8 & 2.9 & 31.1 & 0.0 \\
\hline
\end{tabular}

Table 3 - Summary of surface compositions from AES data (atom \%) for degreasedonly substrates

AES showed the thickness of the native oxide layers for the degreased-only alloy substrates to be approximately $20 \mathrm{~nm}$ for the 2024 clad and $117 \mathrm{~nm}$ for the bare alloy. Here the thickness of the oxide is defined as the depth at which the oxygen levels decrease to half that of its maximum value in the AES depth profiles. Figure 7 
illustrates the full AES depth profile for the clad alloy.

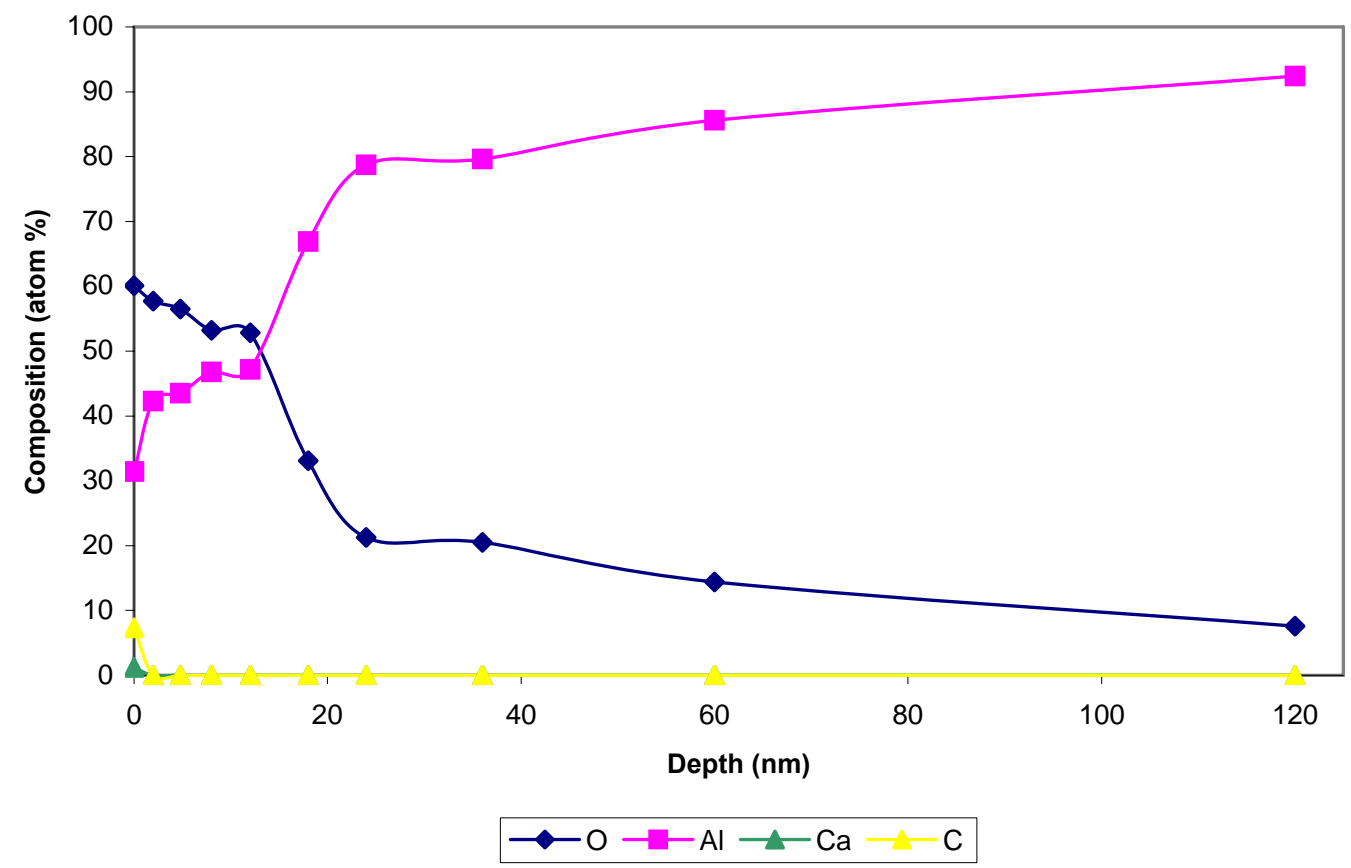

Figure 7 - AES depth profile of surface oxide on 2024-T3 clad aluminium alloy sample

\subsubsection{Standard surface pretreatments prior to anodising}

\subsubsection{Desmutting - Isoprep 44}

Isoprep 44, the alkaline cleaning procedure used for both the CAA and BSAA processes was shown to have little effect on the surface topography, as indicated by both SEM and AFM. AES data indicated no change in oxide thickness on the 2024T3 clad treated surface, however, there was evidence of residual silicon. This is not unexpected, as Isoprep 44 is a silicated process.

\subsubsection{Deoxidising - Optimised FPL and Tri-acid etching}

SEM images of the 'optimised' FPL etch, used in the CAA process, showed a characteristic scalloped texture, see Figures $8 \mathrm{a}-\mathrm{b}$, consistent with that reported in the liturature. The scallops were approximately 0.5 to 1 micrometre $(\mu \mathrm{m})$ in diameter. The rolling lines were mainly removed during etching and the scalloping probably relates to the cellular structure of the alloy, with ridges developing where sub-grains intersect the surface of the alloy. Pocius et al have observed the number of etch pits to 
be much greater in the case of the 2024-T3 bare alloy than in the clad alloy. Both Pocius and Venables have also, separately, observed segregation of copper at the metal/oxide interface of the bare 2024-T3 alloy using the FPL etch, something not observed in the present study.

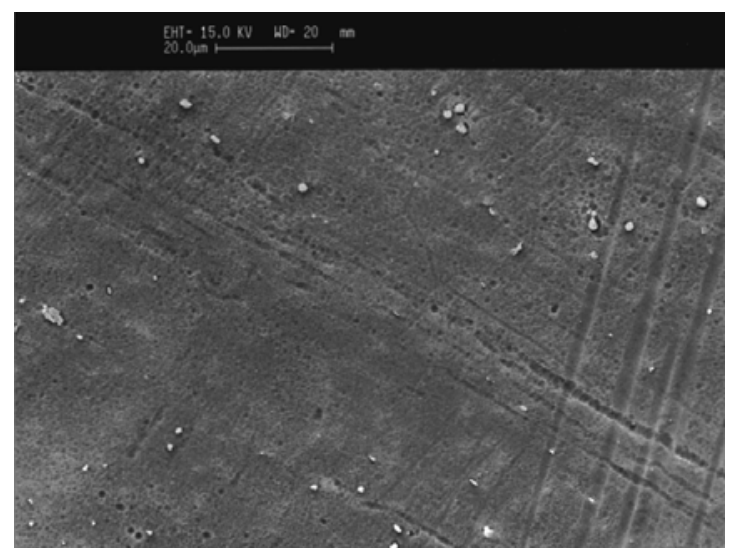

(a)

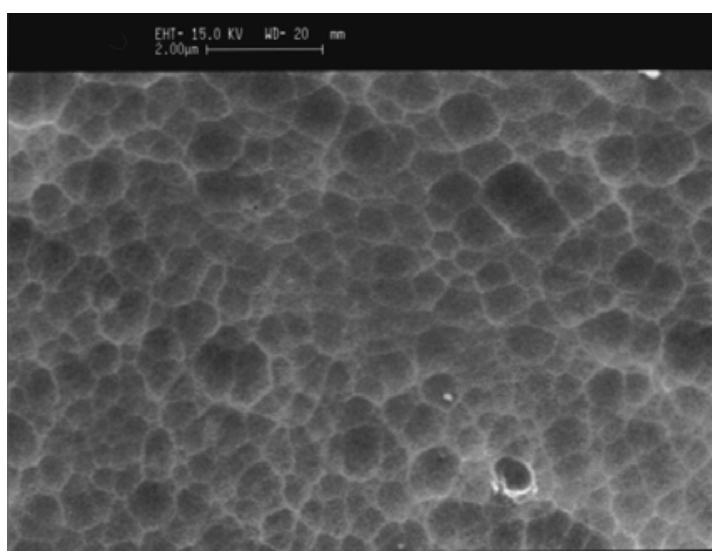

(b)

Figure 8 - Low (a) and high (b) magnification SEM images of 'optimised' FPL etched 2024-T3 clad alloy surface

AES data indicated an oxide thickness close to $20 \mathrm{~nm}$ with low levels of surface contamination, typically in the range 10 to $15 \%$.

In the case of the tri-acid etch as used in the BSAA process the same scalloped features were present; see Figure 9. These again ranged from 0.5 to $1 \mu \mathrm{m}$ in diameter, the size of which, again, seem to be independent of alloy type.

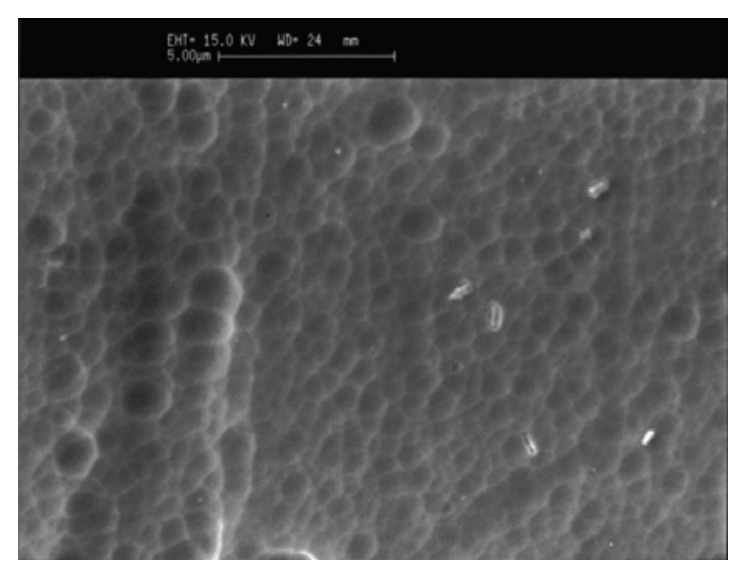


Figure 9 - High magnification SEM image of Tri-acid etched 2024-T3 clad aluminium alloy surface

AES data of the tri-acid etched surface indicated a thicker oxide, extending to approximately 40nm, with evidence of fluorine present on the surface and uppermost part of the oxide. Again, only low levels of carbon, typically in the range of 5 to $10 \%$, were observed indicating sub-monolayer coverage of contaminant. This value for the oxide thickness is consistent with that commonly observed for aluminium alloys etched in chromic acid-based media.

Water contact angle measurements indicated the cleaning efficiency of the acid etch treatments in comparison to that of the degreased-only surface; see Table 4. In this table the low contact angle of less than $10^{\circ}$ on the acid etched surfaces are indicative of a high surface energy and therefore low level of contamination. In contrast, the degreased-only surface gave a much higher contact angle indicative of a low surface energy, relatively highly contaminated material.

\begin{tabular}{|l|l|}
\hline Treatment & Contact Angle $\left(^{0}\right)$ \\
\hline Degreased & $40-70$ \\
\hline FPL etch & $<10$ \\
\hline Tri-acid etch & $<10$ \\
\hline
\end{tabular}

Table 4 - Water contact angles on various treated 2024 clad alloy surfaces

\subsubsection{Non-standard deoxidisers prior to anodising}

A number of processes were investigated, as they were considered to be capable of replacing FPL/Tri-acid etching by producing the same texture and or chemistry but without the use of hexavalent chromium. In the case of these alternative processes only the 2024-T3 clad alloy was studied.

Pyrene 10-21, Figure 10a, gave a very slightly scalloped surface but left some debris present on the surface, suggesting inadequate rinsing or cleaning power for this solution. 
Pyrene 14-19, Figure 10b, produced a scalloped surface similar to that of the FPL but left relatively high levels of debris remaining on the surface; again this suggests either a more powerful deoxidising solution is required or there is an inadequate rinse stage. AES depth profile data indicated an oxide thickness of approximately $5 \mathrm{~nm}$. This thin oxide is suspected to be due to the formation of insoluble phosphates, which are inhibiting the formation of other oxides.

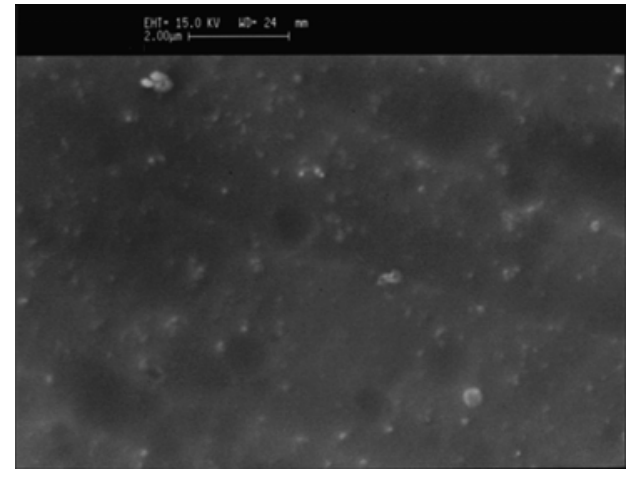

(a)

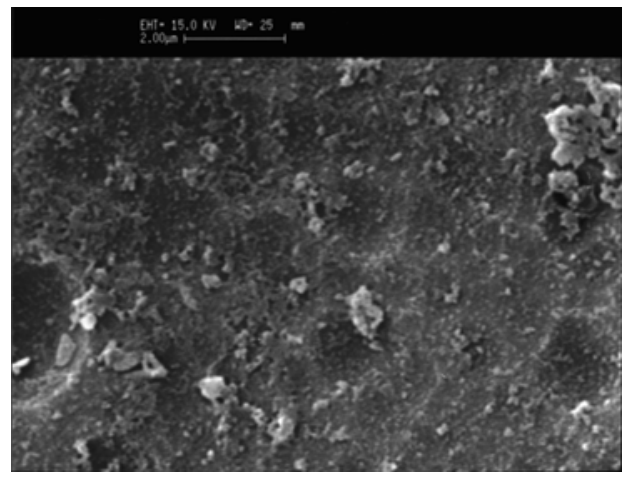

(b)

Figure 10 - SEM images of Pyrene 10-21 (a) and Pyrene 14-19 (b) deoxidised 2024T3 clad alloy surfaces

SEM plan views of the 2024-T3 clad alloy surface following the Pyrene 14-73 and Chemcid 2218 treatment are shown in Figure 11. The Pyrene 14-73 surface showed similar scalloped texture to that of the FPL etch. The Chemcid 2218 was shown to form only a partial film.
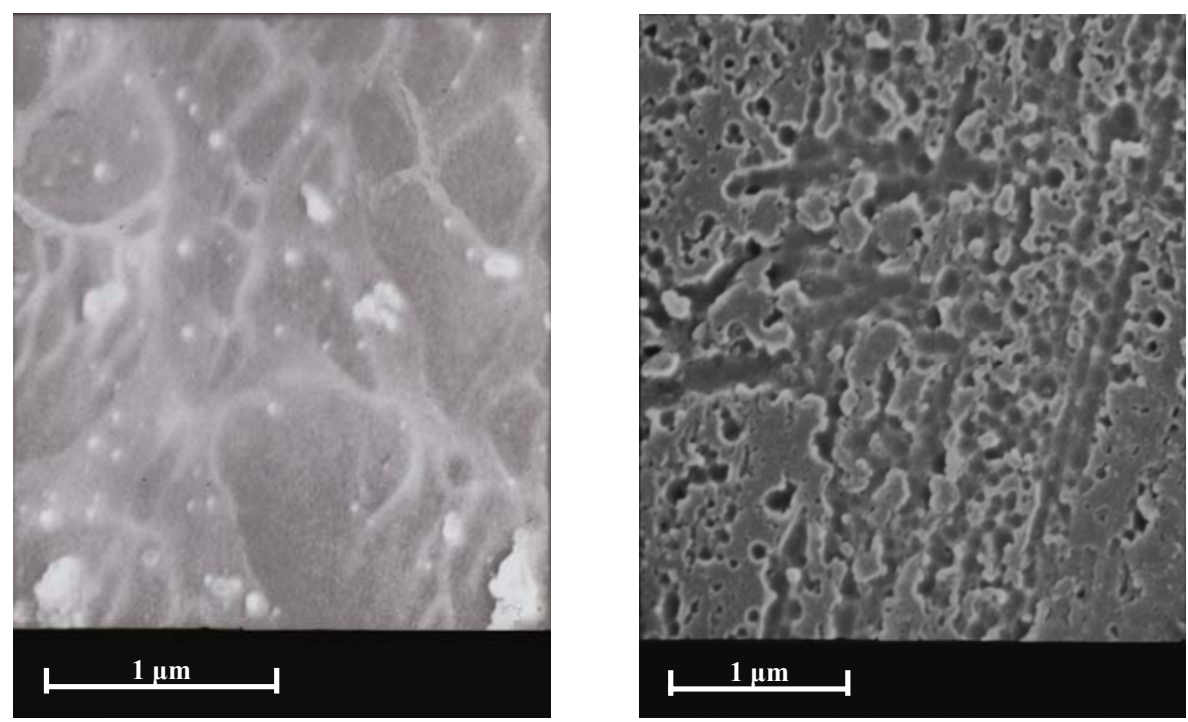
Figure 11 - Surface topography of Pyrene 14-73 (a) and Chemcid 2218 (b) deoxidised 2024-T3 clad alloy

In contrast, SEM images of the electrolytically phosphoric acid deoxidised surface, Figure 12, showed a distinctive rough nodular texture.

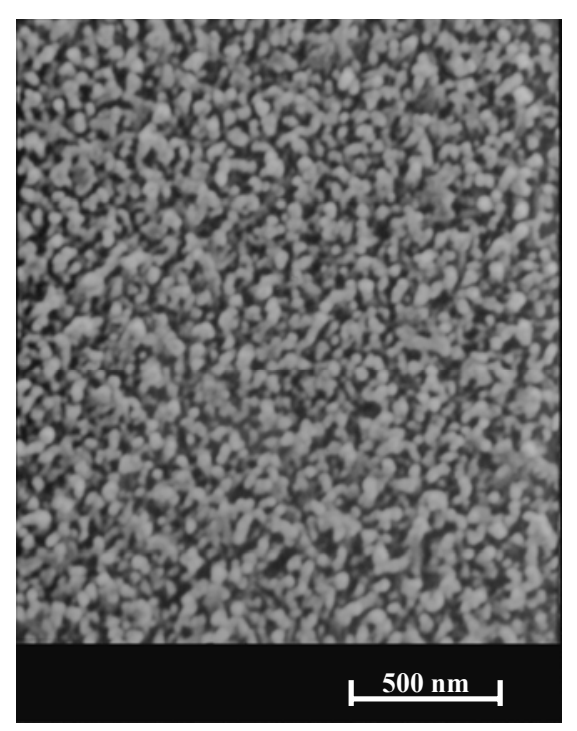

Figure 12 - Surface topography of the electrolytically phosphoric acid deoxidised 2024-T3 clad alloy

Profilometry data for the electrochemically phosphoric acid deoxidised surface is given in Table 5 .

\begin{tabular}{|l|l|l|l|}
\hline Treatment & Direction & $\mathrm{R}_{\mathrm{a}}\left(\mathrm{x} \mathrm{10^{-6 } \mathrm { m } )}\right.$ & $\mathrm{R}_{\mathrm{t}}\left(\mathrm{x} 10^{-6} \mathrm{~m}\right)$ \\
\hline EPAD & $\mathrm{T}$ & 0.097 & 0.700 \\
\hline & $\mathrm{L}$ & 0.094 & 0.666 \\
\hline
\end{tabular}

Table 5 - Profilometry data for phosphoric acid deoxidised 2024 clad alloy surfaces

\subsubsection{Standard anodising processes}

\subsubsection{CAA}


In the case of the 2024-T3 clad substrate the CAA treated surfaces appeared to be relatively uniform with a reasonably compact film formed and few voids present in the coating, Figure 13a. At higher magnification, Figure 13b, the scalloped texture is still evident from the deoxidised surface. However, the 2024-T3 bare substrate produces, to varying degrees, a fractured film surface; see Figure 14. These observations differ from the majority of reported studies, which state comparable oxide morphology for both the clad and bare alloys.

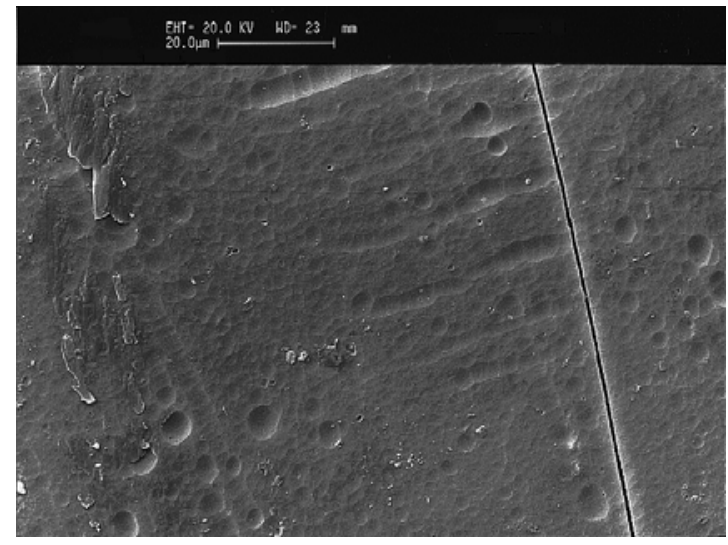

(a)

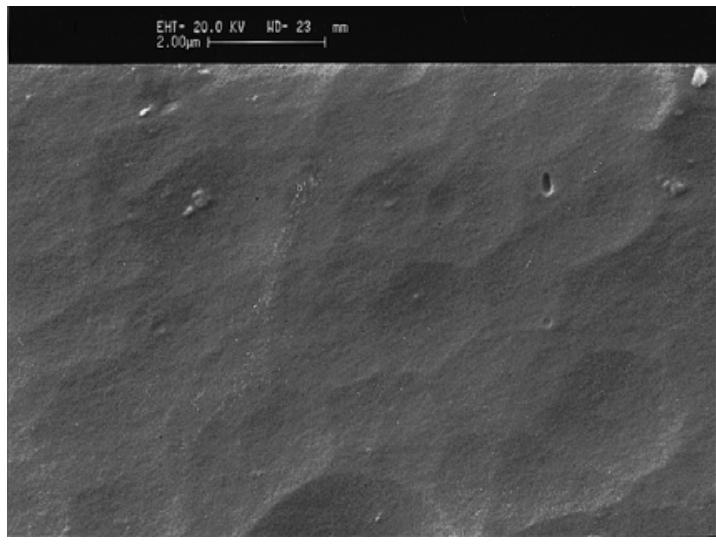

(b)

Figure 13 - Low (a) and high (b) magnification SEM images of CAA processed 2024T3 clad alloy surface

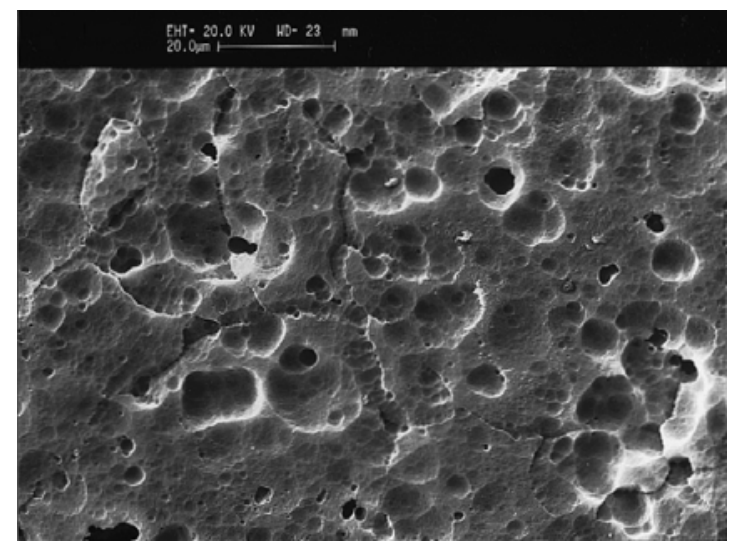

Figure 14 - Low magnification SEM image of CAA processed 2024-T3 bare aluminium alloy surface

Figures $15 \mathrm{a}-\mathrm{b}$ show the surface topography of the CAA processed alloys at higher magnifications. Only the 2024-T3 clad alloy exhibited the classical porous structure 
reported in the literature. For this alloy, pore and cell wall dimensions at the surface can be estimated as being in the range of 15 to $30 \mathrm{~nm}$ and 10 to $15 \mathrm{~nm}$ respectively. Pore densities for the 2024-T3 clad alloy varied from 5 to $10 \times 10^{10} \mathrm{~cm}^{-2}$. Close inspection of the CAA bare 2024-T3 film shows that the expected hexagonal pore array was not present, instead a more random array exists. Furthermore, it is evident that a number of pores have merged with their nearest neighbours and that there exists a very nodular, "sponge-like" structure. This latter observation has also been noted by Zhou and Thompson et al.

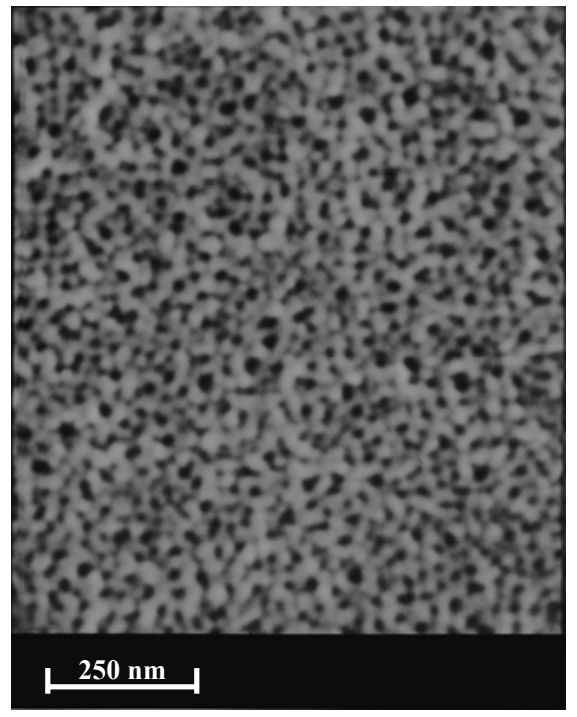

Figure15a - Surface topography of CAA processed 2024-T3 clad alloy

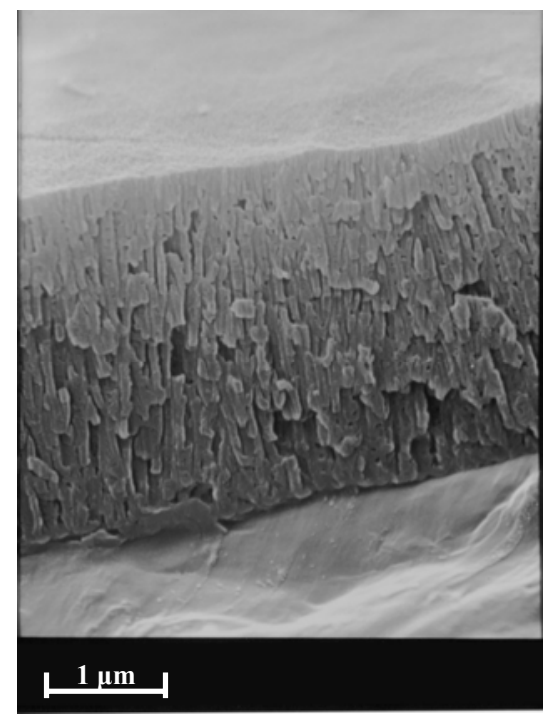

Figure 15b - Cross-sectional image of CAA processed 2024-T3 clad alloy 
Anodic oxide thickness values obtained from cross-sectional views of the CAA aluminium, are in the order of $3.0 \pm 0.5 \mu \mathrm{m}$ for the $2024-\mathrm{T} 3$ clad alloy and $2.0 \pm 0.5 \mu \mathrm{m}$ for the 2024-T3 bare alloy. Studies using AES analysis showed no organic material present on the CAA treated surfaces, Table 6. The detection limits for carbon in AES was calculated to be approximately 0.5 atomic percent; this value was based on the signal:noise ratio in the region of the AES spectra where the C Auger peak at $\sim 272 \mathrm{eV}$ would be expected to be present.

\begin{tabular}{|l|l|l|l|l|}
\hline Alloy type & \multicolumn{2}{|l|}{ CAA 40/50V } & \multicolumn{2}{l|}{ BSAA } \\
\hline & O & Al & O & Al \\
\hline 2024-T3 bare & 63.4 & 36.6 & 57.5 & 42.5 \\
\hline 2024-T3 clad & 63.6 & 36.4 & 61.1 & 38.9 \\
\hline
\end{tabular}

Table 6 - Summary of AES data showing no contamination on anodised surfaces

Water contact angle measurements further indicated a highly clean surface provided by the anodising treatments in comparison to the surface treatments prior to anodising, Table 7. Typical standard deviations were $\pm 3^{0}$. The undoubted influence of surface texture on these water contact angles was not taken into account. Most significantly, these surfaces clearly fully wetted immediately upon contact with water.

\begin{tabular}{|l|l|}
\hline Treatment & Contact Angle $\left(^{0}\right)$ \\
\hline CAA & $<5$ \\
\hline BSAA & $<5$ \\
\hline
\end{tabular}

Table 7 - Water contact angles on anodised 2024 clad alloy surfaces

\subsubsection{BSAA}

On the macro-scale the BSAA anodised 2024-T3 clad and bare alloys showed very similar topography to the CAA surface with the same substrate; see Figures 16 and 17. 


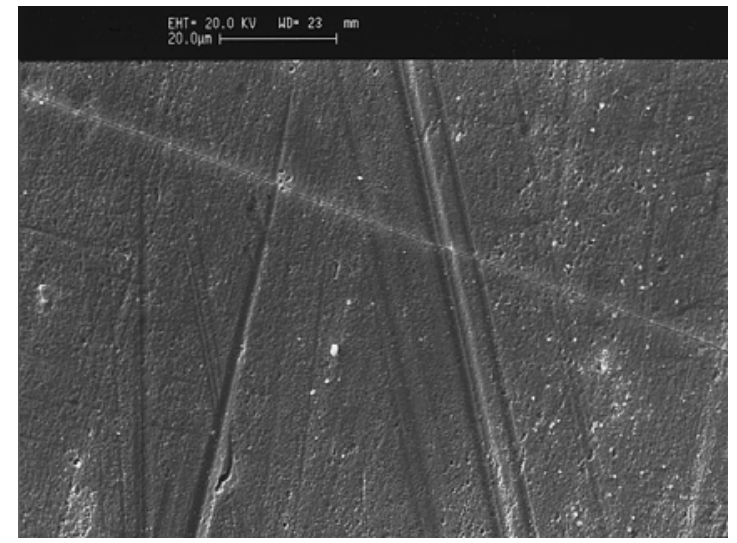

(a)

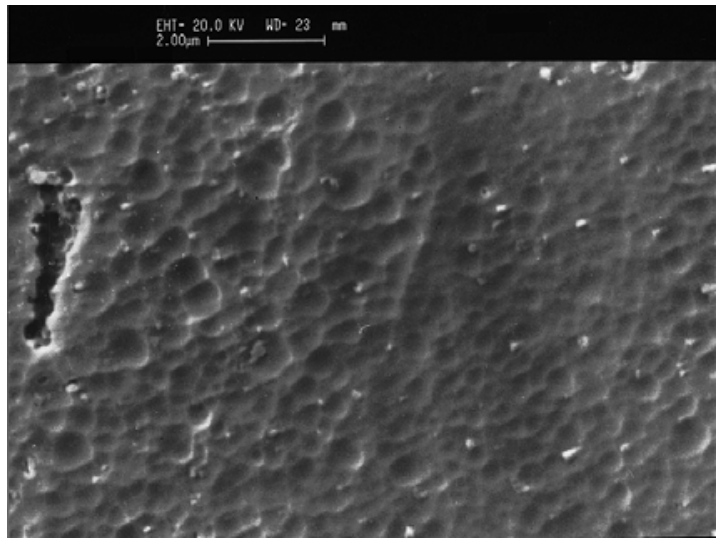

(b)

Figure 16 - Low (a) and high (b) magnification SEM image of BSAA processed 2024T3 clad alloy surface

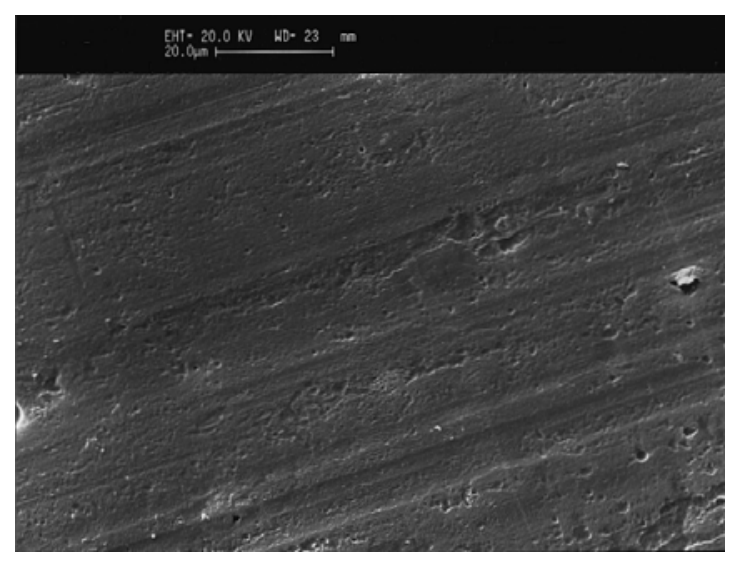

(a)

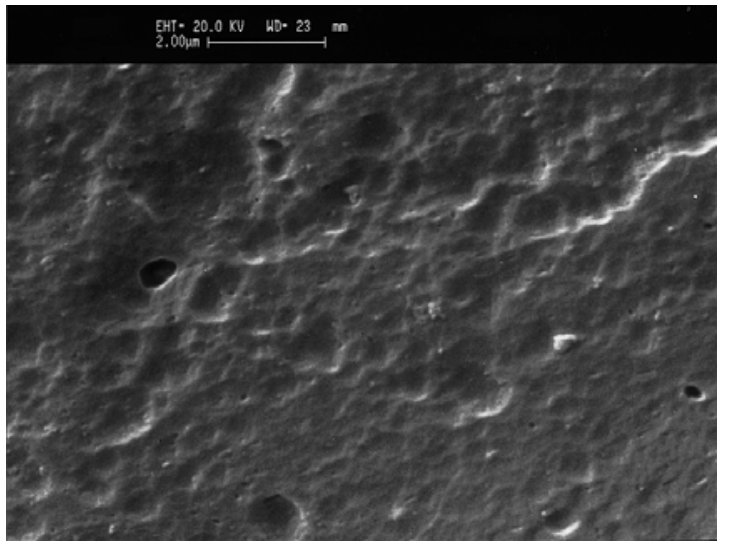

(b)

Figure 17 - Low (a) and high (b) magnification SEM image of BSAA processed 2024T3 bare alloy surface

Stylus profilometry was used to determine the macro-roughness of the anodised substrates; see Table 8 . The finite size of the stylus tip, typically $2 \mu \mathrm{m}$ in diameter, prevents interrogation of the fine pores present on the oxidised surfaces.

\begin{tabular}{|l|l|l|l|}
\hline Treatment & Direction & $\mathrm{R}_{\mathrm{a}}\left(\mathrm{x} \mathrm{10}^{-6} \mathrm{~m}\right)$ & $\mathrm{R}_{\mathrm{t}}\left(\mathrm{x} \mathrm{10}^{-6} \mathrm{~m}\right)$ \\
\hline CAA & $\mathrm{T}$ & 0.061 & 0.536 \\
\hline & $\mathrm{L}$ & 0.055 & 0.451 \\
\hline BSAA & $\mathrm{T}$ & 0.053 & 0.465 \\
\hline & $\mathrm{L}$ & 0.077 & 0.567 \\
\hline
\end{tabular}




\section{Table 8 - Profilometry data for anodised 2024 clad alloy surfaces}

High resolution microscopy of the BSAA processed alloys showed that only the 2024T3 clad alloy exhibited the porous structure as expected from theory; see Figure 18a. However, these pores were less easily resolvable than on the CAA surface, appearing slightly 'sealed' in comparison to the open, more well defined pores of the CAA. Pore and cell wall dimensions for the BSAA oxide range from 10 to $15 \mathrm{~nm}$ and 5 to $10 \mathrm{~nm}$ respectively, with pore densities in the order of 5 to $10 \times 10^{10} \mathrm{~cm}^{-2}$.
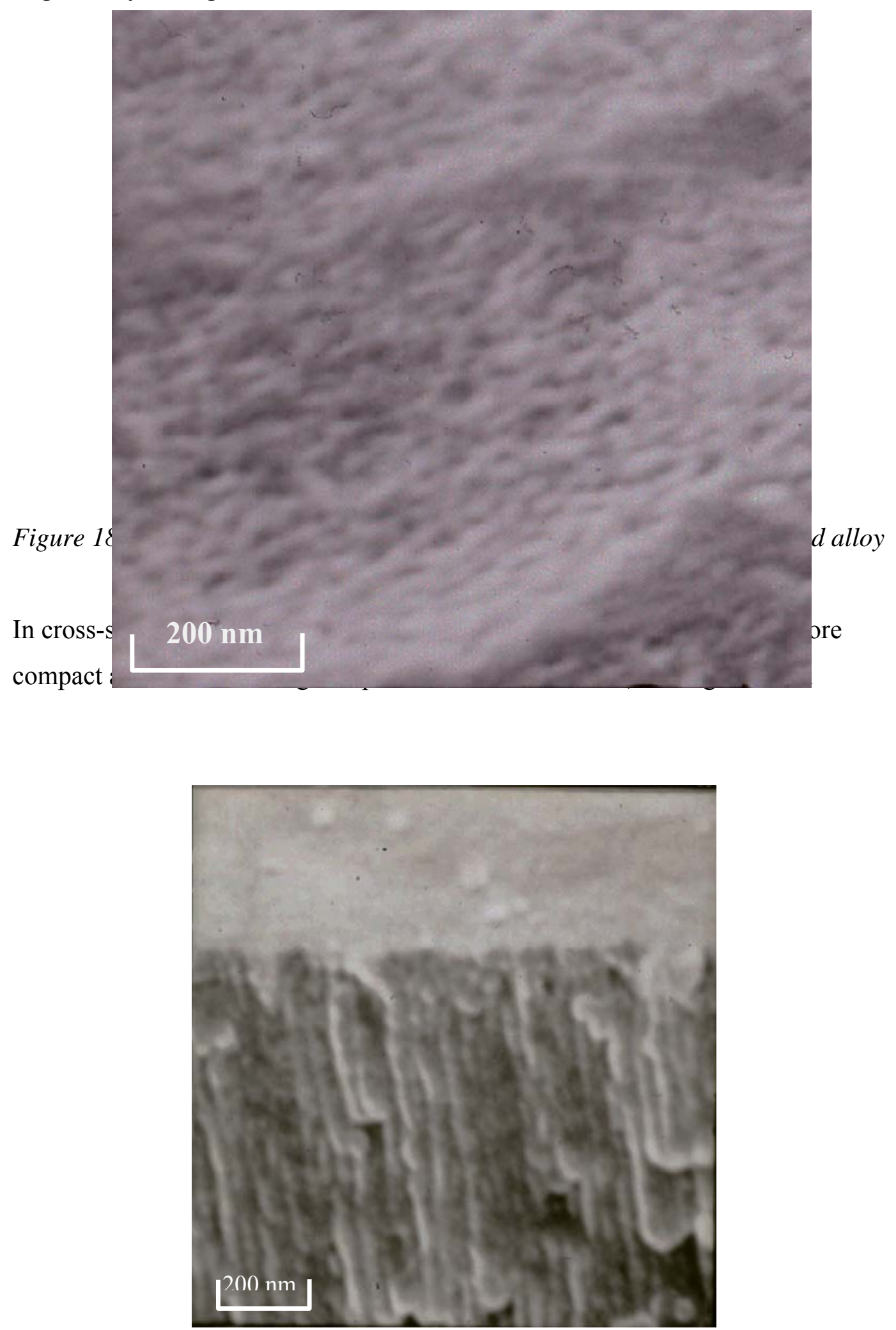
Figure 18b - Cross-sectional image of standard BSAA processed 2024-T3 clad alloy

As with the CAA, the oxide films produced on the bare 2024-T3 by the BSAA process appeared "sponge-like" and less ordered. This is in direct contradiction to the work carried out by Thompson and co-workers for 7075-T6 bare alloy, who reported a columnar branched porous structure. In their work they have made use of electropolishing techniques prior to anodising. This may have removed a lot of the intermetallic particles on the surface that could be responsible for the formation of this "spongy" oxide. Film thicknesses for the BSAA process, acquired from crosssectional images, were $3.5 \pm 0.5 \mu \mathrm{m}$ for $2024-\mathrm{T} 3$ clad alloy and $2.0 \pm 0.5 \mu \mathrm{m}$ for the 2024-T3 bare alloy.

FTIR was carried out to compare the bulk film chemistry after both CAA and BSAA processes. Data from the anodised 2024-T3 bare alloy showed an additional peak for the standard BSAA at approximately 1150 wavenumbers which was absent in the CAA case; Figure 19a. This indicates the oxide contains sulphate with the peak being associated with the strong oxygen-sulphur double bond. The broad peak between 3000 and 3600 wavenumbers is indicative of $\mathrm{H}_{2} \mathrm{O}$ and is very similar for both anodised surfaces.

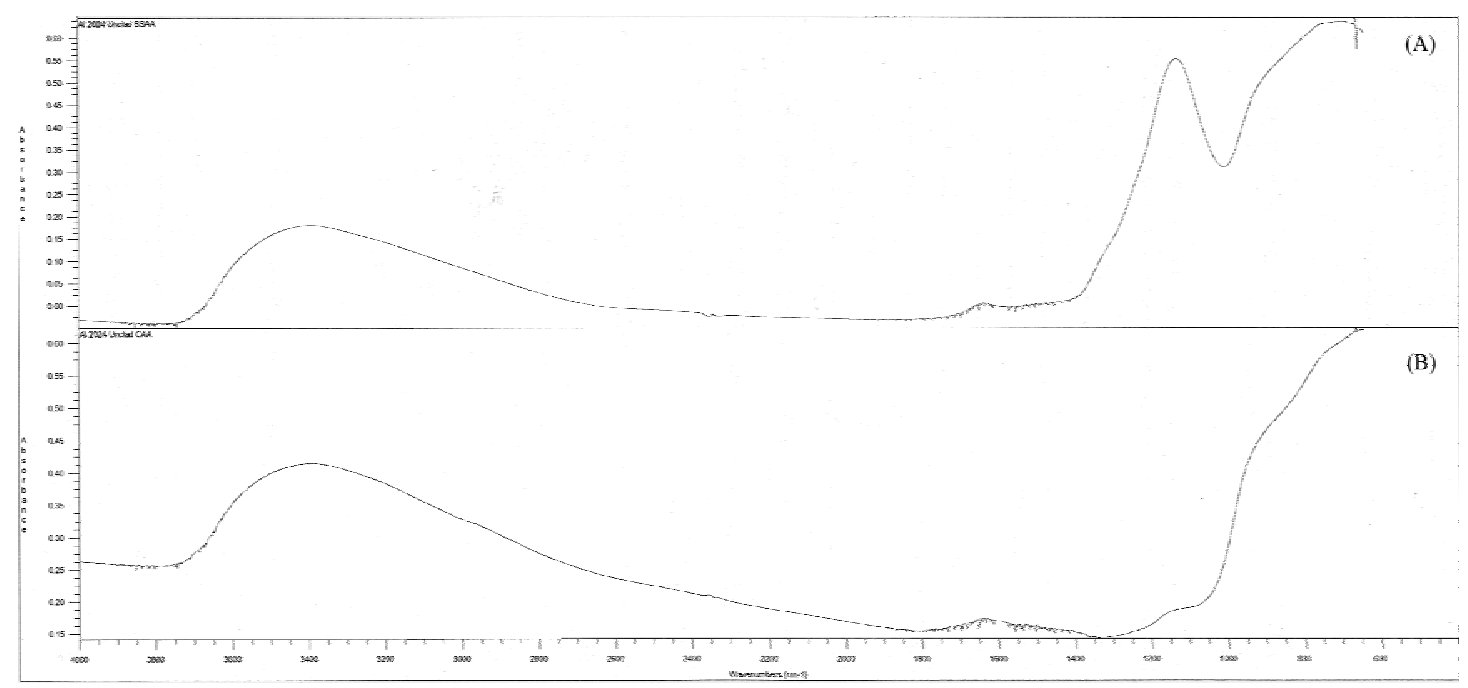


Figure 19a - FTIR scans of BSAA (a) and CAA (b) processed 2024-T3 bare alloy

After heating at $100^{\circ} \mathrm{C}$ the $\mathrm{H}_{2} \mathrm{O}$ peak decreases, as would be expected This can be seen for both the BSAA and CAA anodised 2024-T3 clad surfaces, Figure 19b-c. The broad $\mathrm{H}_{2} \mathrm{O}$ peak may be masking any hydroxyl present on the surface.

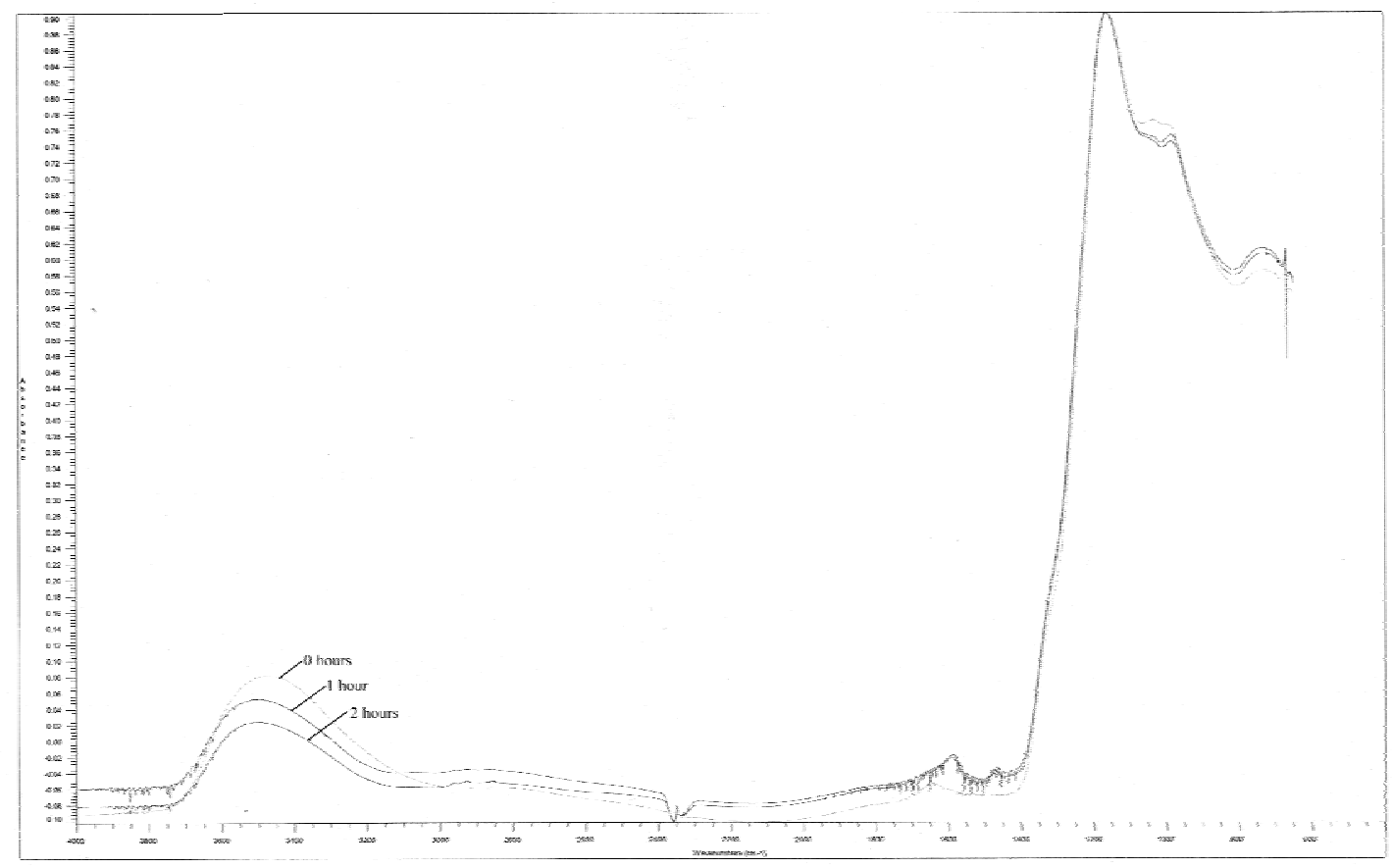

Figure 19b - FTIR scans of BSAA processed 2024-T3 clad alloy heated for various times 


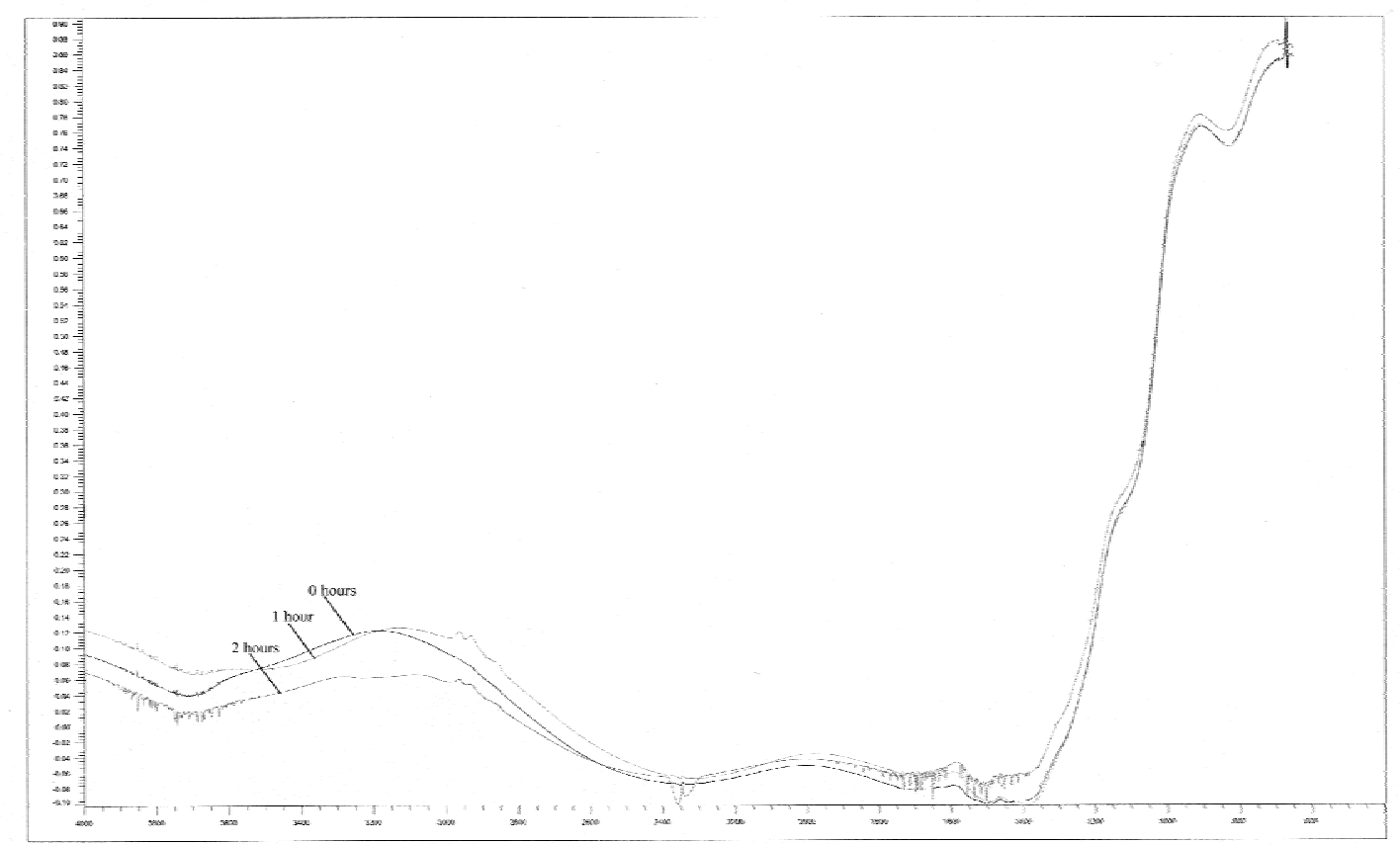

Figure 19c - FTIR scans of CAA processed 2024-T3 clad alloy heated for various times

From all of the above analyses, it appears that the oxides produced by the standard BSAA and CAA processes are very similar in terms of their surface chemistry with evidence for only limited borates and sulphates present within the BSAA oxide. The presence of boron and sulphur at or below the 1 percent level was indicated by XPS. The most significant differences between the oxides produced by the standard processes is the much increased pore size introduced by the CAA process compared with the BSAA. The aim of using a non-standard BSAA is to create the open structure of the CAA with the benign BSAA electrolyte.

\subsubsection{Non-standard anodising parameters}

The significant variables, namely; deoxidising, anodising parameters and post treatment have been studied. The majority of studies consider treatments on the 2024T3 clad alloy only.

\subsubsection{Deoxidising}

Considering the SEM data obtained from the deoxidised surfaces, the Pyrene 14-73, Chemcid 2218 and the EPAD only were considered worthy of further study. SEM showed the surface topography produced by both the Pyrene 14-73 and the Chemcid 
2218 processes is reflected in the subsequently BSAA anodised surface. In crosssection, the structure and thickness of the BSAA oxide following Pyrene 14-73 and Chemcid 2218 pretreatments appears similar to that of the standard BSAA process using the Tri-acid etch, see Figure 20a-b.

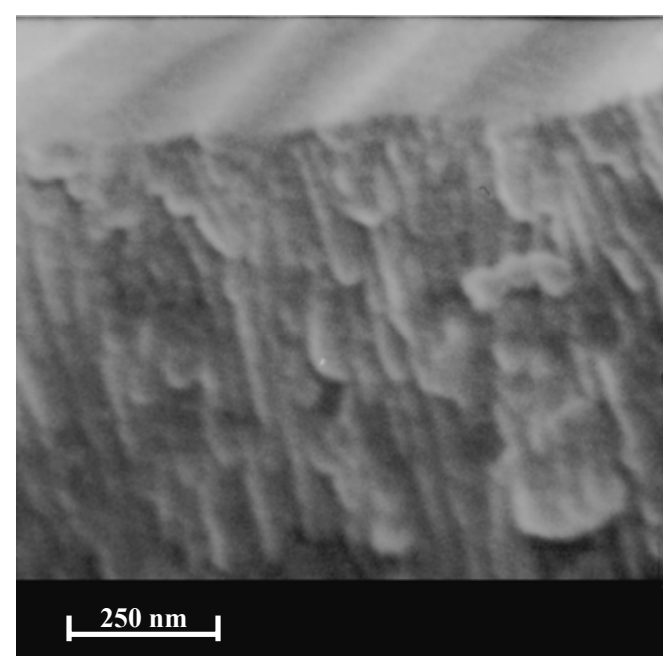

(a)

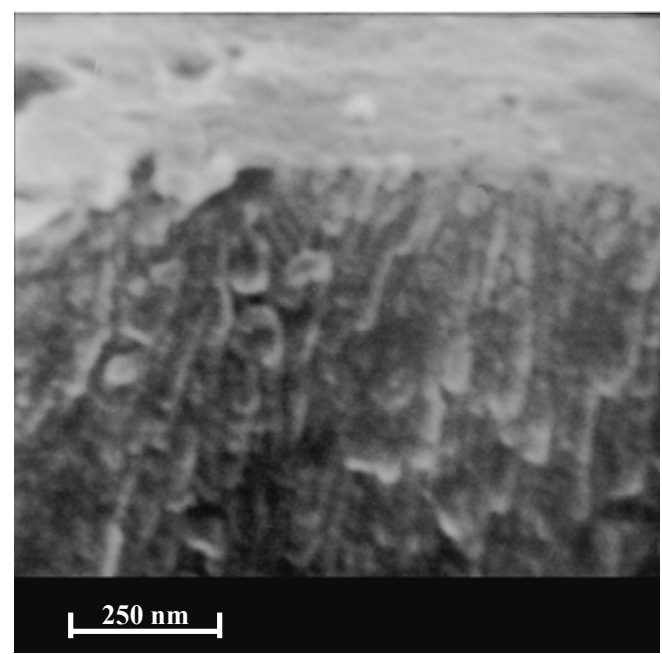

(b)

Figure 20 - Cross-sectional images of Pyrene 14-73 (a) and Chemcid 2218 (b) deoxidised and BSAA processed 2024-T3 clad alloy

SEM images of the EPAD and subsequently BSAA anodised surface are given in Figure 21a-b. These shows the microscopic surface topography of the EPAD is also transferred from the deoxidised surface onto the final anodised film. In cross-section a duplex oxide can clearly be seen. The uppermost section (200 - 300nm of the oxide) consisting of the aforementioned nodules, with some branching taking place. The lower section is more characteristic of the BSAA cross-sections with a columnar oxide visible. 


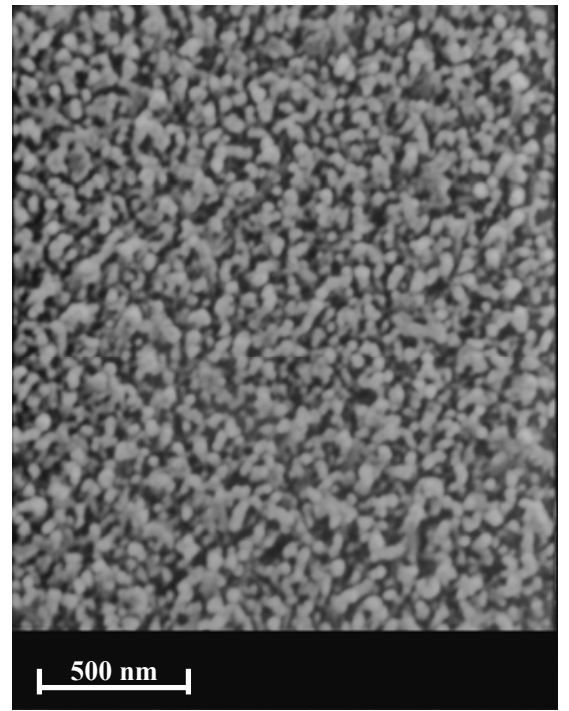

(a)

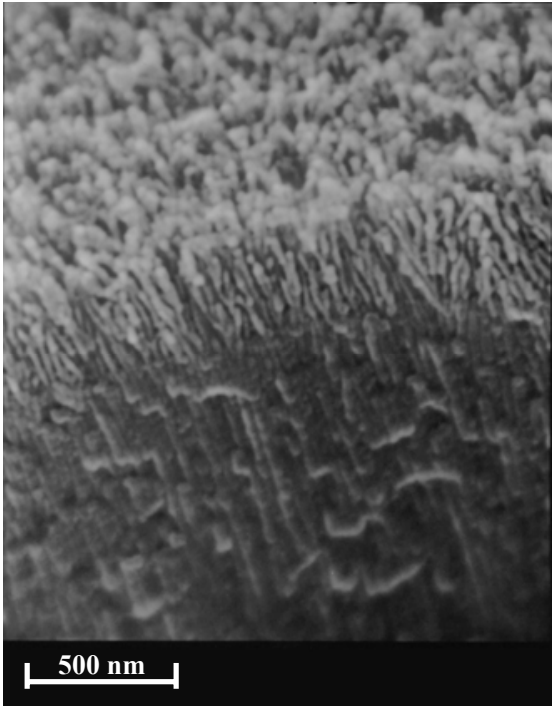

(b)

Figure 21 - Surface topography (a) and cross-section (b) of phosphoric acid deoxidised and BSAA treated 2024-T3 clad alloy

\subsubsection{Anodising electrolyte acid concentration}

Varying the boric and sulphuric acid concentrations gave no increase in porosity compared to the standard BSAA. From Figure 22, which shows the BSAA film thickness as a function of acid concentrations, determined from Eddy current measurements, it is clear that the sulphuric acid concentration does, however, affect the thickness of the anodic oxide. Note that the thickness of the oxide could be important in terms of both corrosion resistance and performance in terms of bare metal fatigue. 


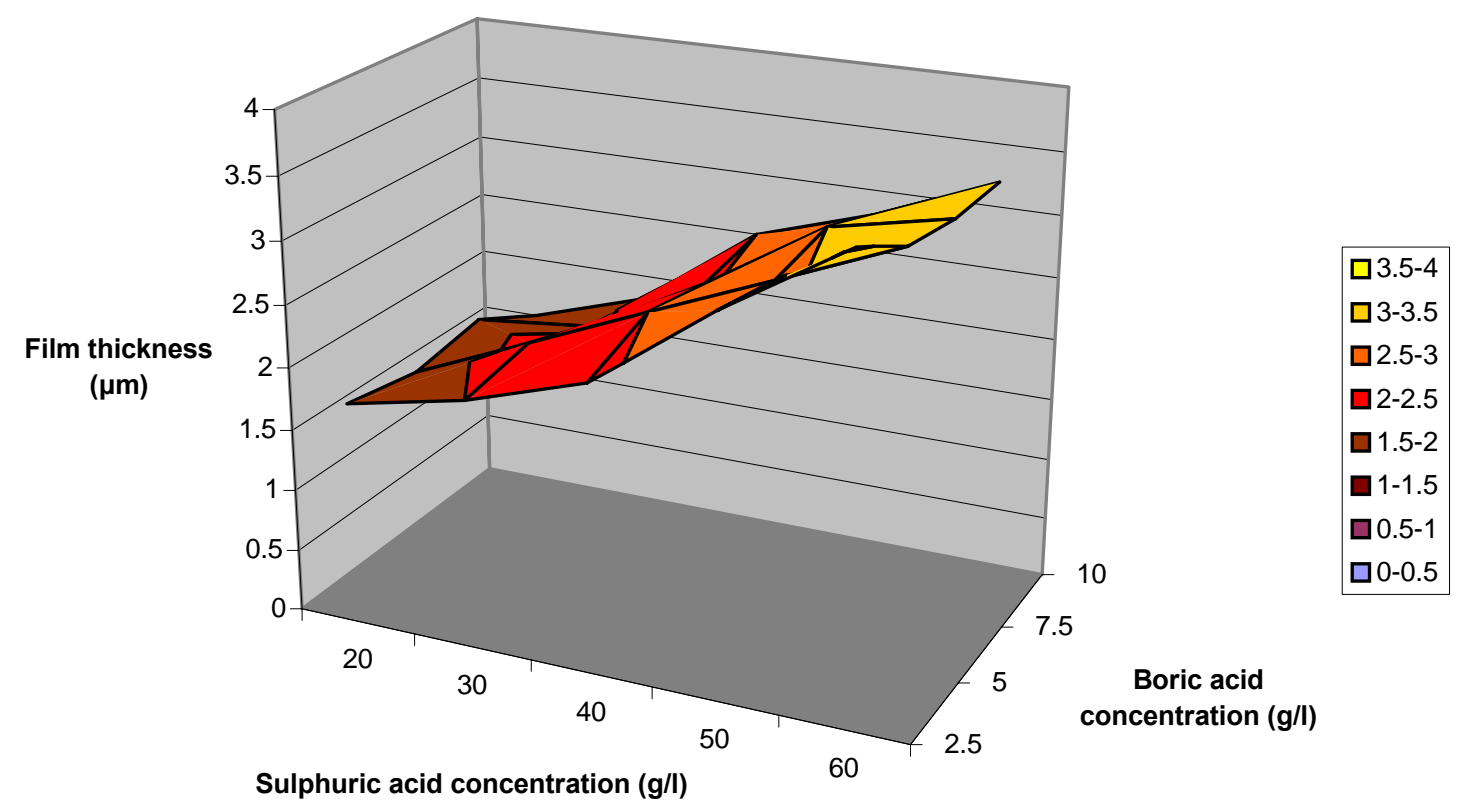

Figure 22 - To show anodic film thicknesess as a function of varying boric and sulphuric acid concentrations in the BSAA electrolyte.

\subsubsection{Anodising electrolyte temperature}

BSAA electrolyte temperatures of $15,20,30,35$, and $40^{\circ} \mathrm{C}$ were studied with standard solution concentrations. The reduced electrolyte temperature of 15 and $20^{\circ} \mathrm{C}$ showed little or no change in pore structure compared to the standard BSAA process operated at $22^{\circ} \mathrm{C}$. As expected, the higher electrolyte temperatures of 30,35 and $40^{\circ} \mathrm{C}$ did show evidence of increasing pore size. However, it was observed that if temperatures were increased greater than $40^{\circ} \mathrm{C}$, then "burning" occurred, rendering the material useless for adhesive bonding due to film breakdown.

From SEM analysis, the electrolyte temperature producing a structure most comparable to that of the CAA oxide was at $35^{\circ} \mathrm{C}$, with pore and cell wall dimensions in the range of 15 to $25 \mathrm{~nm}$ and 10 to $15 \mathrm{~nm}$ respectively; see Figure 23 . Consequently, a full comparative study utilising the $35^{\circ} \mathrm{C}$ or high temperature BSAA (HTBSAA) process was carried out. 


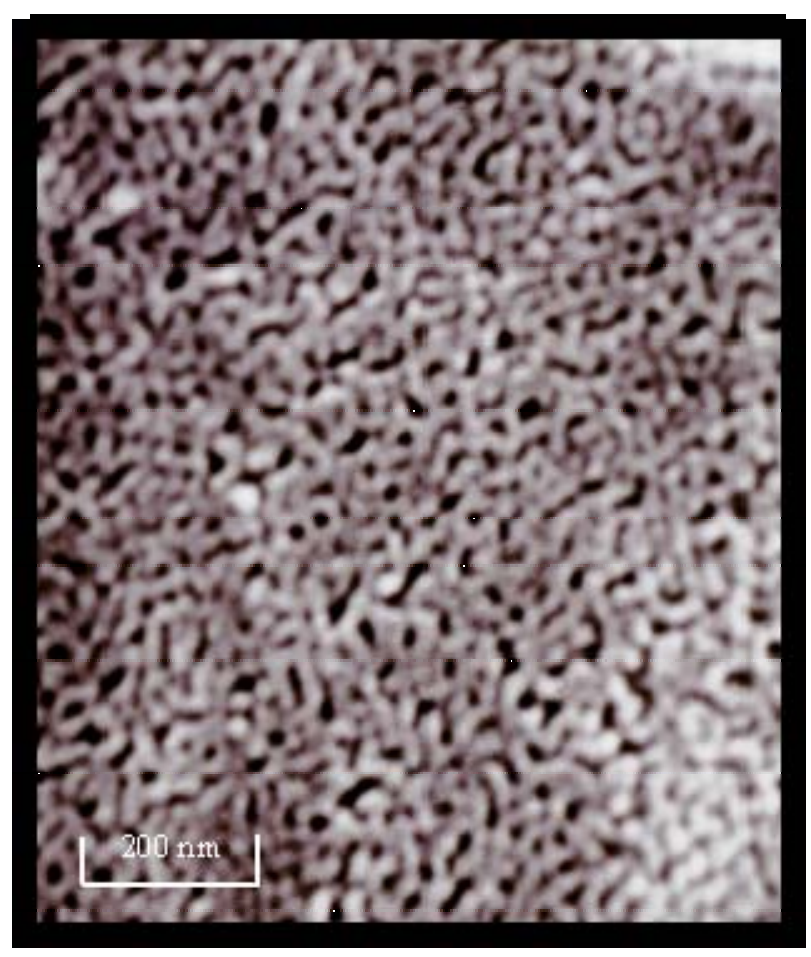

Figure 23 - Surface topography of HTBSAA processed 2024-T3 clad alloy; cf. Figure $18 a$

\subsubsection{Anodising potential}

BSAA anodising potentials was varied from the standard 15 to $25 \mathrm{~V}$, further increases resulted in burning. However, both plan view and cross-sectional images of films anodised at $20 \mathrm{~V}$ and $25 \mathrm{~V}$ showed no increase in porosity with voltage, this was as expected; see Figures 24a-b.

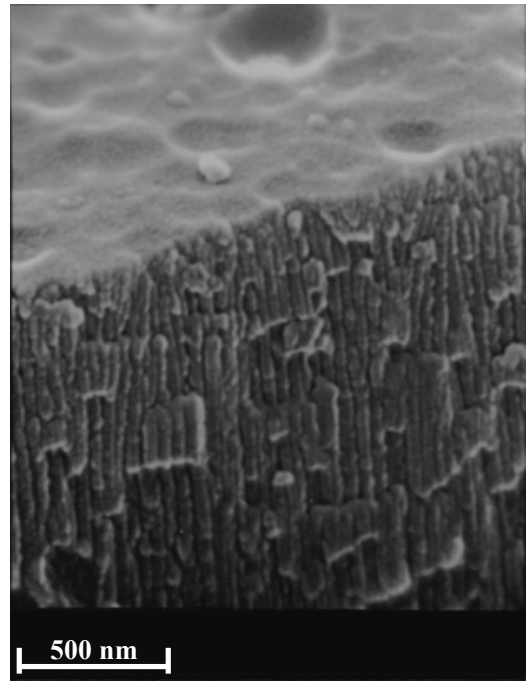

(a)

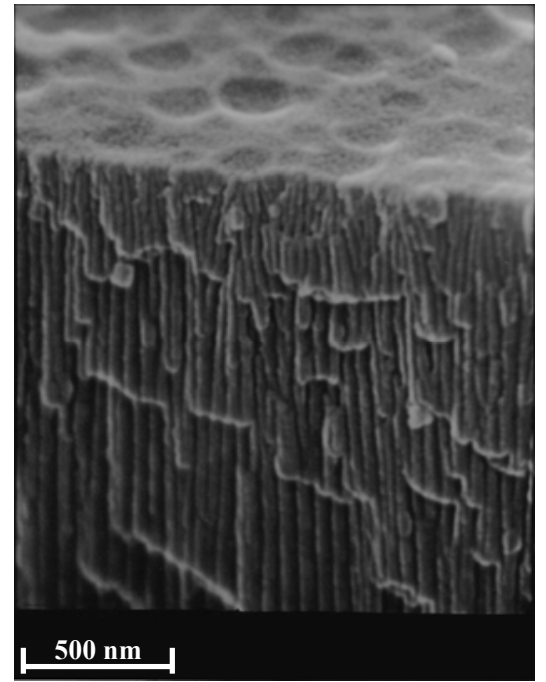

(b)

Figure 24 - Cross-sectional images of BSAA processed 2024-T3 clad alloy with anodising potentials of $20 \mathrm{~V}(\mathrm{a})$ and $25 \mathrm{~V}$ (b) 
The higher anodising potentials for the BSAA process do seem to produce a straighter, more columnar oxide than the standard or HT process, possibly as a result of the increased electric field.

\subsubsection{Post treatment}

Figure 25 shows a standard BSAA surface in plan view following a subsequent PAD treatment. The effect of the dip has been to produce a highly etched surface, no longer uniform or continuous. At higher magnification it can be seen that the dip has significantly altered the surface structure of the anodic oxide, giving a more "feathered" appearance. This may have two effects on the bond durability of a joint: Firstly, the increased surface texture may improve mechanical interlocking and so aid bonding, and; secondly the heavily etched surface may have an adverse effect on the corrosion performance, providing easier access for moisture to access the underlying alloy. Published studies into the effects of phosphoric acid dipping following CAA, SAA and PAA have all concluded that improved results for adhesive bonding can be achieved, so it could be speculated that this surface treatment may likewise prove successful for the BSAA process, if the optimised treatment times are achieved.

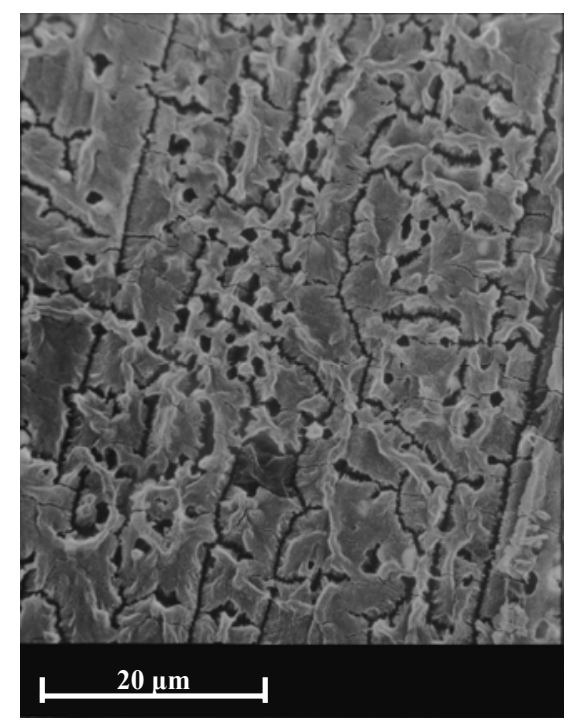

(a)

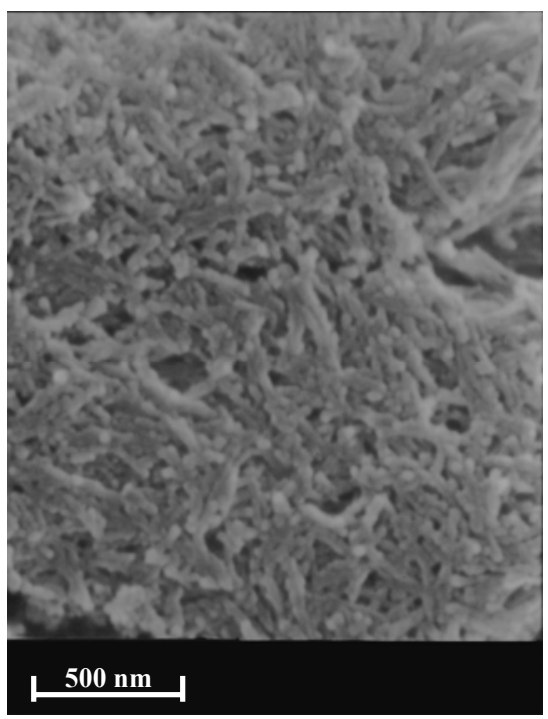

(b)

Figure 25 - Low (a) and high (b) magnifications of the surface topography of BSAA processed 2024-T3 clad alloy after subsequent PAD 


\subsection{Mechanical Testing}

In Section3.1 results have been presented showing the effects of varying parameters within the BSAA process in on the surface morphology and oxide structure in an attempt to produce an oxide analogous to that on CAA treated aluminium alloy. In order to evaluate the significance of these variations a series of mechanical tests were carried out, using the degreased-only and CAA process as benchmarks, the results follow.

\subsubsection{Initial assessment}

To assess initial bond strength and durability produced by the standard CAA and BSAA processes, preliminary joint testing was carried out with the use of SLS and standard wedge test joints. Maximum SLS loads to failure are shown in Table 9, typical standard deviations were $\pm 200 \mathrm{~N}$.

\begin{tabular}{|l|l|}
\hline Surface treatment & Force (N) \\
\hline Degreased-only & 3350 \\
\hline Degreased + Grit-blasted & 5600 \\
\hline Degreased + 'optimised' FPL etched & 7250 \\
\hline Degreased + Tri-acid etched & 7300 \\
\hline Degreased + 'optimised' FPL etched + BSAA & 7700 \\
\hline Degreased + Tri-acid etched + BSAA & 8050 \\
\hline Degreased + 'optimised' FPL etched + CAA 40/50V & 7950 \\
\hline
\end{tabular}

Table 9 - Summary of SLS joint strengths for various surface treatments

These data illustrate trends which are in agreement with many other studies. In particular, the mechanical treatments perform worst of all followed by the chemical and the electrochemical performing best. Note that, in this test, the BSAA process demonstrated excellent initial levels of adhesion with both FPL and Tri-acid deoxidisers. Wedge test data from joints utilising clad 2024-T3 alloy and standard CAA and BSAA processes are presented in Figure 26. It is clear from the wedge test 
data that the crack extensions are much greater using the stanadard BSAA than with the CAA. In simple terms, this indicates that the adhesion performance of joints utilising the standard BSAA process is significantly inferior to those made with the CAA process. As such, this indicates that the BSAA would be an unsuitable drop-in replacement to the CAA process prior to structural bonding.

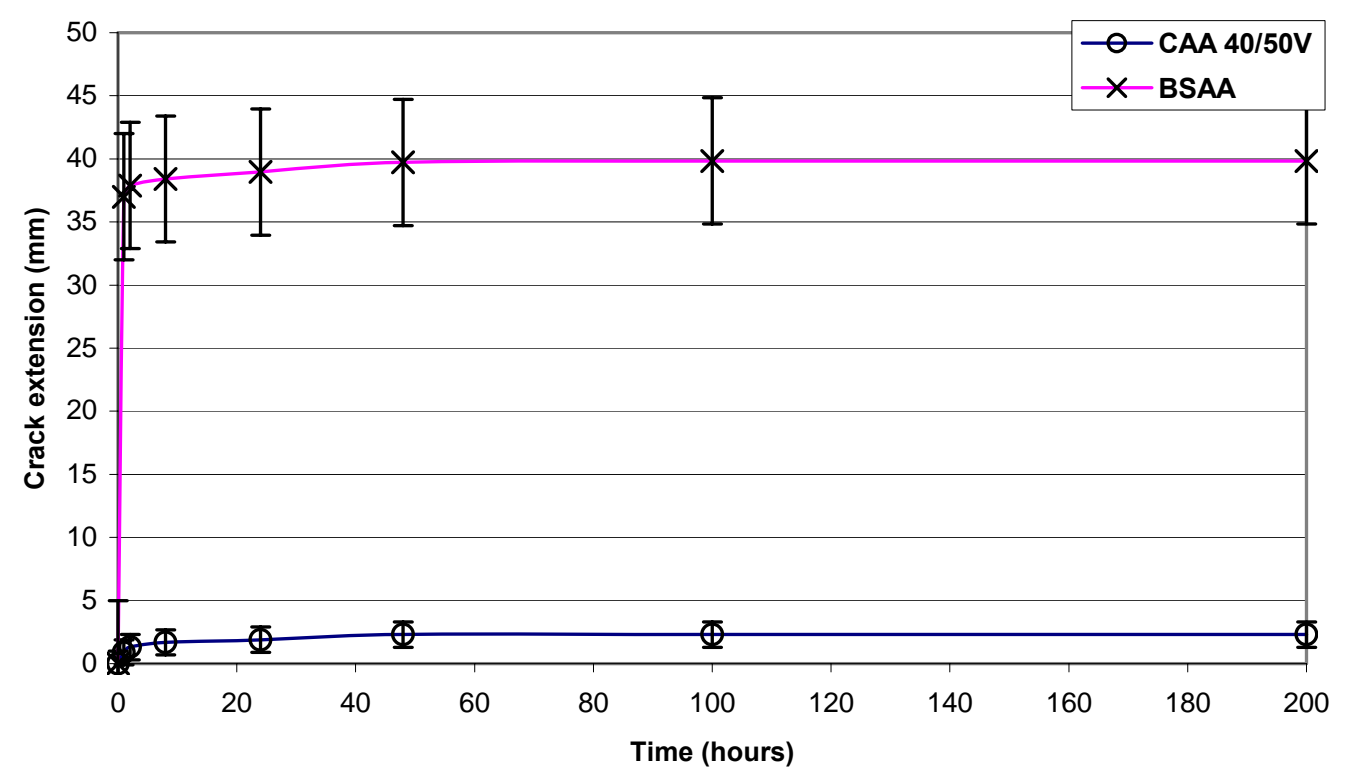

Figure 26 - Summary of wedge test results for standard CAA and BSAA processes on clad 2024 alloy under $60^{\circ} \mathrm{C}$ and $100 \%$ RH conditions

Subsequent analysis of the fracture surfaces generated by the wedge tests showed the CAA joints to clearly fail cohesively within the adhesive. The standard BSAA specimens revealed three visible failure modes over the disbonded area, which was further investigated with the use of XPS. Failure areas were labelled, areas 1 to 3 with both sides of the specimen being analysed, results are presented in Table 10. 


\begin{tabular}{|c|c|c|c|c|c|c|}
\hline Element (At \%) & $\mathbf{C}$ & Al & $\mathbf{N}$ & $\mathbf{O}$ & $\mathrm{Cr}$ & $\mathbf{S i}$ \\
\hline \multicolumn{7}{|l|}{ Area } \\
\hline 1 - Side A & 63.5 & 8.2 & 2.8 & 24.5 & 1.0 & 0.0 \\
\hline 1 - Side B & 73.8 & 0.0 & 5.2 & 19.8 & 0.0 & 1.1 \\
\hline 2 - Side A & 11.8 & 31.9 & 0.0 & 56.3 & 0.0 & 0.0 \\
\hline 2 - Side B & 13.1 & 31.0 & 0.0 & 55.9 & 0.0 & 0.0 \\
\hline 3 - Side A & 79.6 & 0.0 & 2.2 & 17.6 & 0.5 & 0.0 \\
\hline 3 - Side B & 79.8 & 0.0 & 2.7 & 17.5 & 0.0 & 0.0 \\
\hline
\end{tabular}

Table 10 - Summary of XPS data for visibly different failure modes from wedge test specimens for the standard BSAA process

Area 1 showed a mixed cohesive failure at the primer/adhesive interface plus some failure at the oxide interface. Area 2 showed predominantly cohesive failure within the oxide layer and for Area 3, cohesive failure within the adhesive is observed. XPS also confirmed that the CAA treated specimens cohesive failure within the adhesive, the results of which are omitted as similar values for the failure in area 3 of the BSAA treated joint were apparent.

\subsubsection{SLS testing of alternative BSAA processing parameters}

\subsubsection{Variable electrolyte concentrations}

Table 11 shows SLS results from joints prepared after varying the concentrations of boric and sulphuric acids in the electrolyte. This experiment was repeated using the 
'optimised' FPL rather than the standard tri-acid etch treatment with very similar results. As can be seen from the table, no detectable change, within experimental errors, is observed with similarly good values in all cases.

\begin{tabular}{|l|l|l|l|l|l|}
\hline \multicolumn{1}{|r|}{$\mathrm{H}_{2} \mathrm{SO}_{4}$} & $20 \mathrm{~g} / 1$ & $30 \mathrm{~g} / 1$ & $40 \mathrm{~g} / 1$ & $50 \mathrm{~g} / 1$ & $60 \mathrm{~g} / 1$ \\
\hline $2.5 \mathrm{~g} / 1$ & $7950 \mathrm{~N}$ & $8200 \mathrm{~N}$ & $8000 \mathrm{~N}$ & $8150 \mathrm{~N}$ & $7950 \mathrm{~N}$ \\
\hline $5 \mathrm{~g} / 1$ & $7850 \mathrm{~N}$ & $8100 \mathrm{~N}$ & $8050 \mathrm{~N}$ & $7950 \mathrm{~N}$ & $7800 \mathrm{~N}$ \\
\hline $7.5 \mathrm{~g} / 1$ & $7800 \mathrm{~N}$ & $8000 \mathrm{~N}$ & $7750 \mathrm{~N}$ & $8350 \mathrm{~N}$ & $7900 \mathrm{~N}$ \\
\hline $10 \mathrm{~g} / 1$ & $7750 \mathrm{~N}$ & $7850 \mathrm{~N}$ & $8100 \mathrm{~N}$ & $8400 \mathrm{~N}$ & $7950 \mathrm{~N}$ \\
\hline
\end{tabular}

Table 11 - Summary of initial joint strengths of SLS joints for varying concentrations of electrolyte in BSAA in conjunction with Tri-acid etch.

Further studies were carried out using SLS joints which were exposed to elevated temperature and humidity for 30 days in a 5\% sodium chloride solution. The extremes of concentration and standard solution make-up were utilised and measurements were recorded of residual joint strength after exposure. Again, this resulted in no distinguishable variations between dissimilar anodising parameters; see Table 12.

\begin{tabular}{|l|l|l|}
\hline BSAA electrolyte concentration & $\begin{array}{l}\text { Unexposed joint } \\
\text { strength }(\mathrm{N})\end{array}$ & $\begin{array}{l}\text { Residual joint strength } \\
\text { after exposure }(\mathrm{N})\end{array}$ \\
\hline $20 \mathrm{~g} / 1 \mathrm{H}_{2} \mathrm{SO}_{4}, 2.5 \mathrm{~g} / 1 \mathrm{H}_{3} \mathrm{BO}_{3}$ & 7950 & 7650 \\
\hline $20 \mathrm{~g} / 1 \mathrm{H}_{2} \mathrm{SO}_{4}, 10 \mathrm{~g} / \mathrm{l} \mathrm{H}_{3} \mathrm{BO}_{3}$ & 7750 & 7750 \\
\hline $60 \mathrm{~g} / 1 \mathrm{H}_{2} \mathrm{SO}_{4}, 2.5 \mathrm{~g} / 1 \mathrm{H}_{3} \mathrm{BO}_{3}$ & 7950 & 8150 \\
\hline $40 \mathrm{~g} / 1 \mathrm{H}_{2} \mathrm{SO}_{4}, 7.5 \mathrm{~g} / 1 \mathrm{H}_{3} \mathrm{BO}_{3}$ & 7750 & 7750 \\
\hline $60 \mathrm{~g} / 1 \mathrm{H}_{2} \mathrm{SO}_{4}, 10 \mathrm{~g} / 1 \mathrm{H}_{3} \mathrm{BO}_{3}$ & 7950 & 8050 \\
\hline $\mathrm{CAA} 40 / 50 \mathrm{~V}$ & 7950 & 7850 \\
\hline
\end{tabular}

Table 12 - Summary of joint strengths for varying BSAA concentrations of electrolyte before and after exposure to $5 \% \mathrm{NaCl}$ solution for 30 days

\subsubsection{The influence of increased temperature}


Table 13 shows the results obtained from varying the anodising bath temperatures with the standard BSAA electrolyte for SLS joints. Values obtained, once again, do not show any difference, within experimental errors, in joint strengths either between elevated or reduced temperatures.

\begin{tabular}{|l|l|}
\hline Temperature $\left({ }^{\circ} \mathrm{C}\right)$ & Joint Strength $(\mathrm{N})$ \\
\hline 15 & 7950 \\
\hline 26 & 8350 \\
\hline 35 & 7750 \\
\hline 40 & 7800 \\
\hline
\end{tabular}

Table 13 - Summary of initial joint strengths from SLS joints for varying bath temperatures, using $50 \mathrm{~g} / \mathrm{l} \mathrm{H}_{2} \mathrm{SO}_{4}, 7.5 \mathrm{~g} / \mathrm{l} \mathrm{H}_{3} \mathrm{BO}_{3}$

\subsubsection{Wedge testing of alternative BSAA processing parameters}

The preceding SLS test data show the BSAA processing variants providing equivalent joint strengths to the CAA processed joints. At first glance this would be considered a positive result, but as has been demonstrated for the standard BSAA process, which also displayed good initial joint strength using SLS testing, failure under wedge test conditions was proven to be disastrous in comparison to the CAA. The SLS test was therefore not considered to be suitably discriminating between these reasonably wellprepared surfaces. Consequently, wedge testing was also carried out for the full range of BSAA variants.

A graphical representation of the mean crack propagation lengths after 100 hours exposure for all BSAA conditions is show in Figure 27; reduced crack extensions indicate a better performing process. The results here seem to suggest that the pore structure or surface topography of the anodised surface has a major influence on the bond durability. The surface treatments with bond performance comparable to the CAA include: HTBSAA; EPAD combined with the standard BSAA; the standard BSAA with subsequent PAD and finally, though to a lesser degree; the grit-blasted surface treatment combined with the BSAA process. The grit-blast plus standard BSAA treatment was not studied further due to its slightly lower performance in wedge testing. Note that these data could easily be converted to fracture energies to 
provide quantitative pseudo-engineering data. This was not carried out as for this purpose only qualitative comparative data was required.

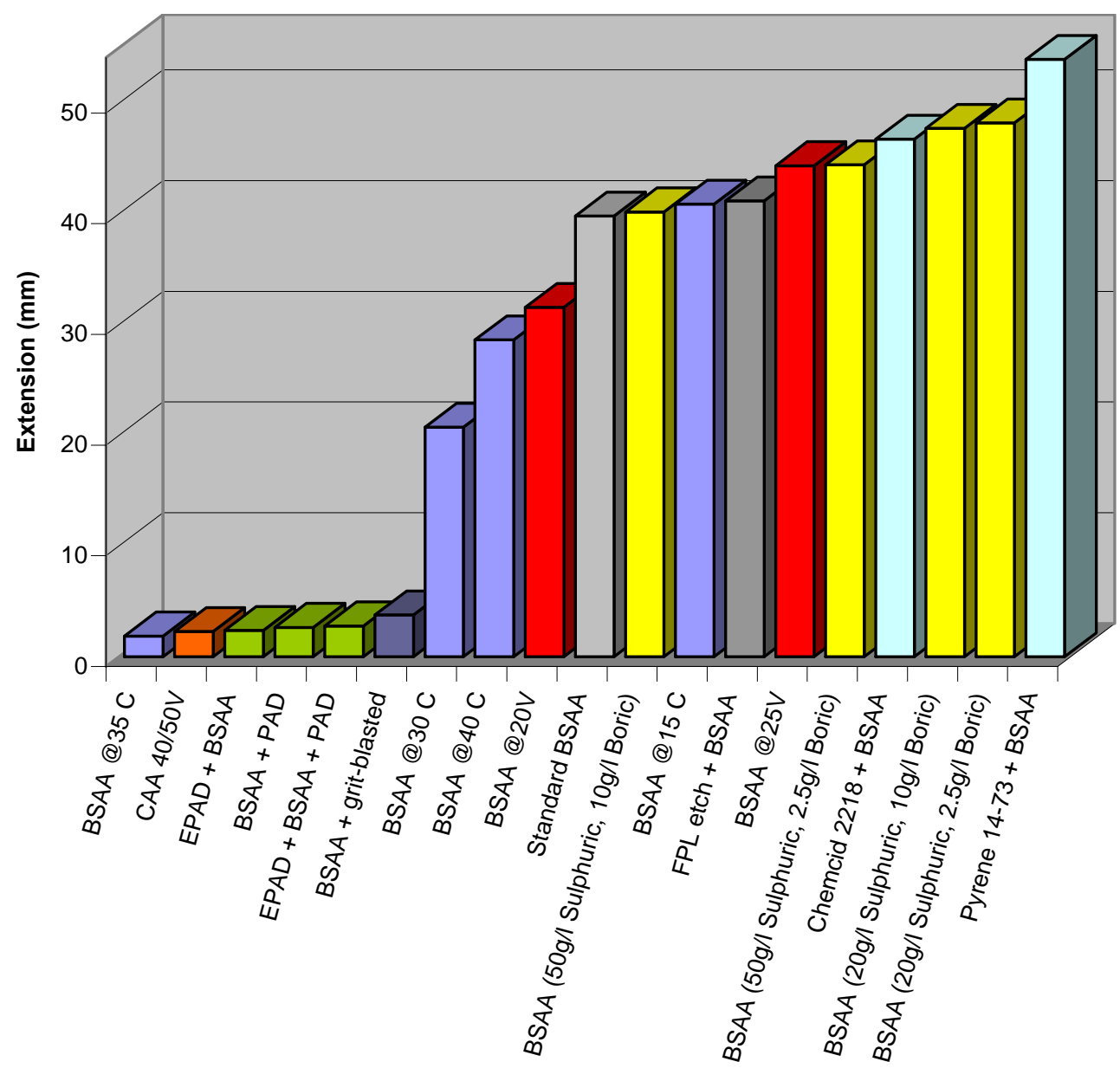

Figure 27 - Summary of mean crack length after 100 hours exposure for various surface treatments on 2024-T3 clad alloy using the wedge test

\subsubsection{Further evaluation of the HTBSAA process}

Figure 28 illustrates the results obtained from the environmentally aged, bare 2024-T3 SLS joints, pretreated using either the CAA or the HT BSAA process. 7075-T6 alloy adherends were also used for comparative purposes to see if these results are alloy sensitive. The results show that there are marginal differences in joint strengths between the HTBSAA and the CAA joints, note that the errors in this experiment were found to be close to $5 \%$. For the unexposed treated specimens the difference in initial joint strengths between the 2024-T3 and the 7075-T6 bare alloys are to be 
expected. The 7075-T6 alloy has a greater stiffness causing less peel, this results in an increase in joint strength with respect to the 2024-T3 bare alloy.

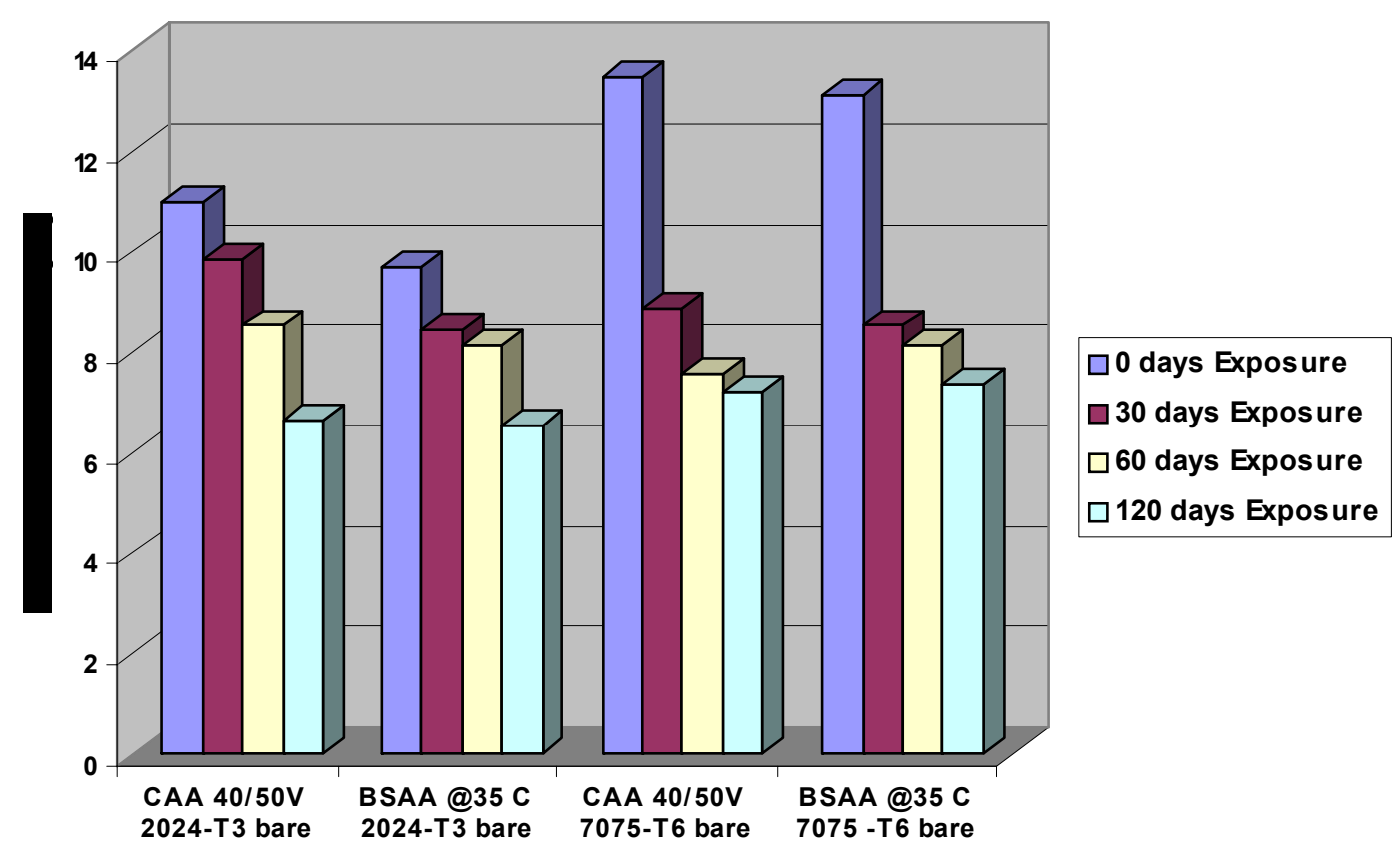

Figure 28 - Summary of environmentally exposed SLS joint strengths

Wedge test data from both the HTBSAA and CAA processes with the 2024-T3 bare and clad alloys in addition to the 7075-T6 bare and clad alloy adherends are presented in Figures 29a-b. These adherends were pretreated in an industrial environment but tested under laboratory conditions. Significantly, in all cases the HTBSAA wedge test joints failed cohesively within the adhesive. In contrast, with the CAA 2024-T3 and 7075-T6 clad specimens, failure appeared to be either cohesive within the adhesive or within the primer layer. The latter failure mechanism was subsequently identified as being caused by excess application of the primer on the CAA surface creating a structurally weak layer. The primer thickness was more carefully controlled, within the manufacturers recommended limits, in all other tests. 


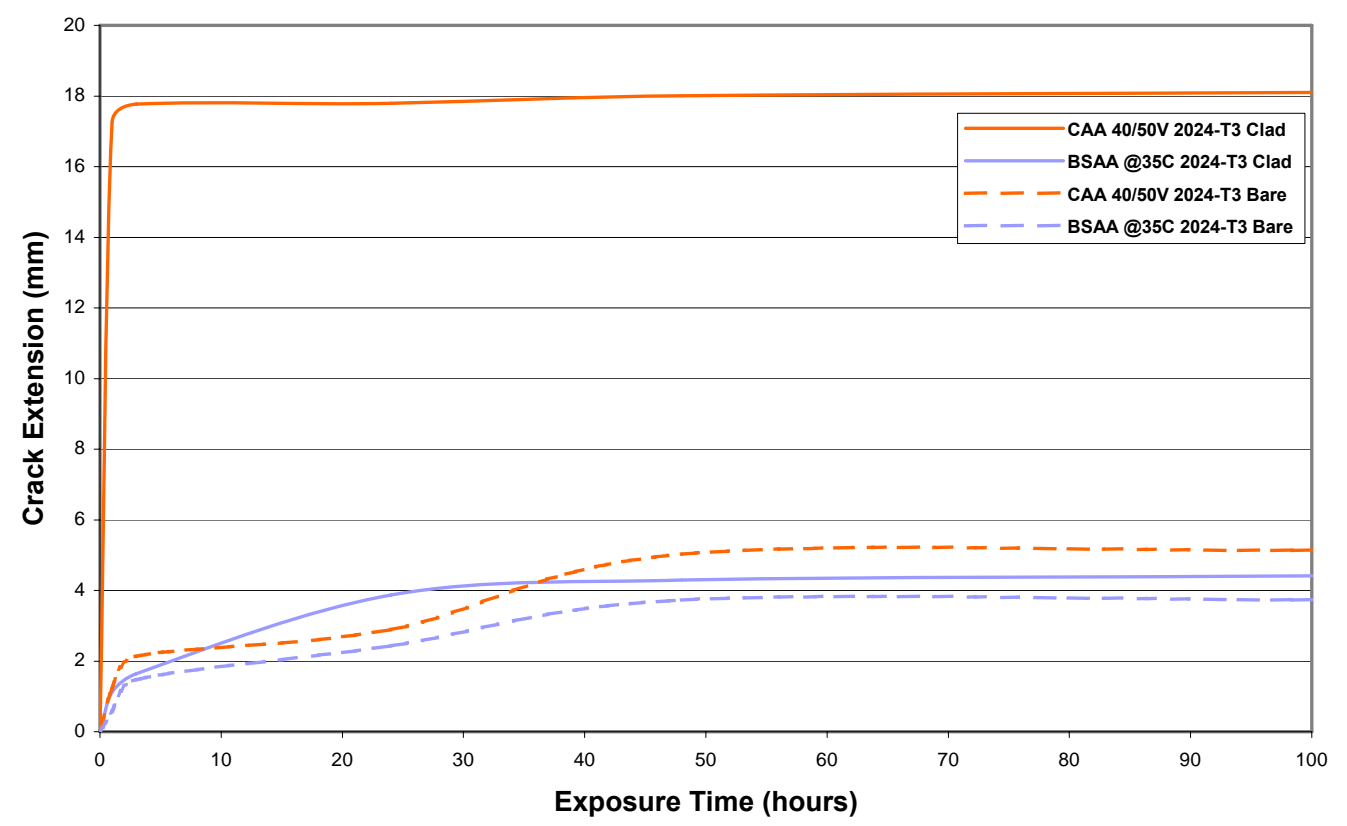

(a)

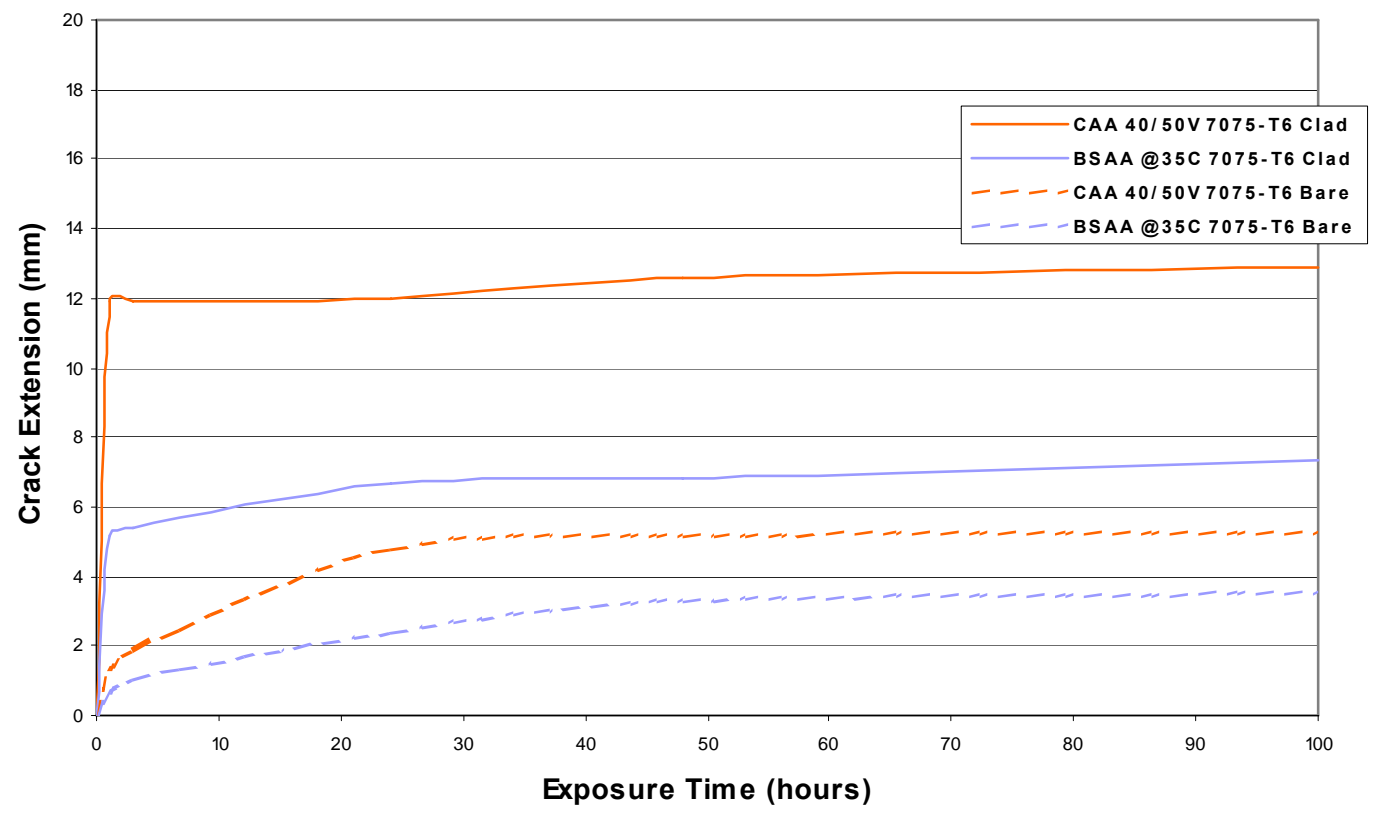

(b)

Figure 29 - Summary of wedge test data for HT BSAA and CAA 40/50V for clad and bare (a) 2024-T3 and (b) 7075-T6 alloys

Peel testing has also been shown to be capable of being highly selective between surface pretreatments. Floating roller peel testing in this work showed favourable results for the 2024-T3 clad alloy substrate, processed using the HTBSAA, compared to that of the CAA peel test results; see Figure 30. In this case the HTBSAA gave 
approximately six times the peel strength per unit width compared to the standard CAA.

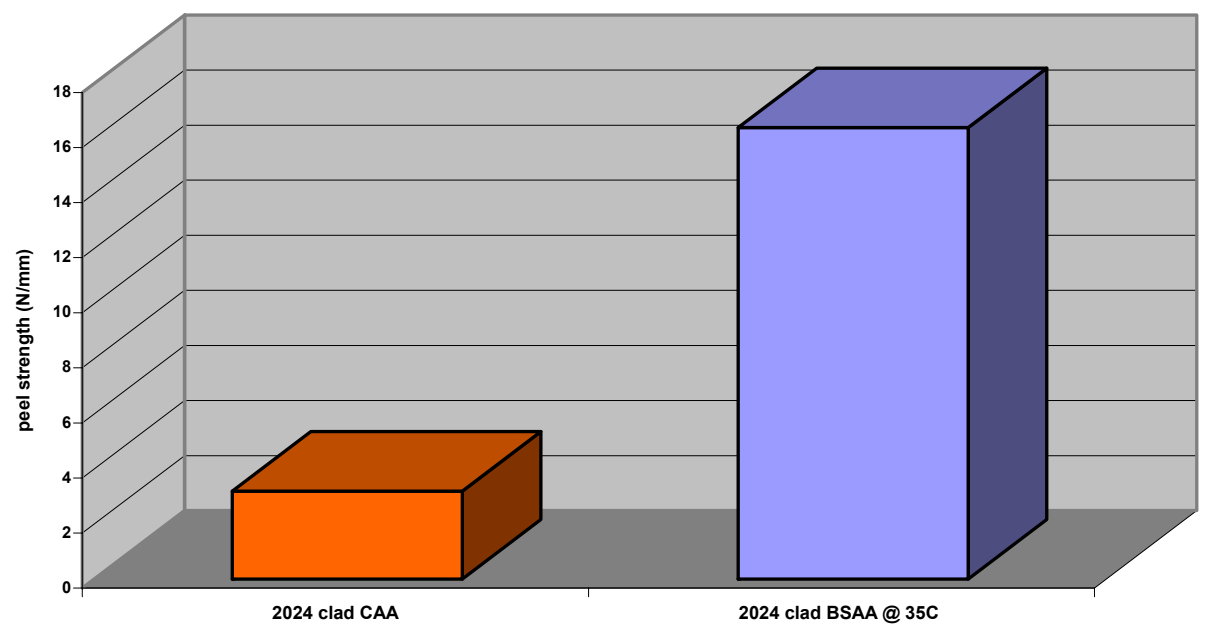

Figure 30 - Summary of peel testing for CAA and HTBSAA processes

Two aspects of fatigue were considered within this study. Firstly, the bare metal fatigue behaviour of the bulk substrates only and secondly the fatigue performance of bonded joints exposed to a sinusoidal load as previously detailed.

Firstly, a possible problem associated with the HTBSAA process is the slightly increased film thickness and as a result its increased coating weights. It has been proposed that as coating weights increase a reduction in fatigue performance occurs, but this may have a positive effect on corrosion resistance, so an optimised coating weight is required. Results to assess fatigue strength loss of the metal adherend are presented in Table 14. These show that 2024-T3 bare alloy dumb-bell specimens anodised with the HTBSAA provide better fatigue performances than that of the CAA processed specimens. This demonstrates that in practice the HTBSAA process does not have any increased detrimental effects on the fatigue properties of the anodised substrate compared with the CAA. 


\begin{tabular}{|l|l|l|}
\hline & CAA 40/50V & BSAA@35 ${ }^{\circ} \mathrm{C}$ \\
\hline $\begin{array}{l}\text { At } 100000 \text { cycles } \\
\text { Reduction of stress }(\%)\end{array}$ & 20 & 18 \\
\hline
\end{tabular}

Table 14 - Summary of fatigue strength loss after HT BSAA and CAA 40/50V processes

Secondly, fatigue data obtained using SLS joints with 2024-T3 clad alloy show a large increase in fatigue performance gained from anodising compared to degreasing or acid etching. The data also suggests that the EPAD combined with the BSAA, performs comparable to or better than the standard BSAA and HT BSAA. Table 15 gives mean cycles-to-failure for joints tested either in air or exposed to DI water immersion.

\begin{tabular}{|l|l|l|}
\hline \multirow{2}{*}{ Surface treatment } & \multicolumn{2}{|l|}{ Maximum Cycles to failure } \\
\cline { 2 - 3 } & Air & Water \\
\hline Degreased only & 2236 & 3387 \\
\hline Tri-acid & 724603 & 409307 \\
\hline FPL & $2358100^{*}$ & $936687^{*}$ \\
\hline Standard BSAA & $1531773^{*}$ & $1928228^{*}$ \\
\hline HT BSAA & $>3380972$ & $1764259^{*}$ \\
\hline PAD + BSAA & $>4310325$ & $>2554557$ \\
\hline CAA 40/50V & $>3276169$ & $3053057^{*}$ \\
\hline
\end{tabular}

Table 15 - Fatigue performance data for various surface treated bonded joints

Note that, in Table 15, * indicates failure within the metal adherend.

\subsection{Corrosion Testing}

From the previous sections the HTBSAA process was identified as capable of producing a similar oxide structure to that of the CAA process. In addition, the mechanical testing carried out has shown that this and other modified BSAA processes are also capable of producing equivalent or better bond strengths and durability to that of the CAA. This section concentrates on a number of corrosion 
studies using both the CAA and HTBSAA processes to assess their relative levels of corrosion resistance.

\subsubsection{Salt spray}

Test results indicated that the corrosion resistance of the HTBSAA to salt spray was slightly inferior to the CAA with the bare 2024-T3. In contrast, the clad HTBSAA treated alloy easily passed this test. The CAA would be expected to "pass" the standard salt spray test for all alloy types.

\subsubsection{Filliform corrosion}

Results from filiform corrosion testing are presented in Table 16. From this data it can be seen that the CAA pretreatment shows a marginal corrosion improvement for the 2024-T3 clad substrate when compared to the HTBSAA pretreatment, both in the vertical and horizontal scratch directions. Taking into consideration the standard deviation for these values it is suggested that this difference is not significant.

\begin{tabular}{|l|l|l|l|l|l|l|}
\hline & \multicolumn{3}{|l|}{ Vertical scratch } & \multicolumn{3}{l|}{ Horizontal scratch } \\
\hline $\begin{array}{l}\text { Substrate \& } \\
\text { Pretreatment }\end{array}$ & $\begin{array}{l}\text { Max. } \\
\text { Length } \\
(\mathrm{mm})\end{array}$ & $\begin{array}{l}\text { Total } \\
\text { Corrosion } \\
\left(\mathrm{mm}^{2}\right)\end{array}$ & $\begin{array}{l}\text { Standard } \\
\text { Deviation }\end{array}$ & $\begin{array}{l}\text { Max. } \\
\text { Length } \\
(\mathrm{mm})\end{array}$ & $\begin{array}{l}\text { Total } \\
\text { Corrosion } \\
\left(\mathrm{mm}^{2}\right)\end{array}$ & $\begin{array}{l}\text { Standard } \\
\text { Deviation }\end{array}$ \\
\hline $\begin{array}{l}\text { 2024-T3 Clad } \\
\text { CAA }\end{array}$ & 1.0 & 9.1 & 2.0 & 1.5 & 14.4 & 4.6 \\
\hline $\begin{array}{l}\text { 2024-T3 Clad HT } \\
\text { BSAA }\end{array}$ & 1.0 & 10.8 & 3.9 & 2.0 & 17.8 & 4.9 \\
\hline $\begin{array}{l}\text { 2024-T3 Bare } \\
\text { CAA }\end{array}$ & 2.0 & 19.4 & 2.4 & 1.5 & 18.7 & 4.8 \\
\hline $\begin{array}{l}\text { 2024-T3 Bare HT } \\
\text { BSAA }\end{array}$ & 1.5 & 11.6 & 2.9 & 1.0 & 13.0 & 2.9 \\
\hline
\end{tabular}

Table 16 - Filiform corrosion data for various anodised substrates

In contrast, the filiform corrosion results for the 2024-T3 bare anodised substrates suggest that the CAA pretreatment offers less corrosion protection than that of the HT 
BSAA pretreatment, in both the vertical and horizontal scratch directions. Again, the standard deviations indicate that this is a marginal rather than a large difference.

\subsubsection{Linear polarisation experiments}

Linear polarisation studies were carried out with various pretreatment options and alloys. The following section provides a summary only of the more significant results.

All plots for the untreated bare and clad 2024-T3 alloys were of a similar nature. The untreated clad alloys show a slight increase in corrosion resistance in comparison to the untreated bare alloys indicated by the decrease in the limiting current on the anodic section of the curves; see Figure 31.

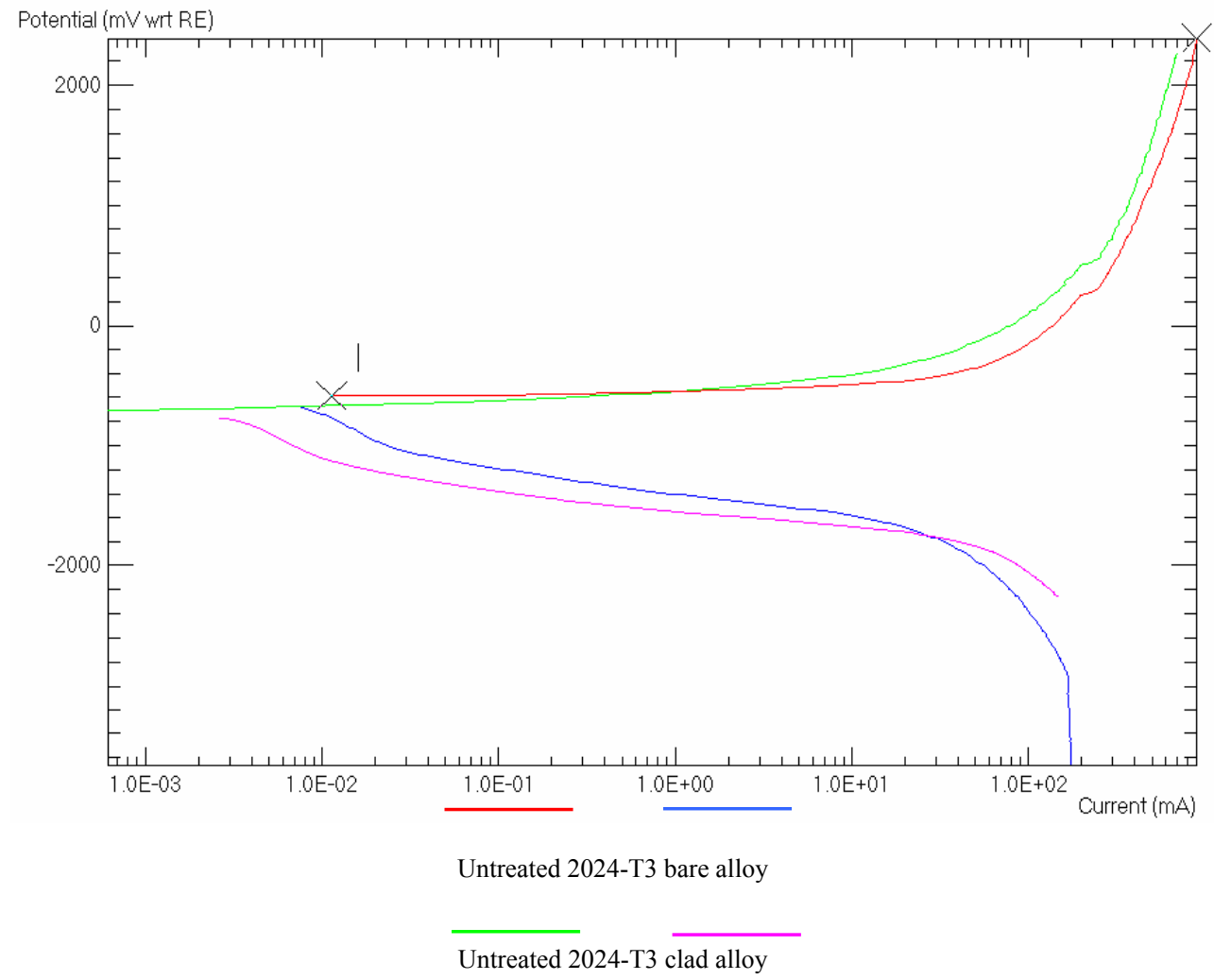

Figure 31 - Linear polarisation curves for untreated 2024-T3 aluminium alloy

A further increase in corrosion resistance is present in the HTBSAA processed 2024T3 alloys, again shown by the decrease in limiting current in the anodic direction; see Figure 32. This is also true when considering cathodic corrosion. The results of 
testing "sealed" anodic films are also presented for comparative purposes. In the interests of brevity these data will not be discussed.

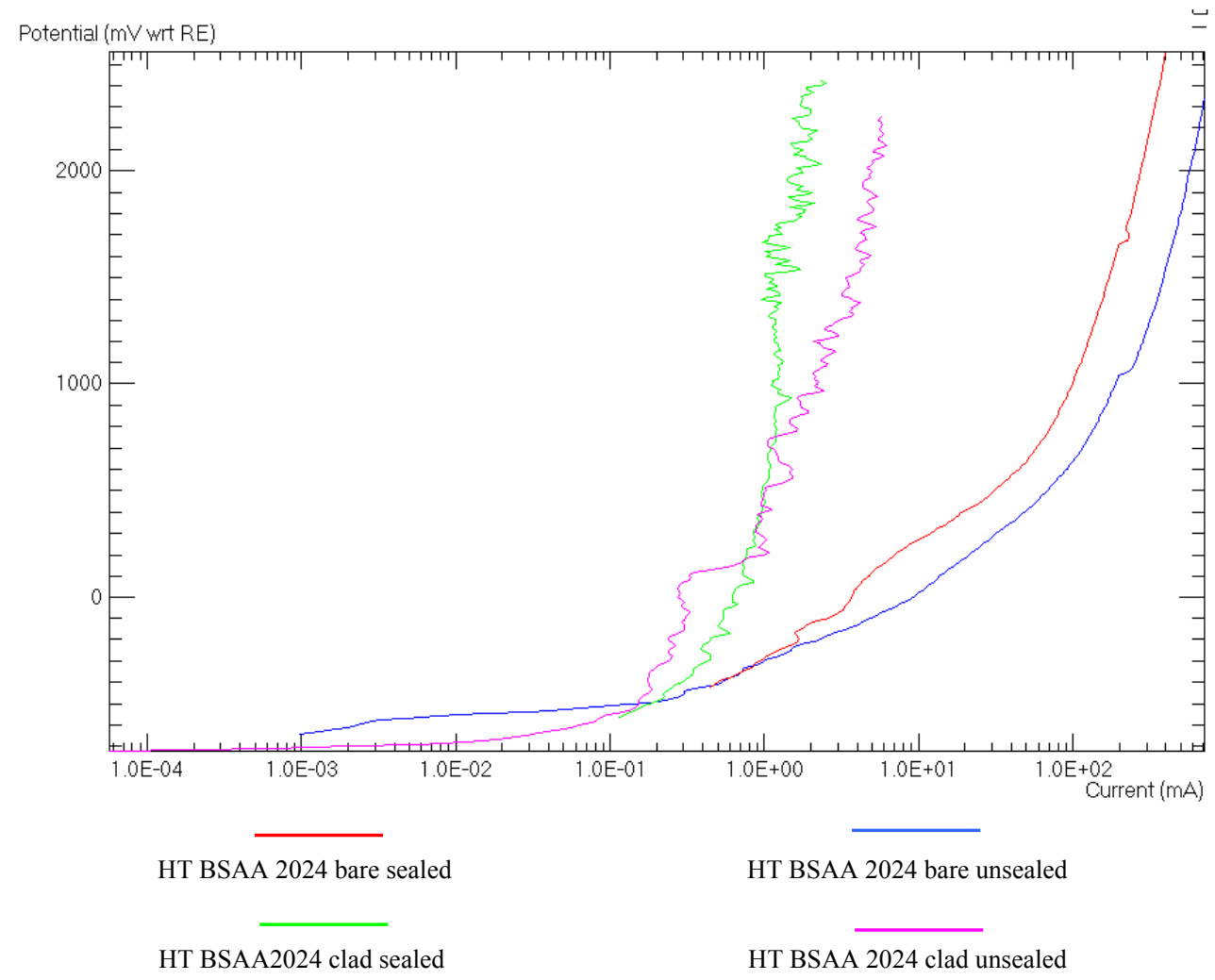

Figure 32 - Linear polarisation curves for HT BSAA processed 2024-T3 aluminium alloy

For the CAA processed 2024-T3 alloys, the influence of the cladding on the alloy affects the corrosion resistance to a lesser degree; Figure 33.

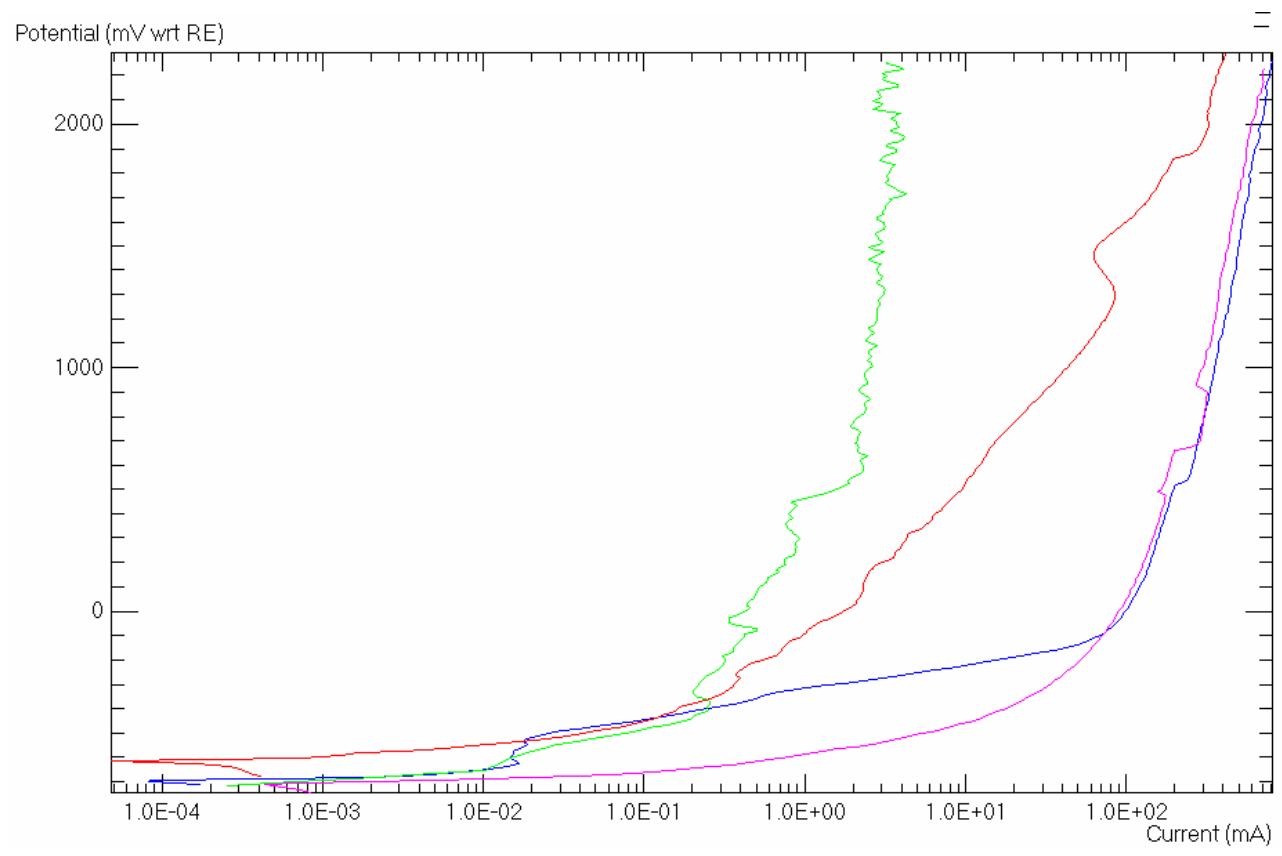


Figure 33 - Linear polarisation curves for CAA processed 2024-T3 aluminium alloy

From these data it is apparent that, both the HT BSAA and the CAA processed 2024T3 clad and bare alloys have very similar LP curves suggesting that the two processes have similar corrosion resisting properties. Marginal differences were indicated by the limiting current, which showed that the HTBSAA 2024-T3 bare alloy performed slightly better than the CAA treated material. With 2024-T3 clad material, the HT BSAA treated material surpassed the CAA.

The erratic nature of the current at higher potentials for all of these anodised surfaces is indicative of a thin oxide film protecting the various alloys but the film continually undergoing breakdown and repair during the polarization scan. This type of corrosion is most evident on the clad alloys, which may be explained as the corrosion caused by the increasing potential, penetrates the oxide layer which exposes the reactive pure aluminium underlying which then oxidises to form a protective oxide layer again. This process continuing until the cladding is fully oxidised. Recent electrical impedance spectroscopy (EIS) studies have demonstrated a high temperature sulphuric acid/boric acid/sodium borate electrolyte to produce corrosion resistant oxides comparable to that of CAA anodic oxide.

\subsubsection{Anodic coating weights}

As previously discussed, optimisation of the coating weight is important in order to maintain barrier corrosion resistance but to reduce bare metal fatigue loss. There are three simple ways of altering the coating weight for the HTBSAA process by varying the anodising voltage; anodising time, or; acid concentration in the electrolyte. For a constant voltage, the effect of increasing the anodising time causes an increase in film thickness until equilibrium is reached where dissolution rates are equivalent to formation rates and the oxide remains constant. Figure 34 illustrates the coating weight for the HTBSAA as a function of time. There is no apparent levelling of the slope with increasing time suggesting that the HTBSAA process can produce much thicker anodic oxides than used in the present study. Previously, it was shown that 
variations in sulphuric acid concentration within the electrolyte effects the film thickness. A reduction in sulphuric acid concentration was shown to reduce the film thickness.

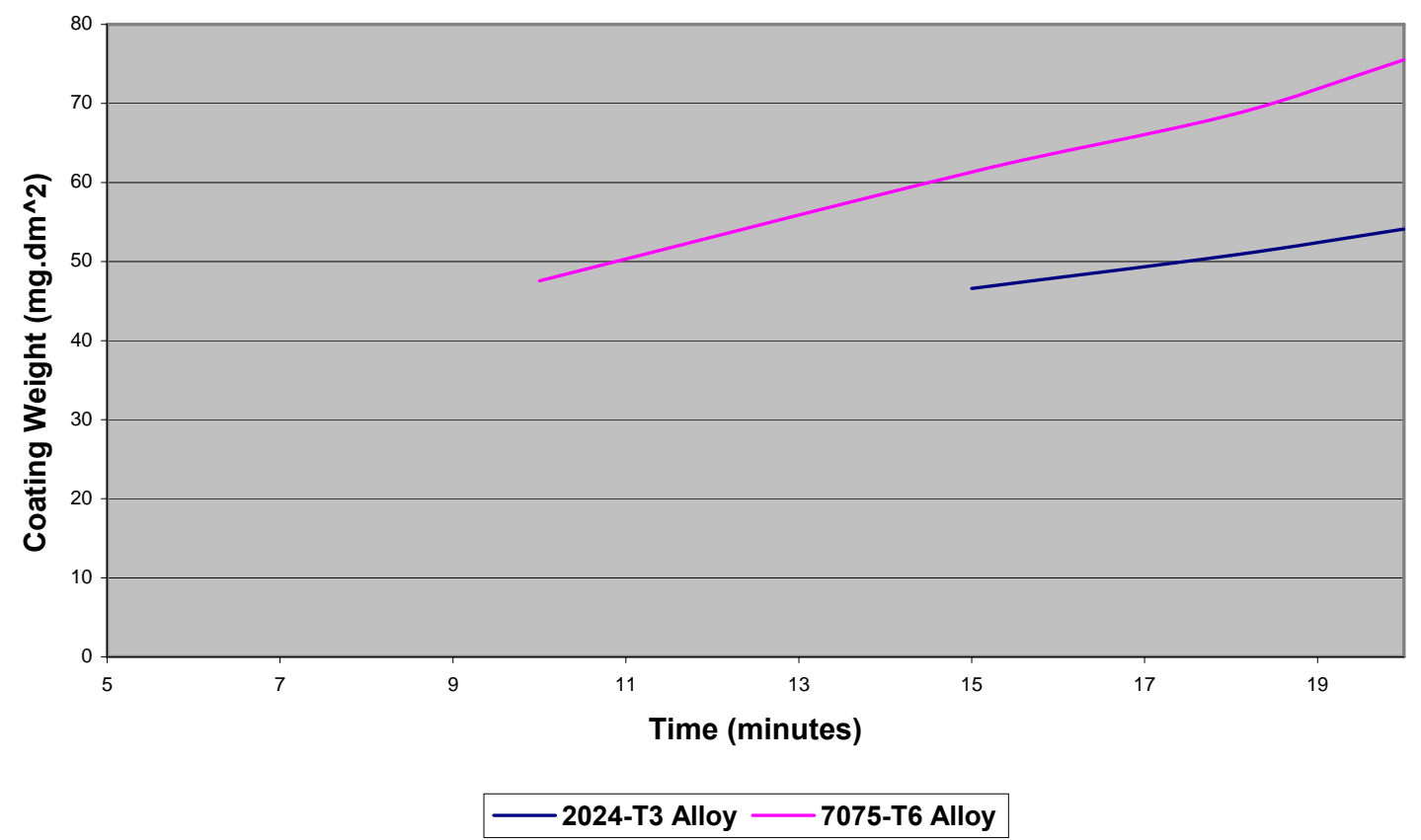

Figure 34 - Graph of coating weights for HT BSAA process as a function of time

If high coating weights were to be attributed to poor fatigue performance it is clear from Figure 34 that a reduced anodising time may solve this problem. A comparison of coating weights for the standard CAA and BSAA and HTBSAA processes are shown in Table 17 for the various alloys.

\begin{tabular}{|l|l|l|}
\hline Alloy Substrate & 2024-T3 bare & 2024-T3 clad \\
\hline CAA 40/50V & 48.4 & 70.0 \\
\hline Standard BSAA & 40.1 & 60.7 \\
\hline HT BSAA & 45.5 & 86.6 \\
\hline
\end{tabular}

Table 17 - Summary of coating weights for various processed alloy substrates 
As indicated in Section 1, the most successful metal bond pretreatments provide surfaces which have the following properties: freedom from contamination; wettability; macro- or micro-roughness; mechanical stability, and; hydrolytic stability. In this section the various treatments will be considered in terms of how they modify the surface in terms of these properties and, importantly, how the modified surfaces function in terms of adhesion performance and corrosion resistance.

\subsection{Degreasing}

The degreased-only surface was considered of interest since it is the minimum pretreatment which would, in practice, be carried out prior to bonding. The characterisation results obtained for the degreased-only 2024-T3 alloy are in good agreement with those reported in the literature. SEM showed that the surface topography of this alloy was relatively featureless with only shallow rolling lines and scratches visible. This observation was confirmed using stylus profilometry which gave $R_{a}$ and $R_{t}$ values close to $0.07 \times 10^{-6} \mathrm{~m}$ and $0.6 \times 10^{-6} \mathrm{~m}$ respectively.

The degreased-only aluminium alloy was shown, by AES, to possess levels of surface organic contamination consistent with monolayer or sub-monolayer coverage. The presence of organic contamination was confirmed by water contact angles, giving values between $40^{\circ}$ and $70^{\circ}$. A clean metal surface would be expected to have a free energy much greater than the $72.8 \mathrm{~mJ} . \mathrm{m}^{-2}$ required to enable the triply distilled water used to completely wet the surface. The presence of high levels of magnesium in the outermost atomic layer of the degreased-only bare alloy substrates was not unexpected and has previously been reported by Pocius ${ }^{111}$ and Kinloch and Smart ${ }^{120}$ amongst others. The role of the magnesium has been discussed by a number of workers. It is most likely that this provides a hydrolytically stable, but mechanically unstable or friable oxide. A number of studies have shown degreased-only 5000 series alloys to fail within this friable oxide layer. Furthermore, Pocius ${ }^{11}$ has shown that this oxide is removed by FPL etching, this may explain to some extent why an increase in adhesion is observed when using etched alloys for adhesive bonding. AES depth profiling indicated an oxide thickness of approximately $20 \mathrm{~nm}$ for the 2024-T3 clad alloy. 
These results demonstrate that degreasing serves only to remove gross organic contamination from the surface of the aluminium alloys and does not alter the surface structure or oxides present on the surface.

The degreased-only 2024-T3 clad alloy gave a mean initial SLS joint strength of $3350 \pm 200 \mathrm{~N}$. This compares very unfavourably with all of the chemical or electrochemical processes used. Visual inspection indicated apparent interfacial failure. Similarly poor joint test performance was observed in cyclic fatigue testing with degreased only 2024-T3 clad adherends failing in the dry ambient after a mean value of approximately 2200 cycles and 3400 in the wet. Again, these values were very low compared with other pretreatments. In addition, the polarisation results showed relatively poor corrosion resistance for the degreased-only alloy with a relatively high limiting current particularly in the anodic direction.

In summary, the degreased only surface provides few of the physicochemical properties, which would be expected to impart good adhesion. This has been confirmed by joint testing. In addition, the relatively poor corrosion resistance offered by this surface would be expected to compromise joint durability when exposed to hot wet environments for considerable periods of time.

\subsection{Alkaline cleaning}

With the aluminium alloy coupons supplied the standard Isoprep 44 process had little or no effect on topography compared with the degreased-only surface. This was concluded from both SEM and AFM data. Similarly, with 2024-T3 clad alloy AES showed little, if any, change in surface or sub-surface compositions following Isoprep 44 treatment with the introduction of only low levels of silicon on the alkaline cleaned surface. It should be noted that the coupons supplied had not been exposed to outdoor or non-optimised storage conditions and so were not excessively contaminated or hydrated. The alkaline clean procedure would be expected to be more beneficial to aluminium alloy sheets exposed to industrial environments. Since the deoxidised-only surface is rarely used as a stand alone prebond treatment for aluminium alloys the Isoprep 44 process was not evaluated in either joint or corrosion tests 


\subsection{Deoxidising}

\subsubsection{Standard deoxidisers}

The standard 'optimised' FPL and Tri-acid etched surfaces displayed virtually identical surface morphology and chemistry, both exhibiting a classical scalloped texture, with these features in the order 0.5 to $1 \mu \mathrm{m}$ across. In addition, there exists a reasonably similar oxide thickness of 20 to $40 \mathrm{~nm}$. Since these features are important in terms of increased surface roughness to aid adhesive bonding then it would be expected that both surface treatments would result in improved adhesive bonding performance compared with the degreased-only case. Such an increase is widely reported for the optimised FPL etch in the literature and has been attributed to the formation of a "microcomposite interphase". The removal of the previously described friable oxide layer would also be of benefit.

Both standard acid etches gave carbon levels at a maximum of $15 \%$ in AES, with water contact angles below $10^{\circ}$. These results indicate that a subsequently applied primer or adhesive would be able to intimately wet the FPL or Tri-acid surfaces to take advantage of the aforementioned topography.

From the data in Table 9, the obvious benefits to using acid etching as a pretreatment prior to bonding aluminium can be seen, if only for initial bond strengths. SLS values more than doubled for acid etched surfaces in comparison to the degreased-only joints with mean values close to $7300 \pm 200 \mathrm{~N}$ in both cases. Furthermore, a large increase in fatigue performance was observed in both wet and dry conditions using the FPL etch compared with the degreased-only adherends with up to $\sim 2.3 \times 10^{6}$ cycles-to-failure in the dry. The Tri-acid etch gave the expected improvement but did not perform as well as the FPL. The Tri-acid gave approximately $7.2 \times 10^{5}$ cycles-to-failure in dry conditions. Note that low levels of fluorine were observed on the Tri-acid etched surface, this has been noted by many workers to reduce the adhesion performance of aluminium pretreatments possibly by the formation of friable fluorine containing salts or by initiation of corrosion.

\subsubsection{Non-standard deoxidisers}


The case for investigating a suitable alternative chromate-free deoxidiser as a pretreatment prior to anodising is based on environmental and health and safety grounds. SEM showed both the Pyrene 10-21 and 14-19 processes to produce surfaces which were not uniformly deoxidised. Therefore, these processes were not studied further. If any benefits to adhesion are gained from the existence of etch pits, which other studies have suggested, then the alternative deoxidiser 14-73 has demonstrated similar surface structure and chemistry to that of the currently used FPL etch or Tri-acid etch and so was later evaluated in the standard BSAA process. In addition, the Chemcid 2218 process gave a micro-rough surface, albeit not scalloped, and so was also used in wedge testing with a subsequent BSAA treatment.

Both the Pyrene 14-73 and Chemcid 2218 combined with BSAA gave comparable or worse wedge test performance than the standard BSAA incorporating the Tri-acid etch. In both cases there was significant apparent interfacial failure when viewed under an optical microscope.

The usefulness of the EPAD process was initially demonstrated by the work of Tarr and Holmquest ${ }^{121}$. In contrast to the other deoxidisers studied, the EPAD process provides a highly nodular surface texture with features, typically $50 \mathrm{~nm}$ in diameter. Despite the observed beneficial surface morphology produced by the EPAD this is not a true deoxidising process, regardless of its reported etching capability. The work presented herein indicates that the EPAD produces a relatively thick oxide, in the range 200 to $300 \mathrm{~nm}$ thick, with a structure similar to an anodic oxide produced by PAA. This would explain the increased bond durability in wedge testing as the PAA oxide is well established as being capable of producing strong durable joints. Corrosion studies reported in the literature have shown the EPAD plus BSAA to provide hydration resistance equivalent to that of CAA, which a PAA oxide alone would not offer. This suggests that for the oxide film formed from the EPAD plus BSAA process, the EPAD surface topography is providing the necessary morphology for durable bonds to be formed. Either due to primer penetration of the nodular EPAD surface morphology on the uppermost section of the oxide film or as a result of the increased surface roughness on the nano-scale, which would provide greater surface area for the formation of chemical bonding. The EPAD plus BSAA process provides equivalent wedge test data to the standard CAA process with cohesive failure of the 
adhesive observed in all cases. Furthermore, the underlying BSAA oxide is possibly providing the corrosion resistance such that the duplex oxide is capable of offering the same bond durability and corrosion resistance to that of currently used CAA.

The usefulness of deoxidisers such as the FPL and Tri-acid etches as stand-alone pretreatments prior to structural adhesive bonding has been reported by many workers and further demonstrated in the present study. Both processes providing desirable surface properties, as discussed. However, it should be noted that in the present study the deoxidiser is considered primarily as a preliminary stage in the complete BSAA or CAA process. In this role, the deoxidiser is expected to provide an open surface structure with a relatively thin oxide, which would enable subsequent availability of aluminium cations for the anodic growth process. The aforementioned FPL and Triacid processes achieve this result. In contrast, the Pyrene 10-21 and 14-19 do not achieve this effect, providing non uniformly deoxidised surfaces. The Pyrene 14-73 and Chemcid 2218 processes provide promising open surface structures but do not improve upon the existing acid etches. The most interesting, nominal deoxidiser, is the EPAD process which provides the combined EPAD plus BSAA process with a highly desirable PAA-like structure in the outer region of a duplex oxide and with a more densely packed inner.

\subsection{Anodising}

In contrast to the degreased-only and to a lesser degree the acid etched surfaces, the lack of any residual organic material, as shown from XPS and AES data, after standard CAA and various BSAA-based treatments suggests that a high surface free energy exists. This was again confirmed with the use of contact angle analysis. All anodised surfaces having a zero or near zero degree water contact angle.

In the case of aluminium oxide the surface free energy has been reported to be up to $\sim 640 \mathrm{~mJ} . \mathrm{m}^{-2}{ }^{16}$. Again, the significance of this is that following both standard CAA and BSAA-based treatments an adhesive or primer would be expected to fully wet the surface. For a surface to be fully wetted by an amine cured epoxide a surface free energy of approximately $45 \mathrm{~mJ} . \mathrm{m}^{-2}$ or greater is required. 


\subsubsection{Standard CAA 40/50V Process}

When viewed on the macro-scale the standard CAA 40/50V anodised substrates, in plan view, appears to possess the scalloped texture found in the FPL etched surface. Other studies have clearly shown that both the micro- and macro-scale surface morphology produced by the deoxidising stage, transfers through on to the anodised surface. Such an effect was observed with all anodised surfaces in this study.

An additional effect associated with CAA processing of the 2024-T3 and 7075-T6 bare alloys are holes or voids present in the anodic oxide film itself, which were observed to be up to 5 to $10 \mu \mathrm{m}$ in diameter. This can be explained by the enhanced attack along grain boundaries and deep cavities on the surface, associated with the dissolution of second phase, intermetallic particles. 2214 alloys have shown the same cavities in anodic films, which were in the order of $\sim 20 \mu \mathrm{m}$, similar to the above observations and again this was explained by the dissolution of copper-rich intermetallic particles. Such voids were not observed on the clad anodised surfaces.

The majority of reported studies describe the cross-sectional structure of CAA oxide as a uniform and columnar. In the present study such idealised, regular hexagonal porous structures were only observed on clad alloys. The presence of the second phase particles including $\mathrm{Cu}-\mathrm{Al}$ within the 2024-T3 alloy is the reason for the nodular structure. Work using an Al-3.5\% Cu binary alloy has displayed the same nodular structure when anodised in sulphuric acid ${ }^{88}$.

What is clear is that the standard Bengough-Stuart CAA process provides a relatively open porous structure with pore diameters typically in the range 15 to $30 \mathrm{~nm}$. On the clad aluminium alloys the structure is relatively columnar but is branched, and contains significant voids on the bare alloy. Eddy current measurements and electron microscopy indicated that the CAA oxide thickness is typically in the range 2.5 to $3.5 \mu \mathrm{m}$. XRD data, not presented in this paper, indicated that the CAA oxide was amorphous. The chemistry of the anodic oxide has been reported as comprising hydrated alumina. This was confirmed in the present study using FTIR analysis. In the present study, FTIR showed the films to lose water at a temperature of greater than 
$100^{\circ} \mathrm{C}$, this could be significant if curing high temperature adhesives. Field emission gun SEM (FEGSEM) was used to show that the CAA oxide exhibited pore penetration by the primer to provide the expected good adhesion. Complete pore penetration, albeit of the much thinner PAA oxide has been reported by Arayasantiparb et al $^{122}$. This was confirmed using initial SLS tests. More significantly, SLS joints produced using 2024-T3 bare alloy and exposed to water at $60^{\circ} \mathrm{C}$ for 100 days had a residual joint strength of $\sim 60 \%$. This durability compares very favourably with other pretreatments reported in the literature. In addition, fatigue performance was much improved over the degreased-only case with the mean number of cycles-to-failure exceeding $3 \times 10^{6}$ in both dry and wet conditions. The extended oxide would be expected to provide excellent barrier corrosion resistance. This was confirmed using linear polarisation experiments, with a relatively low limiting current observed particularly in the anodic direction. This was particularly the case for the sealed CAA process. The usefulness of the sealed CAA for structural bonding has, however, not been demonstrated, it being more routinely used as a pre-paint process.

\subsubsection{Standard BSAA Process}

The Boeing BAC 5632 standard BSAA anodic film structure and chemistry was, as possibly expected, very similar to that reported to be produced by the SAA electrolyte. The oxide structure has finer pores, is more uniform in terms of coating, and non-branching in comparison to that of the CAA. Of interest, it should be noted that in the literature, SAA oxides produced on 2024 clad alloy have been demonstrated to exhibit some pore penetration with a phenolic resin ${ }^{123}$. It is this feature for the epoxy-phenolic primer system used in this study which is believed to be the key element affecting bond durability for the various modified BSAA processes. However, no studies have been identified which confirm the transport of epoxy-phenolic resins into the SAA pore structure. The pore diameter on the BSAA surface was estimated to be close to, or less than 10nm, much less than on the CAA. The thickness of these anodic oxides are, however, comparable being approximately 2.0 to $3.5 \mu \mathrm{m}$ depending on the alloy being processed.

Initial SLS results for the standard BSAA and CAA processes were comparable with values close to $8000 \pm 200 \mathrm{~N}$. However, the relevance of SLS testing must be considered in these cases. From all the data presented in this work, the SLS test 
appears only to be able to distinguish between poor (degreased or grit-blasted) joints and good (deoxidised or anodised) joints. The method fails to discriminate between different anodising parameters. It can be concluded that the SLS test method used was not selective enough to be able to distinguish between surfaces giving good and very good joint strengths. Further evidence for this is that all chemical and electrochemical treated surfaces displayed cohesive failure within the adhesive, with no other observed failure mechanisms. In this case, with high peel forces, the measurement is of the mechanical properties of the adhesive alone and not the interfacial adhesion. For this reason, the wedge test was used more extensively in the present study. Significantly, wedge test data confirms the superior bond performance offered by the CAA process compared with the standard BSAA.

It is highly likely that the primer/oxide interphase is of great importance in understanding this result. There was no evidence from FEGSEM to indicate any primer penetration into the $10 \mathrm{~nm}$ wide pores on the standard BSAA surface. Whereas, for the CAA with its pore diameters up to $30 \mathrm{~nm}$, the primer was demonstrated to penetrate to form a continuous interphase. Furthermore, the microand nano-rough surfaces produced by these surface treatments, if fully wetted, will both maximise the surface area available for primary and secondary bonding interactions. The role of such an interphase or nanocomposite can be postulated upon as photoelastic studies have shown stresses to be redistributed within the volume of adhesive rather than at a planar surface. FEA based techniques could, given sufficient computing power, be used to provide additional information in this area. What is clear is that some degree of pore penetration is required to produce optimum adhesion levels and that the standard BSAA process does not facilitate this. The failure analysis reported in Section 3.2.1 confirms this conclusion. Significantly, whilst the wedge test joints assembled with CAA treated adherends failed cohesively within the adhesive the standard BSAA treated joints demonstrated mixed mode failure. The barrier properties of the standard BSAA oxide would be expected to provide good corrosion resistance. Comparable, if not superior, corrosion resistance to the CAA process was observed in this study and is documented in the literature.

In summary, the standard BSAA provides a contamination-free, corrosion resistant surface, which does provide useful initial adhesion levels. However, wedge test 
results indicated that the lack of primer penetration and interphase formation compromises adhesion levels. Some degree of process optimisation is, therefore, required to create a different nanostructure capable of providing such an interphase.

\subsubsection{BSAA-based Processes}

The use of alternative alkaline cleaners and deoxidisers has been considered previously. This section will focus upon the use of different anodising parameters to achieve interphase formation namely; temperature, voltage, electrolyte concentrations and the use of a post anodising dip (PAD).

\subsubsection{The influence of varying electrolyte temperature}

BSAA deposition was carried out with electrolyte temperatures in the range of 15 to $40^{\circ} \mathrm{C}$. At $40^{\circ} \mathrm{C}$ visible "burning" of the aluminium alloy surfaces was evident and the anodic film was highly non-uniform in appearance. The optimum temperature which gave surface topography most similar to that produced by the CAA process, was $35^{\circ} \mathrm{C}$. This was defined as the high temperature BSAA (HTBSAA). All available characterisation techniques showed the HTBSAA to provide the same surface structure as the CAA in terms of both pore diameter and oxide thickness. Furthermore, AES and water contact angles showed this surface to be atomically clean with no carbon detectable. XPS, AES and FTIR showed the bulk chemistry to be similar with the exception that there are low levels of boron and sulphur, identified by XPS, in the BSAA oxide. FTIR confirmed the presence of sulphate species present with 2024-T3 clad alloy.

Initial SLS joint strengths were comparable with both CAA and HTBSAA treated adherends. More significantly, environmentally exposed 2024-T3 and 7075-T6 bare alloys also showed similar levels of residual joint strength with exposure time. In addition, the dry fatigue tested HT BSAA joints gave mean values over $3 \times 10^{6}$ cyclesto-failure. This is comparable to the CAA and an improvement over the standard BSAA process. The wet tested cyclic fatigue joints exhibited failure within the metal in most cases so the relatively low number of cycles-to-failure indicated in this case is misleading. 
Wedge test results for the HTBSAA process indicated comparable bond durability than that of the CAA.

For the HTBSAA, it is suspected that the observed open pore structure, which mimics that of the CAA, has aided primer penetration deep into the oxide.

It has been suggested in the literature that peel testing can be more sensitive than wedge testing for lifetime predictions. A positive result to come from peel testing of the HTBSAA processed material was the unexpectedly high values obtained, in comparison to those from CAA processed joints. The large increase in peel strength for the HTBSAA process using the 2024-T3 clad alloy is difficult to explain. However, such an increase in peel strength has previously been reported for the EPAD plus BSAA when compared to the CAA using 2024-T3 bare alloy.

Of additional interest are the results of nano-hardness testing, not amplified upon in this paper, which demonstrated that the HTBSAA process produces a relatively compliant oxide compared with the CAA but which has a high threshold below which no fracture occurs. These properties would be expected to give good stress transfer characteristics to the HTBSAA oxide. Such properties are again, difficult to quantify or indeed model.

The unsealed HTBSAA processed 2024-T3 bare alloy failed the 336 hours salt spray exposure test whereas the equivalent unsealed CAA alloy would be expected to pass. The salt spray results did prove, however, that the sealed BSAA oxides at elevated anodising temperatures can be adequately sealed to pass 1000 hours neutral salt spray testing. In normal design conditions any exposed surfaces would normally be sealed if not in a bonded state so the relevance of these results for the unsealed substrates is debatable. This is further seen in the filiform corrosion testing where the above trend for the unsealed HTBSAA is not present and instead the results are comparable or better than those from the CAA process. In addition, corrosion studies within the literature have shown, using EIS, good corrosion performance for oxides generated using a very similar high temperature boric/sulphuric acid bath. LP data presented in this study showed the HTBSAA alloys to perform similarly or better than CAA treated equivalents. 


\subsubsection{The influence of variations in anodising voltage}

For practical reasons the BSAA anodising voltage could only be varied between 15 and $25 \mathrm{~V}$ in the present study. In general terms, electron microscopy revealed that there was no significant difference compared with the standard BSAA in the oxide structure, within these limits, which would have been expected to contribute towards improved adhesion. Wedge test results showed values of 15, 20 and $25 \mathrm{~V}$ to produce only comparable levels of adhesion to the standard BSAA process.

\subsubsection{The influence of electrolyte concentrations}

The boric and sulphuric acid concentrations were varied from $2.5 \mathrm{~g} .1^{-1}$ to $10 \mathrm{~g} \cdot 1^{-1}$ and $20 \mathrm{~g} .1^{-1}$ to $60 \mathrm{~g} .1^{-1}$ respectively. SLS testing showed all combinations to give comparable values, close to $8000 \pm 200 \mathrm{~N}$, to the standard BSAA incorporating $7.5 \mathrm{~g} .1^{-1}$ and $40 \mathrm{~g} . \mathrm{l}^{-1}$. The structure of the anodic oxides were similar in terms of their pore diameters, being close to 10 to $15 \mathrm{~nm}$ across, comparable to the standard BSAA.

The only significant difference that was noted for electrolyte concentration variations was that the oxide increased in thickness with increasing sulphuric acid content. This is attributed to the availability of more sulphuric acid with increased oxidising power at higher concentrations. Although this result does not impact upon this study it could have an influence in terms of the development of more corrosion resistant coatings for example in pre-paint applications based on the BSAA process.

\subsubsection{The influence of a post anodising dip (PAD)}

In terms of the PAD as a post treatment to the standard BSAA process, many of the reported studies have only discussed the use of a PAD on the anodised surface for time periods ranging from 10 to 240 seconds. In this study a 10 minute immersion in the PAD solution was studied. SEM showed the standard BSAA following the PAD to produce an open oxide network. This open structure would be expected to provide an interphase with the subsequently applied BR-127 primer. Note that large areas of this surface had much thinner oxide layer than the 2.5 to $3.5 \mu \mathrm{m}$ expected on the standard BSAA processed surface. It is possible that this extended time may have compromised the oxide barrier protection and so reduced the corrosion performance of the anodised surface. In simple terms this illustrates the conflicting requirements of a structural prebond process for metal bonding in that an open structure is required 
to permit primer or adhesive penetration but such a structure will be less corrosion resistant than a more compact oxide.

At this stage it is also worth noting a few general points on the relationship between surface topography and structural adhesion performance. From this work it would appear that the critical surface morphology necessary to provide good mechanical interlocking and stress transfer, in order to gain good bond strength durability, needs only to be within 200 to $300 \mathrm{~nm}$ of the top surface. It is unclear at this point how much further any primer or adhesive may penetrate the oxide and what, if any, influence this would have on durability. Work by Packham suggests that an epoxide will only penetrate a few hundred nanometres into a porous alumina structure which is only a few tens of nanometres in diameter. What is clear is that the uppermost surface must fully wet to allow penetration of the oxide. The viscosity of the primer or adhesive will play a large role in the final outcome. This has also been demonstrated within the literature whereby the oxide stability after treatment and the effects of wiping the surface, when using a primer in combination with the adhesive has shown little difference in bond durability, but a large difference has been revealed if the primer is omitted, giving evidence that the low molecular weight of the primer helps the penetration of the oxide surfaces. In the case of a porous morphology it is also evident that a critical pore diameter exists which will permit primer penetration. This is likely to be related to capillary forces of the primer trying to penetrate the pores and also the size of the molecules trying to migrate down into the oxide. The remaining oxide below this 'active' layer will contribute to the corrosion resistance of the surface treatment. This theory ties in with the joint performance of PAA processed material, which is known to provide excellent bond strength, attributed to the surface morphology of the thin oxide but lacks the corrosion performance offered by that of CAA or BSAA processed material, owing to a lack of the aforementioned thick additional oxide. Corrosion test data from neutral salt spray and full immersion plus LP from the present authors not reported here clearly demonstrate the improved passivation offered by the CAA process.

The BSAA plus PAD process produced good initial bond strength and durability. In particular, excellent adhesion performance was demonstrated in both wedge and fatigue tests. 


\section{CONCLUSIONS}

From Section 1 it is clear that an array of surface pretreatments are currently used for the structural bonding of aluminium and its alloys. For a particular alloy, these pretreatments can produce significant differences in terms of surface chemistry, morphology and cross-sectional structure. These changes occur on a macro-, microand nano-scale as evinced by techniques such as XPS, AES and electron microscopy. These techniques have proved of use in the present study.

In previous studies, different pretreatments applied to bonded joints or assemblies have revealed significant differences in terms their performance in either initial adhesion tests or durability. However, from both the available literature and the present study, it is apparent that the anodic oxides are the best performing pretreatments for aluminium alloys. In particular, in cases where joints would be exposed to hot-wet conditions the chromic acid anodise (CAA) has proven to be highly successful. The standard CAA $40 / 50 \mathrm{~V}$ process is routinely used in the highly demanding aerospace industry where absolute performance and safety are critical factors. There are, however, issues relating to the use of hexavalent chromium-based processes, such as the CAA, from both environmental and personal exposure points of view. There is, therefore, a current requirement to replace the standard CAA as a prebond process.

The success of the CAA process is due to the physicochemical properties imparted to the aluminium surface. The high energy, easily wettable, nano-rough, porous structure provides the opportunity for interphase formation and stress redistribution within the bondline, as discussed by Packham ${ }^{32}$, Rider and Arnott ${ }^{124}$ and others. The relatively thick, amorphous oxide provides barrier corrosion protection. Any replacement technology would be required to fulfil these roles to provide the required initial adhesion, long-term bond durability and corrosion resistance. It would appear from the present study, that although in some tests acceptable levels of adhesion can be achieved with basic cleaning and minimal surface pretreatments (degreased and deoxidised), the major contributing factor to achieving optimised adhesion is the 
nanometre-scale surface morphology and the ability of a primer to penetrate this morphology. The thick oxide is thereafter responsible for the durability by providing hydration or corrosion resistance.

The existing Boeing BAC 5632 boric sulphuric acid anodising (BSAA) process was expected to provide good initial adhesion and corrosion resistance due to its high energy surface and potentially thick oxide structure which is comparable to the sulphuric acid based process used for architectural purposes. For this reason it was thought worthy of study. However, the lack of available porosity on the standard BSAA surface meant that interphase formation was not possible with the epoxyphenolic primer used and wedge test results were not satisfactory, with mixed mode failure resulting. This result was of interest as it demonstrates that the macro-rough surface morphology, and any mechanical interlocking associated with it, has limited effect on bond durability.

A number of modifications were considered in this study to the standard BSAA process specifically to achieve satisfactory structural bond performance. These included: variations in the deoxidiser and anodising parameters, and; the use of a post anodising dip. It has been demonstrated in these studies that there are three possible methods of providing excellent durability using a variation of the standard BSAA process: the use of an electrolytic phosphoric acid deoxidiser (EPAD); a high temperature anodise at $35^{\circ} \mathrm{C}$, and; the use of a post anodise phosphoric acid dip (PAD). In all cases environmentally-aged wedge test results were comparable to the CAA control.

The EPAD plus BSAA process provides an outer layer, extending to a few hundred nanometres, of highly open porous oxide on top of a virtually non-porous BSAA structure. The open structure is initially deposited in the deoxidising stage with the anodic oxide subsequently grown underneath. The open porosity providing the possibility of the desirable interphase formation and the underlying oxide the corrosion protection. The outermost structure is comparable to the highly successful PAA process ${ }^{125}$ currently used for structural adhesive bonding. 
The HTBSAA process provides an hydrated oxide directly comparable in structure to the standard CAA and comprising only limited changes in the oxide chemistry. At high temperatures there is increased dissolution of the anodic film making the HTBSAA structure more open than the lower temperature standard process. The HTBSAA process can be used for structural adhesive bonding with equivalent if not superior adhesion performance to that of the CAA treatment. In addition, the corrosion protection offered by both processes are similar and the metal fatigue loss following HTBSAA is slightly less than that following CAA. As such, the HTBSAA process could be considered as a suitable drop-in replacement to the currently used hexavalent chromium processes operated in the aerospace industry today. In addition, the processing parameters can be engineered to provide a treatment suitable for both paint applications and corrosion protection as well as for the structural adhesive bonding of aluminium alloys.

The PAD process is proposed to operate by removal of the thinner pore walls in the standard BSAA film to produce a relatively open structure. This "opened-up" structure provides the possibility of interphase formation resulting in equivalent joint test results to the standard CAA process. Corrosion performance was not studied for this process but it would not be expected to perform to the same standard as the EPAD plus BSAA or HTBSAA due to the rather non-uniform oxide structure produced.

\section{REFERENCES}

1. Minford, J.D., Handbook of Aluminum Bonding Technology And Data, Marcel Dekker, New York, 1993.

2. Thrall, E.W. and Shannon, R.W., Adhesive Bonding of Aluminium Alloys, Marcel Dekker, New York, 1985.

3. MMillan, J.C., “Bonded Joints And Preparation For Bonding”, NATO Agard Lecture Series 102, 1979.

4. Brewis, D.M. in Durability of Structural Adhesives, Ed. Kinloch, A.J., Applied Science, London, 1983.

5. Sargent, J.P., Int.J.Adhesion \& Adhesives, 2005, 25, 247. 
6. Böhm, S., Dilger, K., Wisner, G. and Welters, T., Proc.SAEVII, Bristol UK, 2004, 184.

7. Dunkerton, S.B. and Vlattas, C., J.Materials \& Product Technology, 1998, 13(1,2), 105.

8. Kinloch, A.J. in Adhesion And Adhesives: Science And Technology, Chapman and Hall, London, 1987.

9. Liebrecht, F., Kleinert, H. and Kalich, J., Proc.SAEVII, Bristol UK, 2004, 126.

10. Haraga, K., Taguchi, K., Yoda, K. and Nakashima, Y., Int.J.Adhesion \& Adhesives, 2003, 23(5), 371.

11. Darwish, S.M, Int.J.Adhesion \& Adhesives, 2003, 23(3), 169.

12. Polmear, I.J., "Light Alloys Metallurgy Of The Light Metals”, $3^{\text {rd }}$ Ed, Arnold Press, London, 1995.

13. Dunkerton, S.B. and Vlattas, C., J.Materials \& Product Technology, 1998, 13(1,2), 105 .

14. Lumsden, B., Mahoney, M.W., Pollock, G. and Rhodes, C.G., Corrosion, 1999, 5(12), 1127.

15. Kinloch, A.J. “Adhesives In Engineering”, Proc.Inst.Mech.Eng., Part G, J.Aerospace Engineering, 1996, 211, 307.

16. Comyn, J., Adhesion Science, RSC Paperbacks, Cambridge, 1997.

17. Lee, L-H., Fundamentals of Adhesion, Plenum Press, New York, 1991.

18. Pizzi, A. and Mittal, K.L., Handbook Of Adhesive Technology, Marcel Dekker, New York, 1994.

19.Korenberg, C.F., Kinloch, A.J. and Watts, J.Adhesion, 2004, 80, 201.

20. Weitzenböck, J.R., Niese, F. and Hübschen, G., Proc.Adhesion'99, Cambridge, UK, 1999, 93.

21. Critchlow, G.W. and Brewis, D.M., Int.J.Adhesion \& Adhesives, 1996, 16, 255.

22. Rance, D.G, in Industrial Adhesion Problems, Ed.D.M.Brewis, Orbital Press, Oxford, 1985.

23. Synytska, A. et al, J.Adhesion, 80(8), 2004, 667.

24. Minford, J.D., J.Appl.Polymer Sci., 1977, 32, 91.

25. Minford, J.D., SAMPE Quarterly, 1978, 9, 18.

26. Hong, S.G, and Boerio, F.J., J.Adhesion, 1995, 49, 133.

27. Olsson-Jacques, C.L., Wilson, A.R., Rider, A.N. and Arnott, D.R., Surface \& Interface Analysis, 1996, 24, 569. 
28. P.W.Webb, PhD Thesis, Loughborough University, UK, 2005.

29. Chen, J.M., Sun, T.S. and Venables, J.D., SAMPE J., 1978, 22.

30. G.W.Critchlow and D.M.Brewis, Int.J.Adhesion \& Adhesives, 1995, 15, 173.

31. Rider, A.N and Arnott, D.R, Proc.Euradh'98/WCARP, Garmisch-Partenkirchen Germany, 1998.

32. D.E.Packham in Proc.First Int.Congress on Adhesion Science and Technology, Eds. Van Ooij, W.J. and Anderson, H.R., Amsterdam Holland, 81.

33. Poole, P. and Watts, J.F., Int.J.Adhesion \& Adhesives, 1985, 5(1), 33.

34. Maddison, A. and Critchlow, G.W., J.Adhesion, 1996, 55, 273.

35. Safavi-Ardebili, V., Sinclait, A.N. and Spelt, J.K., J.Adhesion, 1997, 62, 93.

36. Roche, A.A., Bouchet, J. and Bentadjine, S., Int.J.Adhesion \& Adhesives, 2002, 22(6), 431.

37. Dillingham, R.G. and Boerio, F.J., J.Adhesion, 1987, 24(2-4), 315.

38. Bockenheimer, C., Valeske, B. and Possart, W., Int.J.Adhesion \& Adhesives, 2002, 22(5), 349.

39. Brockmann, W. , Hennemann, O.D. and Kollek, H., Int.J. Adhesion \& Adhesives, $1982,6,33$.

40. Davis, G.D., Sun, T.S., Ahearn, J.S. and Venables, J.D., J.Materials Science, 1982, 17, 1807.

41. Venables, J.D., J.Materials Science, 1984, 19, 2431.

42. Kinloch, A.J., Proc.Inst.Mech.Eng., 1996, Preprint No.8.

43. Arnott, D.R and Kindermann, M.R., J.Adhesion, 1995, 48, 101.

44. Bishopp, J.A., Sim, E.K., Thompson, G.E. and Wood, G.C., J.Adhesion, 1988, 26, 237.

45. Lu, Y.-F. and Aoyagi, Y., Jpn.J.Appl.Phys., 1994, 33, 430.

46. Critchlow, G.W., Cottam, C.A., Brewis, D.M and Emmony, D.C., Int.J.Adhesion \& Adhesives, 1997, 17, 143.

47. Abel. M-L, Digby, R.P., Fletcher, I.W. and Watts, J.F., Surface \& Interface Analysis, 2000, 29, 115.

48. Hobbs, P.M. and Kinloch, A.J., J.Adhesion, 1998, 66, 203.

49. Rider, A.N. \& Arnott, D.R., Int.J. Adhesion \& Adhesives, 2000, 20(3), 209.

50. Crook, R.A., Sinclair, J.W., Poulter, L.W. and Schulte, K.J., J.Adhesion, 1998, 68(3-4), 315. 
51. Taylor, C.E., Boerio, F.J., Ward, S.M., Ondrus, D.J., Dickie, R.A. and Brutto, M.M., J.Adhesion, 1999, 69(3-4), 237.

52. King, R.G., Surface Treatment And Finishing of Aluminium, Pergamon Press, Oxford, 1988.

53. Sheasby, P.G. and Pinner, R., The surface Treatment \& Finishing of Aluminium \& Its Alloys $6^{\text {th }}$ Ed. Vols $1 \&$ 2, Finishing Publications Ltd, 2001.

54. Digby, R.P. and Packham, D.E., Int.J.Adhesion \& Adhesives, 1995, 2, 61.

55. Schwartz, H., J.Appl.Polymer Sci: Appl.Polymer Symp., 1977, 32, 65.

56. Oosting, R., Proc.European Adhesion Conf., 1992, 635.

57. Hughes, A.E., Nelson, K.J.H. and Miller, P.R., J.Materials Science \& Technology, $1999,15,1124$.

58. Dickie, R., Haack, L.P., Jethwa, J.K., Kinloch, A.J. and Watts, J.F., J.Adhesion, $1998,66,1$.

59. Tarr, L.E. and Holmquist, H.H., Proc. $33^{\text {rd }}$ Int.SAMPE Symposium, 19902102.

60. Critchlow, G.W. and Brewis, D.M., J.Adhesion, 1997, 61, 213.

61. Cohen, S.M., Corrosion, 1995, 51(1), 71.

62. Briskham, P. and Smith, G., Int.J.Adhesion \& Adhesives, 1999, 20, 33.

63. Underhill, P.R., Rider, A.N. and Duquesnay, D.L., Int.J.Adhesion \& Adhesives, 2003, 4, 307.

64. Lunder,O, Lapique, F., Johnsen, B. and Nisancioglu, K., Int.J.Adhesion \& Adhesives, 2004, 24, 107.

65. Maddison, A. and Critchlow, G.W., J.Adhesion, 1996, 55, 273.

66. Marwick, W.F and Sheasby, P.G., SAE Tech.Paper 870151, 1987.

67. Thompson, P.J. and Heaton, H.B., Trans.Inst.Metal Finishing, 1980, 58, 81.

68. Minford, J.D, Adhesives Age, 1980, 36.

69. Bjorgum,A., Lapique, F., Walmsley, J. and Redford, K., Int.J.Adhesion \& Adhesives, 2003, 23, 401.

70. Hadavinia, H., Kinloch, A.J, Little, M.S.G. and Taylor, A.C., Int.J.Adhesion \& Adhesives, 2003, 23, 449.

71. Arrowsmith, D.J. and Maddison, A., Int.J.Adhesion \& Adhesives, 1987, 7(1), 15.

72. Vine, K, Cawley, P. and Kinloch, A.J., J.Adhesion, 2001, 77, 161.

73. Digby, R.P. and Packham, D.E., Int.J. Adhesion \& Adhesives, 1995, 15, 61.

74. Yendall, K.A., PhD Thesis, Loughborough University, 2003

75. Rostami, S.D. and Kellar, E.J.C., Proc.SAEVII, Bristol UK, 13. 
76. Lunder, O., Olsen, B. and Nisancioglu, K., Int.J.Adhesion \& Adhesives, 2002, 22, 143.

77. Cohen, S.M. and Spadafora, S.J, Naval Air Warfare Center Report .

NADCADWAR-95023-43,1995.

78. Sykes, J., Thompson, G.E., Mayo, D. and Skeldon, P., J. Materials Science, 1997, $32,4909$.

79.Thompson, G.E., Habazaki, H., Shimizu, K., Sakairi, M., Skeldon, P., Zhou, X., and Wood, G.C., Int.J.Aircraft Engineering \& Aerospace Technology, 1999, 71 (3), 228.

80. Jessensky, O., Müller, F. and Gösele, U., J.Electrochem.Soc., 1998, 145(11)

81. Li, A.P., Müller, F., Birner, A., Nielsch, K. and Gösele, U., J.Applied Physics, 1998, 84(11), 6023.

82. Shawaqfeh, A.T. and Baltus, R.E., J.Electrochem.Soc., 1998, 145(8), 2699.

83. Li, F., Zhang, L. and Metzger, R.M., Chemical Materials, 1998, 10, 2470.

84. Graeve, D.E., Terryn, H and Thompson, G.E., J,Appl.Electrochem., 2002, 33, 73.

85. Dimogerontakis, T., Kompotiatis, L. and Kaplanoglou, I., Corrosion Science, 1998, 40(11), 1939.

86. Skeldon, P., Zhou, X., Thompson, G.E., Wood, G.C., Habazaki, H. and Shimizu, K., Corrosion Science, 1999, 55(6), 561.

87. Kihn, Y., Thompson, G.E., Galaup, G., Skeldon, P., Zhou, X., Shimizu, K. and Habazaki, H., Corrosion Science, 2000, 42, 533.

88. Paez, M.A., Bustos, O., Thompson, G.E., Skeldon, P., Shimizu, K. and Wood, G.C., J.Electrochem.Soc., 2000, 147(3), 1015.

89. Liu, Y., Skeldon, P., Thompson, G.E., Zhou, X., Habazaki, H. and Shimizu, K., Corrosion Science, 43, 2349.

90. Bonilla, F.A., Berkani, A., Skeldon, P., Thompson, G.E., Habazaki, H., Shimizu, K., John, C. and Stevens, K. , Corrosion Science, 2002, 44, 1941.

91. Zhou, X., Thompson, G.E., Habazaki, H., Paez, M.A., Shimizu, K., Skeldon, P. and Wood, H., J.Electrochem.Soc., 2000, 147(5), 1747.

92. Liu, Y., Skeldon, P., Thompson, G.E., Habazaki, H. and Shimizu, K., Corrosion Science, 2002, 44, 1133.

93. Domingues, L., Fernandes, J.C.S., Da Cunha Belo, M., Ferreira, M.G.S. and Guerra-Rosa, L., Corrosion Science, 2003, 45, 149.

94. Boeing Process Spec.BAC 5632. 
95. Leland, T. and Moji, Y., Proc. $26^{\text {th }}$ Annual Aerospace/Airline Plating \& Metal Finishing Forum \& Exposition, 1990, 1-6.

96. "Boric/Sulfuric Acid Anodising and Thin Film Sulfuric Acid Anodising - An in House Exaluation by METALAST International, Inc.”, Metalast Technical Bulletin, 2000, 1-5.

97. Cree, A.M. \& Weidmann, G.W., Trans.Inst.Metal Finishing, 1997, 75(5), 199.

98. Mnich, J., Proc. $1^{\text {st }}$ Int.SAMPE Environmental Conference, 1991, 371.

99. Mnich, J. and Schoneman, C., Proc.38 ${ }^{\text {th }}$ Int.SAMPE Symp., 1993, 819.

100 .Mnich, J., Proc.39 ${ }^{\text {th }}$ Int.SAMPE Symp., 1994, 2771.

101. SIFCO Selective Plating UK Ltd, Product Finishing, 1995, 48, 26.

102. Arrowsmith, D.J. and Clifford, A.W., Int.J.Adhesion \& Adhesives, 1985, 5(1), 40.

103. Pike, R.A, Patarini, V.M, Zatorski, R. and Lamm, F.P., Int.J.Adhesion \& Adhesives, 1992, 12(4), 227.

104. Dillard, J.G, Wolfe, K.L. and Holmes, B.L., J.Adhesion, 1997, 62(1-4), 113.

105. Davis, G.D., Groff, G.B. and Zatorski, R.A., Surface \& Interface Analysis, 1997, 25,366 .

106. Gendler, Z., Rosen, A., Bamberger, M., Rotel, M, Zahavi, J, Buchman, A. and Dodiuk, H, J.Materials Sci., 1994, 29, 1521.

107. Broad, R., French, J. and Sauer, J., Int.J.Adhesion \& Adhesives, 1999, 19, 193.

108. Sancaktar, E., Babu, S.V., Zhang, E., D’Couto, G.C. and Lipshitz, H., J.Adhesion, 1995, 50, 103.

109. Elbing, F., Anagreh. N., Dorn, L. and Uhlmann, E., Int.J.Adhesion \& Adhesives, 2003, 23(1), 69.

110. D.M.Brewis, G.W.Critchlow and C.A.Curtis, ., Int.J.Adhesion \& Adhesives, $1999,19,253$.

111. Pocius, A.V., J.Adhesion, 1992, 39, 101.

112. Mol, J.M.C., Hinton, B.R.W., Van der Weijde, D.H., de Wit, J.H.W. and Van der Zwaag, S., J.Materials Science, 2000, 35, 1629.

113. Spathis, P. Poulis, J. and Papastergiadis, E., British Corrosion J., 1995, 30(3), 233.

114. Dasquet, J.-P., Bonino, J.-P., Caillard, D. and Bes, R., Proc.Surface Treatment IV, Southampton UK, 1999, 149. 
115. Chromic acid anodising of aluminium and aluminium alloys, MoD (UK) DEF STAN 03-24/3 (1996).

116. Standard test method for adhesive-bonded surface durability of aluminium (wedge test), ASTM D 3762-79.

117. Method for measurement of mass of coating on anodically coated aluminum, ASTM B137-95.

118. Standard practice for operating salt spray (fog) apparatus, ASTM B 117-97.

119. Paints and varnishes - determination of resistance to filiform corrosion, BS EN ISO 4632.

120. Kinloch. A.J. and Smart, N.R., J.Adhesion, J.Adhesion, 1981, 12(1), 1.

121. Tarr, L.E. and Holmquist, H.H., 35 ${ }^{\text {th }}$ Int.SAMPE Symp., 1990, 2102.

122. Arayasantiparb, D., McKnight, S. and Libera, M., J.Adhesion, 2001, 76, 353.

123. Hennemann, O.D. and Brockmann, W., J.Adhesion, 1981, 12, 297.

124. Rider, A.N., and Arnott, D.R., J.Adhesion, 2001, 75, 203.

125. Boeing Process Spec., Phosphoric Acid Anodising, BAC 5555 